\title{
NONDEGENERATE COHOMOLOGY PAIRING FOR TRANSITIVE LIE ALGEBROIDS, CHARACTERIZATION
}

\author{
JAN KUBARSKI AND ALEXANDR MISHCHENKO
}

\begin{abstract}
The Evens-Lu-Weinstein representation $\left(Q_{A}, D\right)$ for a Lie algebroid $A$ on a manifold $M$ is studied in the transitive case. To consider at the same time non-oriented manifolds as well, this representation is slightly modified to $\left(Q_{A}^{o r}, D^{o r}\right)$ by tensoring by orientation flat line bundle, $Q_{A}^{o r}=$ $Q_{A} \otimes \operatorname{or}(M)$ and $D^{o r}=D \otimes \partial_{A}^{o r}$. It is shown that the induced cohomology pairing is nondegenerate and that the representation $\left(Q_{A}^{o r}, D^{\text {or }}\right)$ is the unique (up to isomorphy) line representation for which the top group of compactly supported cohomology is nontrivial. In the case of trivial Lie algebroid $A=T M$ the theorem reduce to the following: the orientation flat bundle (or $(M), \partial^{o r}$ ) is the unique (up to isomorphy) flat line bundle $(\xi, \nabla)$ for which the twisted de Rham complex of compactly supported differential forms on $M$ with values in $\xi$ possesses the nontrivial cohomology group in the top dimension. Finally it is obtained the characterization of transitive Lie algebroids for which the Lie algebroid cohomology with trivial coefficients (or with coefficients in the orientation flat line bundle) gives Poincaré duality. In proofs of these theorems for Lie algebroids it is used the Hochschild-Serre spectral sequence and it is shown the general fact concerning pairings between graded filtered differential $\mathbb{R}$-vector spaces: assuming that the second terms live in the finite rectangular, nondegeneration of the pairing for the second terms (which can be infinite dimensional) implies the same for cohomology spaces.
\end{abstract}

\section{INTRODUCTION}

The cohomology pairing coming from Evens-Lu-Weinstein representation of a Lie algebroid [E-L-W] is very important in many applications of Lie algebroids (Poisson geometry, intrinsic characteristic classes). This pairing generalizes the well known pairings that give Poincaré duality for Lie algebra cohomology and de Rham cohomology of a manifold and real cohomology of transitive invariantly oriented Lie algebroids [K3]. For a Poisson manifold, this pairing agree with the pairing on the Poisson homology. The authors of [E-L-W] give an example of a nontransitive Lie algebroid for which the pairing is not necessarily nondegenerate and post the problem of when it is nondegenerate. This paper gives the positive answer for the case of any transitive Lie algebroids and proves the property of this representation: it is the one (up to isomorphy) for which the top group of compactly supported cohomology is nontrivial.

2000 Mathematics Subject Classification. Primary 58 H 99; Secondary 17 B 56, 18 G 40, 55 R 20, 55 T 05, 57 R 19, 57 R 22, 58 A 10.

Key words and phrases. twisted cohomology, cohomology of Lie algebras, Poincaré duality, Lie algebroid, modular class, cohomology pairing, Evens-Lu-Weinstein pairing, Hochschild-Serre spectral sequence.

This paper is in final form and no version of it will be submitted for publication elsewhere. 
Finally, we prove that for the nonregular transformation Lie algebroid corresponding to the action $\gamma: \mathbb{R} \rightarrow \mathfrak{X}(\mathbb{R}), \gamma(t)=t \cdot X$ where $X=x^{N} \frac{d}{d x}$, there is no line representation for which the cohomological pairing is nondegenerate.

More detailed, this paper is devoted to prove two cycles of theorems, mutually overcoming.

FIRST CYCLE concerns nondegenerate cohomology pairings for manifolds (Theorem 2.3), Lie algebras (Theorem 3.4) and Lie algebroids (Theorem 7.3).

- Assume that $M$ is a connected $m$-dimensional manifold (oriented or not) and $\xi_{1}, \xi_{2}$ are two flat vector bundles with flat covariant derivatives $\nabla_{1}$ and $\nabla_{2}$ respectively. Denote by or $(M)$ the orientation bundle with canonical flat structure $\partial^{\text {or }}$. Let $F: \xi_{1} \times \xi_{2} \rightarrow$ or $(M)$ be a pairing (i.e. 2-linear homomorphism) of vector bundles compatible with the flat structures $\left(\nabla_{1}, \nabla_{2}, \partial^{\text {or }}\right)$, nondegenerate at least at one point (therefore, at every). From such a pairing one obtains a pairing on differential forms and the induced pairing in cohomology

$$
H_{\nabla_{1}}^{j}\left(M, \xi_{1}\right) \times H_{\nabla_{2}, c}^{m-j}\left(M, \xi_{2}\right) \stackrel{F_{\#}}{\longrightarrow} H_{\partial^{\text {or }, c}}^{m}(M, \text { or }(M)) \stackrel{\int_{M}^{o r, \#}}{\longrightarrow} \mathbb{R}
$$

is nondegenerate in the sense that

$$
H_{\nabla_{1}}^{j}\left(M, \xi_{1}\right) \stackrel{\cong}{\longrightarrow}\left(H_{\nabla_{2}, c}^{m-j}\left(M, \xi_{2}\right)\right)^{*} .
$$

The index " $c$ " means that the compactly supported cohomology are considered. This theorem generalizes the classical Poincaré duality as well as the one for $d_{\omega^{-}}$ cohomologies [G-L].

- Assume that $\mathfrak{g}$ is an arbitrary $n$-dimensional Lie algebra and $\nabla_{1}, \nabla_{2}: \mathfrak{g} \rightarrow L_{\mathbb{R}}$ are two representations of $\mathfrak{g}$ in $\mathbb{R}$. Denote by $\nabla_{\text {trad }}: \mathfrak{g} \rightarrow L_{\mathbb{R}}$ the trace-representation $\left(\nabla_{\text {trad }}\right)_{a}=\operatorname{tr}\left(\operatorname{ad}_{a}\right) \cdot \mathrm{id}$. Then the top group of cohomology $H_{\text {trad }}^{n}(\mathfrak{g})$ of $\mathfrak{g}$ with respect to $\nabla_{\text {trad }}$ is nonzero, $H_{\text {trad }}^{n}(\mathfrak{g}) \cong \mathbb{R}$, and if the multiplication of reals is compatible with respect to $\left(\nabla_{1}, \nabla_{2}, \nabla_{\text {trad }}\right)$ then the exterior multiplication $\wedge: \Lambda^{r} \mathfrak{g}^{*} \times \Lambda^{n-r} \mathfrak{g}^{*} \rightarrow$ $\Lambda^{n} \mathfrak{g}^{*} \cong \mathbb{R}$ yields the induced nondegenerate pairing in cohomology

$$
H_{\nabla_{1}}^{i}(\mathfrak{g}) \times H_{\nabla_{2}}^{n-i}(\mathfrak{g}) \rightarrow H_{\text {trad }}^{n}(\mathfrak{g}) \cong \mathbb{R}
$$

i.e.

$$
H_{\nabla_{1}}^{i}(\mathfrak{g}) \stackrel{\cong}{\longrightarrow}\left(H_{\nabla_{2}}^{n-i}(\mathfrak{g})\right)^{*}
$$

In particular, for $\left(\nabla_{1}, \nabla_{2}, \nabla_{\text {trad }}\right)=\left(0, \nabla_{\text {trad }}, \nabla_{\text {trad }}\right)$ we obtain

$$
H^{i}(\mathfrak{g}) \stackrel{\cong}{\rightrightarrows}\left(H_{\text {trad }}^{n-i}(\mathfrak{g})\right)^{*} .
$$

For unimodular Lie algebra $\mathfrak{g}$ the usual Poincaré duality is obtained in this way.

- Let $A=\left(A, \llbracket \cdot, \cdot \rrbracket, \#_{A}\right)$ be a Lie algebroid on $M$ and

$$
Q_{A}=\Lambda^{\text {top }} A \otimes \Lambda^{\text {top }} T^{*} M
$$

the line vector bundle with canonical Evens-Lu-Weinstein representation [E-L-W],

$$
D_{\gamma}(Y \otimes \varphi)=L_{\gamma}(Y) \otimes \varphi+Y \otimes L_{\#_{A}(\gamma)}(\varphi) .
$$

To consider non-oriented manifolds we modify it into

$$
Q_{A}^{o r}=Q_{A} \otimes \text { or }(M)
$$

and

$$
D^{o r}=D \otimes \partial_{A}^{o r}
$$


tensoring by the orientation bundle and its flat structure $\left(\left(\partial_{A}^{o r}\right)_{\gamma} \sigma=\left(\partial^{o r}\right)_{\#_{A}(\gamma)} \sigma\right.$, $\sigma \in \Gamma(\operatorname{or}(M)), \#_{A}: A \rightarrow T M$ is the anchor of $\left.A\right)$. For transitive Lie algebroids with $n$-dimensional isotropy Lie algebras and multiplications by reals $(M \times \mathbb{R}) \otimes$ $Q_{A}^{o r} \rightarrow Q_{A}^{o r}$ the induced pairing in cohomology

$$
H^{j}(A) \times H_{D^{o r}, c}^{m+n-j}\left(A, Q_{A}^{o r}\right) \rightarrow H_{D^{o r}, c}^{m+n}\left(A, Q_{A}^{o r}\right) \rightarrow \mathbb{R}
$$

is nondegenerate, i.e. $H_{D^{o r}, c}^{m+n}\left(A, Q_{A}^{\text {or }}\right) \cong \mathbb{R}$ and

$$
H^{j}(A) \cong\left(H_{D^{o r}, c}^{m+n-j}\left(A, Q_{A}^{o r}\right)\right)^{*} \text {. }
$$

SECOND CYCLE shows the uniqueness of the representation for which the top group of compactly supported cohomology is not zero (Theorems 2.10, 3.5, $7.10)$

- $H_{\nabla, c}^{m}(M, \xi) \neq 0$ if and only if $(\xi, \nabla)$ is, up to isomorphy, the orientation flat line bundle $\left(\right.$ or $\left.(M), \partial^{\text {or }}\right)$. In particular, for oriented manifold, $H_{\nabla, c}^{m}(M, \xi) \neq 0$ if and only if $(\xi, \nabla)$ is, up to isomorphy, the trivial flat line bundle $(M \times \mathbb{R}, \partial)$.

- For an $n$-dimensional Lie algebra $\mathfrak{g}$ the trace-representation $\nabla_{\text {trad }}$ is the unique line representation $\nabla$ for which $H_{\nabla}^{n}(\mathfrak{g}) \neq 0$.

- Let $A$ be a transitive Lie algebroid and $\nabla$ a representation of $A$ in a line vector bundle $\xi$. Then $H_{\nabla, c}^{m+n}(A, \xi) \neq 0$ if and only if $(\xi, \nabla)$ is, up to isomorphy, the E-L-W-representation $\left(Q_{A}^{o r}, D^{o r}\right)$.

In conclusion we obtain a full classification of transitive Lie algebroids for which the algebra of real cohomologies with trivial coefficients satisfies the Poincaré duality.

- If $A$ is a transitive Lie algebroid then the following conditions are equivalent:

- $H_{c}^{m+n}(A) \neq 0$,

- $H_{c}^{m+n}(A) \cong \mathbb{R}$ and $H^{j}(A) \cong\left(H_{c}^{m+n-j}(A)\right)^{*}$,

- $A$ is orientable vector bundle and the modular class of $A$ is zero, $\theta_{A}=0$.

In particular,

- For an orientable manifold $M$ we have: $H_{c}^{m+n}(A) \neq 0$ if and only if $A$ is a TUIO-Lie algebroid [K2], i.e. the adjoint Lie Algebra Bundle $\boldsymbol{g}=\operatorname{ker} \#_{A}$ is oriented and there is a global nonsingular section $\varepsilon \in \Gamma\left(\Lambda^{n} \boldsymbol{g}\right)$ invariant with respect to the adjoint representaion.

The above theorem for a compact oriented manifold $M$ and 1-rank adjoint LAB $\boldsymbol{g}=M \times \mathbb{R}$ was proved earlier in $[\mathrm{K}-\mathrm{K}-\mathrm{K}-\mathrm{W}]$.

To prove Theorem 7.10 we use Theorem 4.4 concerning a pairing $\cdot:{ }^{1} A \times{ }^{2} A \rightarrow{ }^{3} A$ between graded filtered differential $\mathbb{R}$-vector spaces and theirs spectral sequnces. Roughly speaking, if the second terms ${ }^{r} E_{2}^{j, i}$ live in the rectangular $0 \leq j \leq m$, $0 \leq i \leq n,{ }^{3} E_{2}^{(m+n)}={ }^{3} E_{2}^{m, n} \cong \mathbb{R}$ and the multiplication of the second terms $\langle\cdot, \cdot\rangle_{2}:{ }^{1} E_{2}^{(j)} \times{ }^{2} E_{2}^{(m+n-j)} \rightarrow{ }^{3} E_{2}^{m, n} \cong \mathbb{R}$ is nondegenerate in the sense that ${ }^{1} E_{2}^{(j)} \cong\left({ }^{2} E_{2}^{(m+n-j)}\right)^{*}$, then the cohomology pairing for cohomologies of spaces is nondegenerate as well, i.e. ${ }^{3} H^{m+n} \cong \mathbb{R}$ and ${ }^{1} H^{j} \cong\left({ }^{2} H^{m+n-j}\right)^{*}$. We must stress that the spaces ${ }^{r} E_{2}^{j, i}$ may be infinite dimensional. 


\section{Non-DEgenerate PAIRINGS For TWISted COHOMOLOGY OF A MANifold}

Many of the facts from this section belong to "the folklore". We call 1-dimensional vector bundles line bundles.

2.1. Twisted cohomology, elementary properties

Let $M$ be an $m$-dimensional paracompact manifold and $\xi$ a vector bundle of rank $p$ and $\nabla_{X} \nu, X \in \mathfrak{X}(M), \nu \in \Gamma(\xi)$, a flat covariant derivative on $M$ in $\xi$.

$(\bullet)$ The differerential equation $\nabla \nu=0$ (with respect to the local section $\nu$ of $\xi)$ is locally uniquelly integrable.

The local section $\nu$ satisfying $\nabla \nu=0$ is called $\nabla$-constant (or sometimes $\nabla$ invariant). To set a flat covariant derivative $\nabla$ is equivalent to set local trivializations $\left\{\left(U_{\alpha}, \varphi_{\alpha}\right)\right\}$ relative to which the transitive functions are locally constant which is, in turn, equivalent to set a homomorphism of Lie algebroids $\nabla: T M \rightarrow A(\xi)$ where $A(\xi)$ is the Lie algebroid of $\xi$. The flat bundles $(\xi, \nabla)$ form a category with morphisms $F:\left(\xi_{1}, \nabla_{1}\right) \rightarrow\left(\xi_{2}, \nabla_{2}\right)$ being linear isomorphisms $F: \xi_{1} \rightarrow \xi_{2}$ compatible with flat covariant derivatives $\left(\nabla_{1}, \nabla_{2}\right)$, i.e. for which $F\left(\nabla_{1, X} \nu\right)=\nabla_{2, X}(F \nu)$. We write also

$$
\left(\xi_{1}, \nabla_{1}\right) \stackrel{F}{\sim}\left(\xi_{2}, \nabla_{2}\right)
$$

or briefly $\nabla_{1} \stackrel{F}{\sim} \nabla_{2}$.

Two flat line bundles over a connected manifold are isomorphic if and only if they have the same holonomy homomorphism $h: \pi_{1}(M, x) \rightarrow G L(\mathbb{R}, 1)$. For a flat vector bundle $(\xi, \nabla)$ the differential operator $d_{\nabla}$ of the degree 1 for $\xi$-valued differential forms $\Omega^{*}(M, \xi)$ is defined by the standard formula

$$
\begin{aligned}
& d_{\nabla}(\phi)\left(X_{0}, \ldots, X_{q}\right) \\
& =\sum_{i}(-1)^{i} \nabla_{X_{i}}\left(\phi\left(X_{0}, \ldots \hat{\imath} \ldots, X_{q}\right)\right)+\sum_{i<j}(-1)^{i+j} \phi\left(\left[X_{i}, X_{j}\right], X_{0}, \ldots \hat{\imath} \ldots \hat{\jmath} \ldots, X_{q}\right) .
\end{aligned}
$$

Let $\sigma_{\alpha}^{1}, \ldots, \sigma_{\alpha}^{p}$ be local sections of $\xi$ over $U_{\alpha}$ corresponding to the standard basis $e^{1}, \ldots, e^{p} \in \mathbb{R}^{p}$ under the trivialization $\varphi_{\alpha}, \sigma_{\alpha}^{i}(x)=\varphi_{\alpha, x}\left(e^{i}\right)$. The local sections $\sigma_{\alpha}^{i}$ are $\nabla$-constant, $\nabla \sigma_{\alpha}^{i}=0$. Over $U_{\alpha}$ a $\xi$-valued $q$-form $\phi$ can be written as $\sum \phi^{i} \otimes \sigma_{\alpha}^{i}$, $\phi^{i} \in \Omega^{q}\left(U_{\alpha}\right)$ and we have $d_{\nabla}\left(\sum \phi^{i} \otimes \sigma_{\alpha}^{i}\right)=\sum d_{d R}\left(\phi^{i}\right) \otimes \sigma_{\alpha}^{i}$. The flatness of $\nabla$ implies that $d_{\nabla}$ is a differential operator, $d_{\nabla}^{2}=0$, therefore $\Omega^{*}(M, \xi)$ is a differential complex and the (twisted) cohomology

$$
H_{\nabla}^{*}(M, \xi)=H\left(\Omega^{*}(M, \xi), d_{\nabla}\right)
$$

makes sense. By the definition the 0-group of cohomology can be written as

$$
H_{\nabla}^{0}(M, \xi)=\left\{\nu \in \Gamma(\xi) ; \nabla_{X} \nu=0 \text { for all } X \in \mathfrak{X}(M)\right\} .
$$

$(\bullet \bullet)$ If $(\xi, \nabla)$ is a line nontrivial flat vector bundle then according to (•) above $H_{\nabla}^{0}(M, \xi)=0$.

If $\nabla$ is a flat covariant derivative in a vector bundle $\xi$ and $\omega \in \Omega^{1}(M)$ is a closed real 1-form, then

$$
\nabla_{X}^{\omega} \nu=\nabla_{X} \nu+\omega(X) \cdot \nu
$$

is a flat covariant derivative as well. If $\xi$ is a line bundle and $\nabla$ and $\nabla_{1}$ are two flat covariant derivatives then there exists a closed 1 -form $\omega$ such that $\nabla_{1}=\nabla^{\omega}$.

Each flat covariant derivative $\nabla$ in the trivial vector bundle $M \times R$ is of the form $\partial^{\omega}$ for some closed 1 -form $\omega$ ( $\partial$ is the standard covariant derivative in the 
trivial vector bundle $M \times R$ defined by differentiation of functions $\partial_{X}(f)=X(f)$ ). Differential operator $d_{\partial \omega}$ is given directly by

$$
d_{\partial^{\omega}}(\phi)=d_{d R} \phi+\omega \wedge \phi .
$$

The operator $d_{\partial \omega}$ is in the literature denoted rather by $d_{\omega}$ than by $d_{\partial \omega}[\mathrm{G}-\mathrm{L}],[\mathrm{H}-\mathrm{R}]$ and the cohomology space $H_{\partial \omega}(M, M \times \mathbb{R})$ is denoted by $H_{\omega}(M)$. If $\omega=0$ the usual de Rham cohomology of $M$ is obtained. It is easy to see that

$$
H_{\omega}^{0}(M)=\left\{\begin{array}{l}
0 \Longleftrightarrow \omega \text { is nonexact, } \\
\mathbb{R} \Longleftrightarrow \omega \text { is exact. }
\end{array}\right.
$$

The space of $\xi$-valued $q$-forms with compact support $\Omega_{c}^{*}(M, \xi)$ is a differential complex as well and we have the compactly supported cohomology $H_{\nabla, c}^{*}(M, \xi)$. If $\left(\xi_{1}, \nabla_{1}\right) \stackrel{F}{\sim}\left(\xi_{2}, \nabla_{2}\right)$ then $F_{*}: \Omega^{*}\left(M, \xi_{1}\right) \rightarrow \Omega^{*}\left(M, \xi_{2}\right)$ commutes with the differential operators $d_{\nabla_{1}}$ and $d_{\nabla_{2}}$ and gives rise to an isomorphism in cohomology $F_{\#}: H_{\nabla_{1}}^{*}\left(M, \xi_{1}\right) \rightarrow H_{\nabla_{2}}^{*}\left(M, \xi_{2}\right)$. Analogously, for compact supports, we have an isomorphism $F_{\#, c}: H_{\nabla_{1}, c}^{*}\left(M, \xi_{1}\right) \rightarrow H_{\nabla_{2}, c}^{*}\left(M, \xi_{2}\right)$.

For an open subset $U \subset M$ we have the restricted flat covariant derivative $\nabla_{U}$ on $U$ in the vector bundle $\xi_{U}$ and the twisted cohomology $H_{\nabla}(U, \xi)$ and $H_{\nabla, c}(U, \xi)$ are defined. Similarly as in the case of real coefficients (see for example [B-T]) we can obtain the short exact Mayer-Vietoris sequences $\left(U_{1}, U_{2} \subset M\right.$ are open subset, $U=U_{1} \cup U_{2}$, and $\left.U_{12}=U_{1} \cap U_{2}\right)$

$$
0 \rightarrow \Omega^{*}(U, \xi) \stackrel{\alpha}{\rightarrow} \Omega^{*}\left(U_{1}, \xi\right) \oplus \Omega^{*}\left(U_{2}, \xi\right) \stackrel{\beta}{\rightarrow} \Omega^{*}\left(U_{12}, \xi\right) \rightarrow 0
$$

and

$$
0 \leftarrow \Omega_{c}^{*}(U, \xi) \stackrel{\alpha_{c}}{\leftarrow} \Omega_{c}^{*}\left(U_{1}, \xi\right) \oplus \Omega_{c}^{*}\left(U_{2}, \xi\right) \stackrel{\beta_{c}}{\leftarrow} \Omega_{c}^{*}\left(U_{12}, \xi\right) \leftarrow 0 .
$$

They give rise to long exact sequences in cohomology

$$
\rightarrow H_{\nabla}^{q}(U, \xi) \stackrel{\alpha_{\#}}{\rightarrow} H_{\nabla}^{q}\left(U_{1}, \xi\right) \oplus H_{\nabla}^{q}\left(U_{2}, \xi\right) \stackrel{\beta_{\#}}{\rightarrow} H_{\nabla}^{q}\left(U_{12}, \xi\right) \stackrel{\partial^{q}}{\rightarrow} H_{\nabla}^{q+1}(U, \xi) \rightarrow
$$

and

$$
\leftarrow H_{\nabla, c}^{q}(U, \xi) \stackrel{\alpha_{c \#}}{\leftarrow} H_{\nabla, c}^{q}\left(U_{1}, \xi\right) \oplus H_{\nabla, c}^{q}\left(U_{2}, \xi\right) \stackrel{\beta_{c \#}}{\longleftarrow} H_{\nabla, c}^{q}\left(U_{12}, \xi\right) \stackrel{\partial_{c}^{q}}{\leftarrow} H_{\nabla, c}^{q+1}(U, \xi) \leftarrow
$$

Remark 2.1. There is a natural isomorphism $H_{\nabla}^{*}(M, \xi) \cong H_{I(\nabla)}^{*}(\mathfrak{U}, \xi)$ of $H_{\nabla}^{*}(M, \xi)$ with $H_{I(\nabla)}^{*}(\mathfrak{U}, \xi)$, the cohomology of $M$ in the sheaf $I(\nabla)$ of local $\nabla$-constant sections of $\xi$. In other words, $H_{\nabla}^{*}(M, \xi)$ are cohomology of $M$ with local system of coefficients.

2.2. Orientation flat bundle and its characterization. Let $\left\{\left(U_{\alpha}, x_{a}\right)\right\}$ be a coordinate open cover for the manifold $M$, with transition functions $g_{\alpha \beta}=x_{\alpha} \circ x_{\beta}^{-1}$. Take the orientation bundle or $(M)$, i.e. the line bundle on $M$ with a distinguished system of local trivializations $\left\{\varphi_{\alpha}\right\}$ such that the transition functions are equal to $\operatorname{sgn} J\left(g_{\alpha \beta}\right)$ [B-T]. Let $\left\{e_{\alpha}\right\}$ be a family of local sections corresponding to 1 under the trivializations $\left\{\varphi_{\alpha}\right\}, e_{\alpha}(x)=\varphi_{\alpha, x}(1)$. In the bundle or $(M)$ there exists exactly one flat covariant derivative $\partial^{o r}$ such that $e_{\alpha}$ are $\partial^{o r}$-constant, $\partial^{o r}\left(e_{\alpha}\right)=0$. The notation $e_{\alpha}$ and $\partial^{o r}$ is valid in the whole paper.

The flat orientation bundle ( $o r(M), \partial^{o r}$ ) is characterized by the holonomy homomorphism $s: \pi_{1}\left(M, x_{0}\right) \rightarrow \mathbb{Z}_{2} \subset G L(\mathbb{R}, 1)$ that can be identified with monodromy to the [o co tu chodzi?] group of the local orientations in the fixed point $x_{0}$ which also is $\mathbb{Z}_{2}$. 
In the sequel it will be useful to give other characterization of the flat orientation bundle.

Proposition 2.2. Let $(\xi, \nabla)$ be a flat line bundle. The following conditions are equivalent:

(a) $(\xi, \nabla) \cong\left(\right.$ or $\left.(M), \partial^{o r}\right)$,

(b) there exists a collection of local sections $\left\{\sigma_{\alpha}\right\}$ of $\xi$ such that $\sigma_{a}$ are $\nabla$ constant and the transition functions are equal to $\operatorname{sgn} J\left(g_{\alpha \beta}\right)$,

(c) $\left(\right.$ or $\left.(M) \otimes \xi, \partial^{o r} \otimes \nabla\right) \cong(M \times \mathbb{R}, \partial)$,

(d) there exists a global nonsingular section $t \in \Gamma(\operatorname{or}(M) \otimes \xi)$ which is $\partial^{\text {or }} \otimes \nabla$ constant.

Proof. Equivalences (a) $\Leftrightarrow(\mathrm{b})$ and $(\mathrm{c}) \Leftrightarrow(\mathrm{d})$ are evident by definition.

(b) $\Rightarrow$ (c) The linear homomorphism $F:$ or $(M) \otimes \xi \rightarrow M \times \mathbb{R}$ defined by $F\left(e_{\alpha} \otimes \sigma_{\alpha}\right)=$ 1 is a well defined linear isomorphism compatible with $\left(\partial^{\text {or }} \otimes \nabla, \partial\right)$.

(d) $\Rightarrow$ (b) Locally $t=e_{\alpha} \otimes \sigma_{\alpha}$ for some local nonsingular sections $\sigma_{\alpha}$ of $\xi$. Since

$$
0=\partial^{o r} \otimes \nabla\left(e_{\alpha} \otimes \sigma_{\alpha}\right)=\partial^{o r} \otimes \sigma_{\alpha}+e_{\alpha} \otimes \nabla \sigma_{\alpha}=e_{\alpha} \otimes \nabla \sigma_{\alpha},
$$

it follows that $\sigma_{\alpha}$ are $\nabla$-constant and have the same transition functions $\operatorname{sgn} J\left(g_{\alpha \beta}\right)$.

The or $(M)$-valued $m$-differential forms are called densities. There exists an operator

$$
\int_{M}^{o r}: \Omega_{c}^{m}(M, \text { or }(M)) \rightarrow \mathbb{R}
$$

of the integration of densities and the Stoke's Theorem for densities holds

$$
\int_{M}^{o r} d_{\partial o r}(\omega)=0
$$

for $\omega \in \Omega_{c}^{m-1}(M$, or $(M))$ [B-T]. Hence it produces a linear operator

$$
\int_{M}^{o r, \#}: H_{\partial^{o r}, c}^{m}(M, \text { or }(M)) \rightarrow \mathbb{R}
$$

2.3. Pairings and cohomology, nondegeneracy. Now let $\left(\xi_{1}, \nabla_{1}\right),\left(\xi_{2}, \nabla_{2}\right)$, and $\left(\xi_{3}, \nabla_{3}\right)$ be three flat vector bundles. We say that $\left(\xi_{1}, \nabla_{1}\right)$ and $\left(\xi_{2}, \nabla_{2}\right)$ are paired to $\left(\xi_{3}, \nabla_{3}\right)$ if there is a bilinear homomorphism $F: \xi_{1} \times \xi_{2} \rightarrow \xi_{3}$ compatible with flat covariant derivatives $\left(\nabla_{1}, \nabla_{2}, \nabla_{3}\right)$, i.e. such that, for every $X \in \mathfrak{X}(M)$,

$$
\nabla_{3, X} F\left(\nu_{1}, \nu_{2}\right)=F\left(\nabla_{1, X} \nu_{1}, \nu_{2}\right)+F\left(\nu_{1}, \nabla_{2, X} \nu_{2}\right) .
$$

Then we write $F:\left(\xi_{1}, \nabla_{1}\right) \times\left(\xi_{2}, \nabla_{2}\right) \rightarrow\left(\xi_{3}, \nabla_{3}\right)$. From such a pairing one obtains a pairing $(\phi, \psi) \mapsto \phi \wedge \psi:=F_{*}(\phi, \psi)$ of $\Omega^{q}\left(M, \xi_{1}\right)$ and $\Omega^{r}\left(M, \xi_{2}\right)$ to $\Omega^{q+r}\left(M, \xi_{3}\right)$ fullfilling the equality

$$
d_{\nabla_{3}} F_{*}(\phi, \psi)=F_{*}\left(d_{\nabla_{1}} \phi, \psi\right)+(-1)^{\operatorname{deg} \phi} F_{*}\left(\phi, d_{\nabla_{2}} \psi\right) .
$$

Clearly, $\phi \wedge \psi:=F_{*}(\phi, \psi)$ is the usual wedge product of differential forms with $F$-multiplication of values, see [G-H-V, Vol.II]. The pairing of differential forms induces a pairing of cohomology classes

$$
F_{\#}: H_{\nabla_{1}}^{*}\left(M, \xi_{1}\right) \times H_{\nabla_{2}}^{*}\left(M, \xi_{2}\right) \rightarrow H_{\nabla_{3}}^{*}\left(M, \xi_{3}\right)
$$

as well as the pairing for compact supports

$$
F_{\#, c}: H_{\nabla_{1}}^{*}\left(M, \xi_{1}\right) \times H_{\nabla_{2}, c}^{*}\left(M, \xi_{2}\right) \rightarrow H_{\nabla_{3}, c}^{*}\left(M, \xi_{3}\right) .
$$


Consider two flat vector bundles $\left(\xi_{1}, \nabla_{1}\right),\left(\xi_{2}, \nabla_{2}\right)$ and a pairing

$$
F:\left(\xi_{1}, \nabla_{1}\right) \times\left(\xi_{2}, \nabla_{2}\right) \rightarrow\left(\text { or }(M), \partial^{\text {or }}\right) .
$$

For an open subset $U \subset M$ we define a pairing

$$
\int_{U}^{o r, \#} \circ F_{\#}: H_{\nabla_{1}}^{q}\left(U, \xi_{1}\right) \times H_{\nabla_{2}, c}^{m-q}\left(U, \xi_{2}\right) \stackrel{F_{\#}}{\rightarrow} H_{\partial^{o r}, c}^{m}(U, \text { or }(M)) \stackrel{\int_{U}^{o r, \#}}{\rightarrow} \mathbb{R},
$$

and the Poincaré linear homomorphism

$$
D_{U}^{q}: H_{\nabla_{1}}^{q}\left(U, \xi_{1}\right) \rightarrow\left(H_{\nabla_{2}, c}^{m-q}\left(U, \xi_{2}\right)\right)^{*}, \quad D_{U}^{q}([\Phi])([\Psi])=\int_{U}(\Phi \wedge \Psi) .
$$

Similarly as in the case of real coefficients we check that the family of Poincaré homomorphisms $\left\{D_{U}^{q}\right\}$ induces a map from the long exact sequences in cohomology to the long exact sequences in compactly supported cohomology (the symbols of vector bundles $\xi_{1}$ and $\xi_{2}$ in the diagram below are ommitted and the sign \pm is equal precisely to $\left.(-1)^{q+1}\right)$

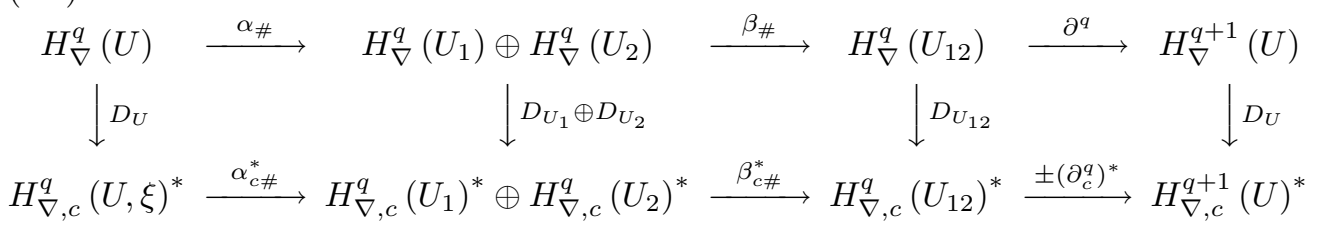

For an infinite disjoint open subsets $U=\coprod U_{i}$ we deduce that $D_{U}$ can be identifying with $\prod D_{U_{i}}$.

Theorem 2.3. Assume that $M$ is connected. If pairing (2.6) is nondegenerate at least one point then the cohomology pairing

$$
\int_{M}^{o r, \#} \circ F_{\#}: H_{\nabla_{1}}^{q}\left(M, \xi_{1}\right) \times H_{\nabla_{2}, c}^{m-q}\left(M, \xi_{2}\right) \stackrel{F_{\#}}{\rightarrow} H_{\partial^{\text {or }, c}}^{m}(M, \text { or }(M)) \stackrel{\int_{M}^{o r, \#}}{\rightarrow} \mathbb{R},
$$

is also nondegenerate in the sense that

$$
D_{M}^{q}: H_{\nabla_{1}}^{q}\left(M, \xi_{1}\right) \cong\left(H_{\nabla_{2}, c}^{m-q}\left(M, \xi_{2}\right)\right)^{*}
$$

is an isomorphism, $q \in\{0,1, \ldots, m\}$.

Proof. We can use the standard method from [G-H-V, Vol.I] (or a slightly modified method by using Riemannian structure and properties of geodesically convex neighbourhoods, [B-T], [S]. According to [G-H-V, Vol.I, Prop.II, p.16] and the commutativity of diagram (2.7) and remark on infinite disjoint open subsets we need only to prove the theorem for the manifold $M=\mathbb{R}^{m}$.

Each vector bundle $\xi$ over $\mathbb{R}^{m}$ is trivial, each flat covariant derivative $\nabla$ has trivial holonomy, so the differential equation $\nabla \nu=0$ is globally integrable. Therefore for an arbitrary point $x_{0} \in M$ there exists an isomorphism of flat vector bundles

$$
\varphi:(\xi, \nabla) \rightarrow\left(\mathbb{R}^{m} \times \xi_{x_{0}}, \partial\right)
$$

where by $\partial$ is denoted the standard flat covariant derivative $\partial_{X} f=X(f)$.

(Remark: for the line bundle $\xi$ the isomorphism $\varphi$ can be given directly as follows. For $\xi=\mathbb{R}^{m} \times \mathbb{R}$ any flat covariant derivative $\nabla$ is of the form $\nabla_{X} f=\partial_{X} f+\partial_{X}(\alpha) \cdot f$ for some function $\alpha$. Then $\varphi(f)=e^{-\alpha} f$ is a required isomorphism.) 
The isomorphism $\varphi$ gives rise to an isomorphism in cohomology

$$
\varphi_{\#}: H_{\nabla}\left(\mathbb{R}^{m}, \xi\right) \stackrel{\cong}{\rightarrow} H_{d R}\left(\mathbb{R}^{m}, \xi_{x_{0}}\right),
$$

especially for the zero level

$$
H_{\nabla}^{0}\left(\mathbb{R}^{m}, \xi\right) \underset{\cong}{\stackrel{\varphi_{\#}^{0}}{\cong}} H_{d R}^{0}\left(\mathbb{R}^{m}, \xi_{x_{0}}\right) \stackrel{\rho}{\cong} \xi_{x_{0}} .
$$

On the other hand, the isomorphism $\varphi$ also gives rise to an isomorphism in compactly supported cohomology $\varphi_{\#, c}: H_{\nabla, c}\left(\mathbb{R}^{m}, \xi\right) \stackrel{\cong}{\rightrightarrows} H_{d R, c}\left(\mathbb{R}^{m}, \xi_{x_{0}}\right)$, especially for the top level

$$
\varphi_{\#, c}^{m}: H_{\nabla, c}^{m}\left(\mathbb{R}^{m}, \xi\right) \stackrel{\varphi_{\#, c}^{m}}{\cong} H_{d R, c}^{m}\left(\mathbb{R}^{m}, \xi_{x_{0}}\right) \underset{\cong}{\stackrel{\rho_{c}}{\cong}} \xi_{x_{0}},
$$

where $\rho_{c}$ is defined by the formula

$$
\rho_{c}\left(\left[\sum_{i} f^{i} \cdot \Delta \otimes v_{i}\right]\right)=\sum_{i}\left(\int_{\mathbb{R}^{m}} f^{i}\right) \cdot e^{i}
$$

where $v_{i}$ is a basis of $\xi_{x_{0}}, \Delta$ is a determinant function on $\mathbb{R}^{m}$ and $f^{i} \in C_{c}^{\infty}\left(\mathbb{R}^{m}\right)$ are functions with compact support. $\rho_{c}$ is independent of the choice of the basis $v_{i}$ and fulfils the equality $\rho_{c}([f \cdot \Delta \otimes v])=\left(\int_{\mathbb{R}^{m}} f\right) \cdot v, f \in C_{c}^{\infty}\left(\mathbb{R}^{m}\right), v \in \xi_{x_{0}}$.

Now take flat vector bundles $\left(\xi_{i}, \nabla_{i}\right)$ on $\mathbb{R}^{m}$ and linear isomorphisms $\varphi_{i}:\left(\xi_{i}, \nabla_{i}\right) \rightarrow$ $\left(\mathbb{R}^{m} \times\left(\xi_{i}\right)_{x_{0}}, \partial\right)$. For any pairing $F:\left(\xi_{1}, \nabla_{1}\right) \times\left(\xi_{2}, \nabla_{2}\right) \rightarrow\left(\xi_{3}, \nabla_{3}\right)$ we get easily the commutative diagram

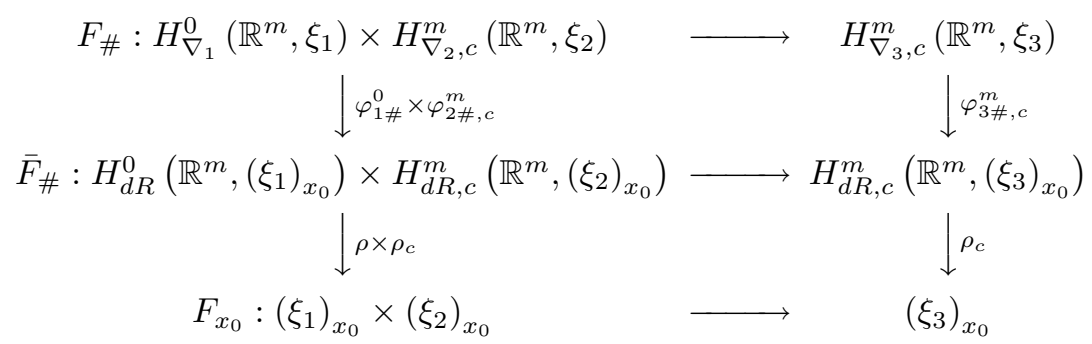

where the middle pairing comes from the "constant" pairing

$$
\bar{F}:\left(\mathbb{R}^{m},\left(\xi_{1}\right)_{x_{0}}\right) \times\left(\mathbb{R}^{m},\left(\xi_{2}\right)_{x_{0}}\right) \rightarrow\left(\mathbb{R}^{m},\left(\xi_{3}\right)_{x_{0}}\right), \quad F_{x}=F_{x_{0}} .
$$

To prove the theorem take $\left(\xi_{3}, \nabla_{3}\right)=\left(\right.$ or $\left.(M), \partial^{o r}\right)$ and choose a point $x_{0}$ such that $F_{x_{0}}$ is nondegenerate.

2.4. Applications of the nondegenerate cohomology pairing. Now we give a number of applications of Theorem 2.3.

Example 2.4. For a connected orientable manifold $M$ and the trivial flat vector bundles $\left(\xi_{i}, \nabla_{i}\right)=(M \times \mathbb{R}, \partial)$ and the multiplication of reals $\cdot: \mathbb{R} \times \mathbb{R} \rightarrow \mathbb{R}$ we obtain the classical Poincaré duality $H^{j}(M) \times H_{c}^{m-j}(M) \rightarrow H_{c}^{m}(M) \rightarrow \mathbb{R}$. Especially $H_{c}^{m}(M)=\mathbb{R}$ and $H^{j}(M) \cong\left(H_{c}^{m-j}(M)\right)^{*}$.

Example 2.5. More generally, for arbitrary connected manifold $M$ taking $\left(\xi_{1}, \nabla_{1}\right)=$ $(M \times \mathbb{R}, \partial)$ and $\left(\xi_{2}, \nabla_{2}\right)=\left(\right.$ or $\left.(M), \partial^{\text {or }}\right)$ and the multiplication by reals $F:(M \times \mathbb{R}) \times$ or $(M) \rightarrow$ or $(M)$ we get the Poincaré duality also for nonorientable manifold [B-T]. Especially operator $(2.4)$ is an isomorphism, $H_{\partial^{o r}, c}^{m}(M$, or $(M)) \cong \mathbb{R}$.

Example 2.6. [G-L], [H-R] Let $M$ be an oriented connected manifold. The following conditions are equivalent: 
(1) $H_{\omega, c}^{m}(M)=0$,

(2) $H^{1}(M) \ni[\omega] \neq 0$.

If $[\omega]=0$ then $H_{\omega}^{m}(M)=\mathbb{R}$. Indeed, consider multiplication by reals $F$ : $(M \times \mathbb{R}) \times(M \times \mathbb{R}) \rightarrow M \times \mathbb{R}$. This pairing is nondegererate and compatible with $\left(\partial^{-\omega}, \partial^{\omega}, \partial\right)$. By Theorem 2.3 we get the nondegenerate pairing $H_{-\omega}^{p}(M) \times$ $H_{\omega, c}^{m-p}(M) \rightarrow H_{c}^{m}(M) \cong \mathbb{R}$. In particular, we get $H_{-\omega}^{0}(M)=\left(H_{\omega, c}^{m}(M)\right)^{*}$, so all follows from (2.3).

Each flat covariant derivative in or $(M)$ is of the form $\left(\partial^{\text {or }}\right)^{\omega}$ for a closed 1-form $\omega$. Concider the multiplications by reals $F:(M \times \mathbb{R}) \times$ or $(M) \rightarrow$ or $(M)$. Then we easily get:

Example 2.7. For any connected manifold $M$ (oriented or not) the following conditions are equivalent:

(1) $H_{\left(\partial^{o r}\right)^{\omega}, c}^{m}(M, o r(M))=0$,

(2) $H^{1}(M) \ni[\omega] \neq 0$.

$$
\text { If }[\omega]=0 \text { then } H_{\left(\partial^{o r}\right)^{\omega}, c}^{m}(M, \text { or }(M))=\mathbb{R} \text {. }
$$

The next applications are given in the following propositions.

Proposition 2.8. If $M$ is orientable and $\xi$ is an arbitrary line nonorientable (i.e. nontrivial) vector bundle then for any flat covariant derivative $\nabla$ in $\xi$

$$
H_{\nabla, c}^{m}(M, \xi)=0 .
$$

Proof. Indeed, consider the natural nondegenerate pairing

$$
F:(\xi, \nabla) \times(\xi, \nabla) \rightarrow(\xi \otimes \xi, \nabla \otimes \nabla), \quad(\nu, \mu) \mapsto \nu \otimes \mu,
$$

and any linear isomorphism $\varphi: \xi \otimes \xi \rightarrow M \times \mathbb{R}$. The latter transforms the flat covariant derivative $\nabla \otimes \nabla$ to the $\partial^{\omega}$ for some closed 1-form $\omega$. We recall that $(\nabla \otimes \nabla)_{X}(\nu \otimes \mu)=\nabla_{X} \nu \otimes \mu+\nu \otimes \nabla_{X} \mu$. Then the pairing $\varphi \circ F: \xi \times \xi \rightarrow M \times \mathbb{R}$ is compatible with $\left(\nabla, \nabla, \partial^{\omega}\right)$ and, in consequence, with $\left(\nabla^{-\omega}, \nabla, \partial\right)$ (for $\nabla^{-\omega}$ see (2.2)). By Theorem 2.3 we have the nondegenerate pairing

$$
H_{\nabla^{-\omega}}^{0}(M, \xi) \times H_{\nabla, c}^{m}(M, \xi) \rightarrow H_{c}^{m}(M) \stackrel{\int_{M}}{\rightarrow} \mathbb{R} .
$$

In consequence we obtain by the nontriviality of $\xi$ and observation $(\bullet)$ from section 2.1

$$
0=H_{\nabla^{-\omega}}^{0}(M, \xi)=\left(H_{\nabla, c}^{m}(M, \xi)\right)^{*}
$$

which imply $H_{\nabla, c}^{m}(M, \xi)=0$.

Proposition 2.9. If $\xi$ is a line bundle not isomorphic to or $(M)$ then for arbitrary flat covariant derivative $\nabla$ in $\xi$ we have

$$
H_{\nabla, c}^{m}(M, \xi)=0 .
$$

Proof. Indeed, fix a linear isomorphism

$$
\varphi: \xi \otimes \xi \rightarrow M \times \mathbb{R}
$$

Such isomorphism $\varphi$ exists since $\xi \otimes \xi$ is orientable line vector bundle, therefore, trivial. Let $\nabla \otimes \nabla \stackrel{F}{\sim} \partial^{\omega}$ for a closed 1-form $\omega$. Take the multiplication by reals

$$
\tau: \text { or }(M) \otimes(M \times \mathbb{R}) \rightarrow \text { or }(M)
$$


and notice that $\tau$ is compatible with $\left(\partial^{o r} \otimes \partial^{\omega},\left(\partial^{o r}\right)^{\omega}\right)$. Consider the canonical nondegenerate pairing $F:(\operatorname{or}(M) \otimes \xi) \times \xi \rightarrow$ or $(M) \otimes \xi \otimes \xi$ which is compatible with $\left(\partial^{o r} \otimes \nabla, \nabla, \partial^{o r} \otimes \nabla \otimes \nabla\right)$. The composition

$$
F^{\prime}:(\text { or }(M) \otimes \xi) \times \xi \stackrel{F}{\rightarrow} \text { or }(M) \otimes \xi \otimes \xi \stackrel{i d \otimes \varphi}{\longrightarrow} \text { or }(M) \otimes(M \times \mathbb{R}) \stackrel{\tau}{\rightarrow} \text { or }(M),
$$

clearly, is nondegenerate and is compatible with $\left(\partial^{\text {or }} \otimes \nabla, \nabla,\left(\partial^{\text {or }}\right)^{\omega}\right)$. Therefore $F^{\prime}$ is compatible with $\left(\partial^{o r} \otimes \nabla^{-\omega}, \nabla, \partial^{o r}\right)$. According to Theorem 2.3 applied to $F^{\prime}$ we get

$$
H_{\partial^{o r} \otimes \nabla^{-\omega}}^{0}(M, \text { or }(M) \otimes \xi) \cong\left(H_{\nabla, c}^{m}(M, \xi)\right)^{*} .
$$

Since $\xi$ is not isomorphic to or $(M)$ the vector bundle or $(M) \otimes \xi$ is not trivial (indeed, if or $(M) \otimes \xi \cong M \times \mathbb{R}$ then or $(M) \cong \xi^{*} \cong \xi$ ) which produces $H_{\partial^{o r} \otimes \nabla^{-\omega}}^{0}(M$, or $(M) \otimes \xi)=0$ and further $H_{\nabla, c}^{m}(M, \xi)=0$.

Finally we have the main application.

Theorem 2.10. The following conditions are equivalent:

a) $H_{\nabla, c}^{m}(M, \xi) \neq 0$,

b) $H_{\nabla, c}^{m}(M, \xi)=\mathbb{R}$

c) $(\xi, \nabla) \sim\left(\right.$ or $\left.(M), \partial^{\text {or }}\right)$,

Proof. For c) $\Longrightarrow$ b) see Example 2.5 or $[\mathrm{B}-\mathrm{T}]$; b) $\Longrightarrow$ a) is evident. It remains to show that

a) $\Longrightarrow$ c). Keep the notation $\varphi$ and $\omega$ from the proof of the previous proposition. By the same reasoning we check

$$
H_{\partial^{o r} \otimes \nabla^{-\omega}}^{0}(M, \text { or }(M) \otimes \xi) \neq 0 .
$$

It means that or $(M) \otimes \xi$ is trivial and there exists a nonsingular global cros-section $\nu \in \Gamma(\operatorname{or}(M) \otimes \xi)$ which is $\partial^{o r} \otimes \nabla^{-\omega}$-constant. Express locally $\nu$ in the form $\nu=e_{\alpha} \otimes f_{\alpha}$ for some local sections $f_{\alpha}$ of $\xi$, for $e_{\alpha}$ see subsection 2.2. It is evident that $\left\{f_{\alpha}\right\}$ has the transition function equal to sgn $J g_{\alpha \beta}$ and that $\nabla^{-\omega} f_{\alpha}=0$, i.e. $\nabla_{X} f_{\alpha}=\omega(X) \cdot f_{\alpha}$. The formula $f=\varphi\left(f_{\alpha} \otimes f_{\alpha}\right)$ determines correctly a nonsingular function $f$. Since $\nabla \otimes \nabla \stackrel{\varphi}{\sim} \partial^{\omega}$ then $\partial_{X} f+\omega(X) \cdot f=\partial_{X}^{\omega} f=2 \cdot \omega(X) \cdot f$, one has

$$
\partial_{X} f=\omega(X) \cdot f .
$$

The global cros-section $\nu^{\prime}=\frac{1}{f} \nu$ is $\partial^{o r} \otimes \nabla$-constant. The proposition follows now from Proposition 2.2.

\section{A generalization of the Chern-Hirzebruch-Serre Lemma and APPLICATIONS TO COHOMOLOGY OF LIE ALGEBRAS}

We generalize Lemma 3 from [Ch-H-S] concerning Poincaré differentiation from algebras to pairings. The assumption on finite dimensionality is superfluous.

Lemma 3.1. Let $A_{s}=\bigoplus_{i=0}^{n} A_{s}^{i}, d_{s}: A_{s} \rightarrow A_{s}, s=1,2,3$, be three graded differential $\mathbb{R}$-vector spaces such that

(1) $d_{s}\left[A_{s}^{i}\right] \subset A_{s}^{i+1}$,

(2) $d_{s}^{2}=0$,

(3) $d_{3}\left[A_{3}^{n-1}\right]=0$.

(4) $A_{3}^{n} \cong \mathbb{R}, A_{3}^{i}=0$ for $i>n$. 
Let

be a pairing such that

$$
\cdot: A_{1} \times A_{2} \rightarrow A_{3}
$$

(5) $A_{1}^{i} \cdot A_{2}^{j} \subset A_{3}^{i+j}$,

(6) $d_{3}(x \cdot y)=d_{1} x \cdot y+(-1)^{\operatorname{deg} x} x \cdot d_{2} y$,

(7) $\cdot: A_{1}^{r} \times A_{2}^{n-r} \rightarrow A_{3}^{n} \cong \mathbb{R}, r=0,1, \ldots, n$ are nondegenerate $i n$ the sense that the induced mappings

$$
i_{r}: A_{1}^{r} \stackrel{\cong}{\longrightarrow}\left(A_{2}^{n-r}\right)^{*}
$$

are linear isomorphisms.

Then the induced homomorphisms in cohomology

$$
\cdot: H^{r}\left(A_{1}, d_{1}\right) \times H^{n-r}\left(A_{2}, d_{2}\right) \rightarrow H^{n}\left(A_{3}, d_{3}\right) \cong \mathbb{R}
$$

are nondegenerate as well, i.e. the induced linear homomorphism

$$
i_{r}^{\prime}: H^{r}\left(A_{1}, d_{1}\right) \rightarrow\left(H^{n-r}\left(A_{2}, d_{2}\right)\right)^{*}
$$

are linear isomorphisms.

Proof. The proof is identical with the original proof by Chern-Hirzebruch-Serre for an algebra and it is sufficient to check that

$$
i_{r}^{\prime}: H^{r}\left(A_{1}, d_{1}\right) \stackrel{\left(i_{r}\right)_{\#}}{\longrightarrow} H^{n-r}\left(A_{2}^{*}, d_{2}^{*}\right) \stackrel{\cong}{\longrightarrow} H^{n-r}\left(A_{2}, d_{2}\right),
$$

where $\left(A_{2}^{*}, d_{2}^{*}\right)$ denotes the dual complex.

Now we give some applications to the cohomology of Lie algebras with coefficients. Let $\mathfrak{g}$ be a real Lie algebra of dimension $n$ and let

$$
\nabla: \mathfrak{g} \rightarrow L_{\mathbb{R}}=\text { End } \mathbb{R} \cong \mathbb{R}
$$

be an arbitrary representation in 1 dimensional vector space. We will distinguish two representations

- $\nabla_{0}=0$

- $\left(\nabla_{\text {trad }}\right)_{a}=\operatorname{tr}\left(\operatorname{ad}_{a}\right) \cdot$ id .

We see that $\nabla_{0}=\nabla_{\text {trad }}$ if and only if $\mathfrak{g}$ is unimodular. Denote the differential with respect to $\nabla_{\text {trad }}$ by $d_{\text {trad }}$ and the cohomology of $\mathfrak{g}$ by $H_{\text {trad }}(\mathfrak{g})$. Straightforward computations show that $d_{\mathrm{trad}}^{n-1}=0$. Therefore

Proposition 3.2. $H_{\text {trad }}^{n}(\mathfrak{g})=\Lambda^{n} \mathfrak{g}^{*} \cong \mathbb{R}$ for every Lie algebra.

Let us notice the following

Remark 3.3. (1) Each representation $\nabla: \mathfrak{g} \rightarrow L_{\mathbb{R}}$ is equal to 0 on $\mathfrak{g}^{2}$ and, conversely, each linear homomorphism $\nabla: \mathfrak{g} \rightarrow L_{\mathbb{R}}$ such that $\nabla \mid \mathfrak{g}^{2}=0$ is a representation.

(2) The zero group of cohomology $H_{\nabla}^{0}(\mathfrak{g})=0$ if and only if $\nabla \neq 0$.

(3) The multiplication of reals $\cdot: \mathbb{R} \times \mathbb{R} \rightarrow \mathbb{R}$ is compatible with $\left(\nabla_{1}, \nabla_{2}, \nabla_{3}\right)$ if and only if $\nabla_{3}=\nabla_{1}+\nabla_{2}$.

Point (1) from the remark above implies that any linear combination of representations is a representation. Take an arbitrary representation $\nabla$ and put

$$
\nabla^{\prime}=\nabla_{\text {trad }}-\nabla
$$


Then the multiplication of reals is compatible with $\left(\nabla^{\prime}, \nabla, \nabla_{\text {trad }}\right)$ by (3) from the remark. Therefore, for differential operators $d_{\nabla^{\prime}}, d_{\nabla}, d_{\text {trad }}$ the condition (6) from Lemma 3.1 holds. Since the exterior multiplication

$$
\Lambda: \Lambda^{r} \mathfrak{g}^{*} \times \Lambda^{n-r} \mathfrak{g}^{*} \rightarrow \Lambda^{n} \mathfrak{g}^{*} \cong \mathbb{R}
$$

is nondegenerate then according to Lemma 3.1 the multiplication in cohomology

$$
H_{\nabla^{\prime}}^{r}(\mathfrak{g}) \times H_{\nabla}^{n-r}(\mathfrak{g}) \rightarrow H_{\text {trad }}^{n}(\mathfrak{g}) \cong \mathbb{R}
$$

is nondegenerate as well, i.e. in particular

$$
H_{\nabla^{\prime}}^{0}(\mathfrak{g}) \cong\left(H_{\nabla}^{n}(\mathfrak{g})\right)^{*}
$$

Immediately from the above reasoning we obtain the following theorems.

Theorem 3.4. The multiplication of reals is compatible with the representations $\left(0, \nabla_{\text {trad }}, \nabla_{\text {trad }}\right)$ and the induced cohomology pairing

$$
H^{n-r}(\mathfrak{g}) \times H_{\text {trad }}^{r}(\mathfrak{g}) \rightarrow H_{\text {trad }}^{n}(\mathfrak{g}) \cong \mathbb{R},
$$

is nondegenerate. In particular we obtain a noncanonical isomorphism

$$
H_{\text {trad }}^{r}(\mathfrak{g}) \cong\left(H_{\text {trad }}^{r}(\mathfrak{g})\right)^{*} \cong H^{n-r}(\mathfrak{g}) \text {. }
$$

Theorem 3.5. $\nabla_{\text {trad }}$ is the unique representation $\nabla$ for which $H_{\nabla}^{n}(\mathfrak{g}) \neq 0$.

Proof. For any representation $\nabla$ take $\nabla^{\prime}=\nabla_{\text {trad }}-\nabla$. By (2) from the remark above and isomorphism (3) we have

$$
H_{\nabla}^{n}(\mathfrak{g}) \cong H_{\nabla^{\prime}}^{0}(\mathfrak{g}) \neq 0 \Longleftrightarrow \nabla^{\prime}=0 \Longleftrightarrow \nabla=\nabla_{\text {trad }}
$$

\section{PAirings For GRADED Filtered DifFERENTIAL $\mathbb{R}$-VECTOR SPACES AND SPECTRAL SEQUENCES}

The aim of this chapter is to prove that for any pairing of graded regularly filtered differential $\mathbb{R}$-vector spaces, if the second terms of spectral sequences gives the nondegenerate pairing then the same holds for the cohomology algebras of the spaces. This holds without assumption that $\operatorname{dim} E_{2}$ is finite and generalizes the suitable theorem for graded filtered differential algebras [K-M].

Given three graded filtered differential $\mathbb{R}$-vector spaces

$$
\left({ }^{r} A=\bigoplus_{i \geq 0}{ }^{r} A^{i},{ }^{r} d,{ }^{r} A_{j}\right), \quad r=1,2,3,
$$

denote for shortness

$$
{ }^{r} H:=H\left({ }^{r} A,{ }^{r} d\right)
$$

Assume

$$
\text { : }{ }^{1} A \times{ }^{2} A \rightarrow{ }^{3} A
$$

preserves gradations and filtrations

$$
\begin{aligned}
& { }^{1} A^{s} \cdot{ }^{2} A^{t} \subset{ }^{3} A^{s+t}, \\
& { }^{1} A_{j} \cdot{ }^{2} A_{k} \subset{ }^{3} A_{j+k},
\end{aligned}
$$

and that the differentials ${ }^{r} d$ satisfy the compatibility condition

$$
{ }^{3} d(x \cdot y)={ }^{1} d x \cdot y+(-1)^{\operatorname{deg} x} x \cdot{ }^{2} d y .
$$


Clearly, there exists a multiplication of cohomology classes

$$
\cdot:{ }^{1} H^{j} \times{ }^{2} H^{k} \rightarrow{ }^{3} H^{j+k}, \quad([x],[y]) \mapsto[x \cdot y] .
$$

Let

$$
\left({ }^{r} E_{s}^{j, i},{ }^{r} d_{s}\right)
$$

be spectral sequences of graded filtered differential $\mathbb{R}$-vector spaces (4.1).

Lemma 4.1. (1)

$$
{ }^{1} Z_{s}^{j, i} \cdot{ }^{2} Z_{s}^{k, l} \subset{ }^{3} Z_{s}^{j+k, i+l}, \quad 0 \leq s \leq \infty
$$

$$
\begin{aligned}
{ }^{1} Z_{s}^{j, i} \cdot{ }^{2} D_{s-1}^{k, l}+{ }^{1} D_{s-1}^{j, k} \cdot{ }^{2} Z_{s}^{i, l} & \subset{ }^{3} Z_{s-1}^{j+k+1, i+l-1}+{ }^{3} D_{s-1}^{j+k, i+l}, \quad 0 \leq s<\infty, \\
{ }^{1} Z_{\infty}^{j, i} \cdot{ }^{2} D_{\infty}^{k, l}+{ }^{1} D_{\infty}^{j, i} \cdot{ }^{2} Z_{\infty}^{k, l} & \subset{ }^{3} D_{\infty}^{j+k, i+l}, \quad(s=\infty) .
\end{aligned}
$$

Proof. Straightforward calculations.

Conclusion 4.2. There exists a multiplication of s-terms of spectral sequences

$$
{ }^{1} E_{s}^{j, i} \times{ }^{2} E_{s}^{k, l} \rightarrow{ }^{3} E_{s}^{j+k . i+l},([x],[y]) \mapsto[x \cdot y], \quad 0 \leq s \leq \infty .
$$

The differentials ${ }^{1} d_{s},{ }^{2} d_{s},{ }^{3} d_{s}$ fulfils the compatibility condition with respect to the total gradation

$$
{ }^{3} d_{s}(x \cdot y)={ }^{1} d_{s} x \cdot y+(-1)^{\operatorname{total} \operatorname{deg} x} x \cdot{ }^{2} d_{s} y .
$$

There exists a multiplication of cohomology classes of $s$-terms

$$
H^{j, i}\left({ }^{1} E_{s},{ }^{1} d_{s}\right) \times H^{k, l}\left({ }^{2} E_{s},{ }^{2} d_{s}\right) \rightarrow H^{j+k, i+l}\left({ }^{3} E_{s},{ }^{3} d_{s}\right),([\tilde{x}],[\tilde{y}]) \mapsto[\tilde{x} \cdot \tilde{y}] .
$$

The linear isomorphisms of bigraded spaces

$$
\begin{aligned}
& { }^{r} \sigma_{s}:{ }^{r} E_{s+1} \rightarrow H\left({ }^{r} E_{s},{ }^{r} d_{s}\right), \\
& { }^{r} \sigma_{\infty}:{ }^{r} E_{\infty} \rightarrow E_{0}\left({ }^{r} H\right)
\end{aligned}
$$

conserve the multiplications

$$
\begin{aligned}
{ }^{3} \sigma_{s}([x] \cdot[y]) & ={ }^{1} \sigma_{s}[x] \cdot{ }^{2} \sigma_{s}[y], \\
{ }^{3} \sigma_{\infty}(\bar{x} \cdot \bar{y}) & ={ }^{1} \sigma_{\infty}(\bar{x}) \cdot{ }^{2} \sigma_{\infty}(\bar{y}) .
\end{aligned}
$$

Remark 4.3. For $s \geq i+2$ we consider the canonical epimorphisms

$$
{ }^{r} \gamma_{s}^{j, i}:{ }^{r} E_{s}^{j, i} \cong{ }^{r} Z_{\infty}^{j, i} /\left({ }^{r} Z_{\infty}^{j+1, i-1}+{ }^{r} D_{s-1}^{j, i}\right) \rightarrow{ }^{r} Z_{\infty}^{j, i} /\left({ }^{r} Z_{\infty}^{j+1, i-1}+{ }^{r} D_{\infty}^{j, i}\right) \cong{ }^{r} E_{\infty}^{j, i} .
$$

For $s \geq i+l+2$ the canonical epimorphisms ${ }^{1} \gamma_{s}^{j, i},{ }^{2} \gamma_{s}^{k, l},{ }^{3} \gamma_{s}^{j+k, i+l}$ are compatible with multiplications

$$
{ }^{3} \gamma_{s}^{j+k, i+l}([x] \cdot[y])={ }^{1} \gamma_{s}^{j, i}[x] \cdot{ }^{2} \gamma_{s}^{k, l}[y] .
$$

This implies that if spectral sequences $\left({ }^{r} E_{s},{ }^{r} d_{s}\right)$ collapse at the $\bar{m} t h$ term then the canonical isomorphisms ${ }^{r} \beta_{\bar{m}}:{ }^{r} E_{\bar{m}} \cong{ }^{r} E_{\infty}$, see [G-H-V, Vol.III. §1.1.2], conserve bigradations and are compatible with multiplications. We recall the construction of ${ }^{r} \beta_{\bar{m}}$. For arbitrary $(j, i)$ we select arbitrary $s \geq \max (\bar{m}, i+2)$ and put

$$
{ }^{r} \beta_{\bar{m}}^{j, i}:{ }^{r} E_{\bar{m}}^{j, i} \stackrel{r^{j} \sigma_{\bar{m}}^{j, i}}{\cong}{ }^{r} E_{\bar{m}+1}^{j, i} \cong \ldots \longleftarrow{ }^{r} E_{s}^{j, i} \stackrel{{ }^{r} \gamma_{s}^{j, i}}{\rightarrow}{ }^{r} E_{\infty}^{j, i}
$$

The following main result of this chapter generalizes Corollary 12 from [K-M]. 
Theorem 4.4. Given three graded filtered differential $\mathbb{R}$-vector spaces (4.1) and a pairing : : ${ }^{1} A \times{ }^{2} A \rightarrow{ }^{3} A$ satisfying (4.2), (4.3), (4.4), assume that the filtrations are regular in the sense ${ }^{r} A_{0}={ }^{r} A$ and that the second terms ${ }^{r} E_{2}^{j, i}$ live in the rectangular $0 \leq j \leq m, 0 \leq i \leq n$ and that ${ }^{3} E_{2}^{(m+n)}={ }^{3} E_{2}^{m, n} \cong \mathbb{R}$.

If the multiplication in the second terms

$$
\langle\cdot, \cdot\rangle_{2}:{ }^{1} E_{2}^{(j)} \times{ }^{2} E_{2}^{(m+n-j)} \rightarrow{ }^{3} E_{2}^{m, n} \cong \mathbb{R}
$$

is nondegenerate in the sense that

$$
{ }^{1} E_{2}^{(j)} \stackrel{\cong}{\longrightarrow}\left({ }^{2} E_{2}^{(m+n-j)}\right)^{*}, \quad x \mapsto\langle x, \cdot\rangle_{2},
$$

is a linear isomorphism, then

(a) ${ }^{3} H^{m+n} \cong \mathbb{R}$,

(b) ${ }^{r} H^{t}=0$ for $t>m+n$,

(c) the multiplication in cohomology classes

$$
\langle\cdot, \cdot\rangle_{H}:{ }^{1} H^{j} \times{ }^{2} H^{m+n-j} \rightarrow{ }^{3} H^{m+n} \cong \mathbb{R}
$$

is nondegenerate as well, i.e.

$$
{ }^{1} H^{j} \stackrel{\cong}{\longrightarrow}\left({ }^{2} H^{m+n-j}\right)^{*}, \quad[x] \mapsto\langle[x], \cdot\rangle_{H},
$$

is a linear isomorphism.

Proof. The terms ${ }^{r} E_{3},{ }^{r} E_{4}, \ldots,{ }^{r} E_{\infty}$ live also in the same rectangular $0 \leq j \leq m$, $0 \leq i \leq n$. The bidegree argument of the second differential operator ${ }^{3} d_{2}$ implies (compare with $[\mathrm{K}-\mathrm{M}]$ ) the condition ${ }^{3} d_{2}\left[{ }^{3} E_{2}^{(m+n-1)}\right]=0$. By the generalized ChernHirzebruch-Serre Lemma 3.1 we get ${ }^{3} E_{2}^{(m+n)}={ }^{3} E_{2}^{m, n} \cong \mathbb{R}$ and nondegeneracy of the multiplication for third terms. Proceeding inductively we get the same for all finite terms. The bidegree argument for the further differential operators ${ }^{r} d_{s}$ implies the colapsing of spectral sequences $\left({ }^{r} E_{s},{ }^{r} d_{s}\right)$, say at ${ }^{r} m>\max (m+1, n+2)$ places. Then ${ }^{3} E_{\infty}^{(m+n)}={ }^{3} E_{\infty}^{m, n} \cong \mathbb{R}$ so (a) holds because ${ }^{3} H^{m+n} \cong{ }^{3} E_{\infty}^{(m+n)} \cong \mathbb{R}$ and next, for $\bar{m} \geq \max \left({ }^{1} m,{ }^{2} m,{ }^{3} m\right.$ ) the canonical isomorphisms ${ }^{r} \beta_{\bar{m}}$ (see Remark 4.3) are compatible with multiplications. In consequence, the multiplication in the infinite terms

$$
\cdot:{ }^{1} E_{\infty}^{(j)} \times{ }^{2} E_{\infty}^{(m+n-j)} \rightarrow{ }^{3} E_{\infty}^{m, n} \cong \mathbb{R}
$$

is nondegenerate as well.

It remains to prove the nondegeneracy of the multiplication of cohomology classes (4.5). The spaces ${ }^{r} H$ possess a natural graded filtration ${ }^{r} H^{j, i}$, and thanks to the regularity of filtrations we have

$$
{ }^{r} H^{t}={ }^{r} H^{0, t} \supset{ }^{r} H^{1, t-1} \supset \ldots \supset{ }^{r} H^{t, 0} \supset 0
$$

and a noncanonical isomorphism

$$
{ }^{r} H^{t} \cong\left({ }^{r} H^{0, t} /{ }^{r} H^{1, t-1}\right) \oplus\left({ }^{r} H^{1, t-1} /{ }^{r} H^{2, t-2}\right) \oplus \ldots \oplus{ }^{r} H^{t, 0}=\bigoplus_{j+i=t} E_{0}^{j, i}\left({ }^{r} H\right) .
$$

Analogously to the proof of Theorem 11 from $[\mathrm{K}-\mathrm{M}]$ we assert that

$$
E_{0}^{(m+n)}\left({ }^{r} H\right)=E_{0}^{m, n}\left({ }^{r} H\right)={ }^{r} H^{m, n},
$$

and

$$
{ }^{r} H^{j, i}={ }^{r} H^{j+1, i-1} \text { for } j>m \text { or } i>n .
$$


Therefore by (4.8) ${ }^{r} H^{t}=0$ for $t>m+n$ which proves (b). As in [K-M] we check the rule:

- if ${ }^{1} \sigma_{\infty}^{j, i}(\bar{x})=[x]$ for $\bar{x} \in{ }^{1} E_{\infty}^{j, i}, x \in{ }^{1} H^{j, i}$, and if ${ }^{2} \sigma_{\infty}^{m-j, n-i}(\bar{y})=[y]$ for $\bar{y} \in{ }^{2} E_{\infty}^{m-j, n-i}, y \in{ }^{2} H^{m-j, n-i}$, then

$$
{ }^{3} \sigma_{\infty}^{m, n}(\bar{x} \cdot \bar{y})=[x] \cdot[y]=x \cdot y .
$$

We fix generators $\xi_{\infty} \in{ }^{3} E_{\infty}^{m, n}$ and $\xi_{H} \in{ }^{3} H^{m, n}$ in such a way that ${ }^{3} \sigma_{\infty}^{m, n}\left(\xi_{\infty}\right)=\xi_{H}$. Consider the pairings, see (4.6),

$$
\begin{gathered}
\langle\cdot, \cdot\rangle_{\infty}:{ }^{1} E_{\infty}^{(j)} \times{ }^{2} E_{\infty}^{(m+n-j)} \rightarrow \mathbb{R},\langle\bar{x}, \bar{y}\rangle_{\infty} \cdot \xi_{\infty}=\bar{x} \cdot \bar{y}, \\
\langle\cdot, \cdot\rangle_{H}:{ }^{1} H^{j} \times{ }^{2} H^{m+n-j} \rightarrow \mathbb{R},\langle x, y\rangle_{H} \cdot \xi_{H}=x \cdot y .
\end{gathered}
$$

By (4.10) we have

$$
\langle\bar{x}, \bar{y}\rangle_{\infty}=\langle x, y\rangle_{H}
$$

where ${ }^{1} \sigma_{\infty}^{j, i}(\bar{x})=[x]$ and ${ }^{2} \sigma_{\infty}^{m-j, n-i}(\bar{y})=[y]$. According to (4.6) the pairing $\langle\cdot, \cdot\rangle_{\infty}$ is nondegenerate, that is ${ }^{1} E_{\infty}^{(j)} \cong\left({ }^{2} E_{\infty}^{(m+n-j)}\right)^{*}$. Consider the induced linear mapping

$$
\kappa:{ }^{1} H^{j} \rightarrow\left({ }^{2} H^{m+n-j}\right)^{*}, \quad x \mapsto\langle x, \cdot\rangle_{H} .
$$

Similarly to $[\mathrm{K}-\mathrm{M}]$ we easily check the monomorphy of $\kappa$. It remains to check that $k$ is an epimorphism.

Take a linear function $0 \neq l:{ }^{2} H^{m+n-j} \rightarrow \mathbb{R}$ and consider the filtration (4.7) for $r=2$ and $t=m+n-j$. Let $V^{p} \subset{ }^{2} H^{p, m+n-j-p}$ be a subspace complementary to ${ }^{2} H^{p+1, m+n-j-p-1}, p=0,1, \ldots, m+n-j$ and

$$
\psi^{p}: V^{p} \rightarrow E_{0}^{p, m+n-j-p}\left({ }^{2} H\right), \quad x \mapsto[x],
$$

the induced isomorphism. Put

$$
\psi=\sum_{p} \psi^{p}:{ }^{2} H^{m+n-j}=\bigoplus_{p} V^{p} \cong \bigoplus_{p} E_{0}^{p, m+n-j-p}\left({ }^{2} H\right) .
$$

The composition $l \circ \psi^{-1} \in\left(\bigoplus_{p} E_{0}^{p, m+n-j-p}\left({ }^{2} H\right)\right)^{*}$ determines a family of linear functions $l_{0}^{p} \in\left(E_{0}^{p, m+n-j-p}\left({ }^{2} H\right)\right)^{*}$. Define

$$
I_{l}=\left\{p ; l_{0}^{p} \neq 0\right\} .
$$

For each $p \in I_{l}$ we define - through isomorphisms

$$
{ }^{2} \sigma_{\infty}^{p, m+n-j-p}:{ }^{2} E_{\infty}^{p, m+n-j-p} \stackrel{\cong}{\longrightarrow} E_{0}^{p, m+n-j-p}\left({ }^{2} H\right)
$$

- a linear nonzero functions

$$
l_{\infty}^{p} \in\left({ }^{2} E_{\infty}^{p, m+n-j-p}\right)^{*}, l_{\infty}^{p}=l_{0}^{p} \circ{ }^{2} \sigma_{\infty}^{p, m+n-j-p} .
$$

The nondegenerate pairing $\langle\cdot, \cdot\rangle_{\infty}:{ }^{1} E_{\infty}^{m-p, p+j-m} \times{ }^{2} E_{\infty}^{p, m+n-j-p} \rightarrow \mathbb{R}$ determines an element $0 \neq \bar{x}^{m-p} \in{ }^{1} E_{\infty}^{m-p, p+j-m}$ such that $\left\langle\bar{x}^{m-p}, \cdot\right\rangle_{\infty}=l_{\infty}^{p} \in\left({ }^{2} E_{\infty}^{p, m+n-j-p}\right)^{*}$. Let ${ }^{1} \sigma_{\infty}^{m-p, p+j-m}\left(\bar{x}^{m-p}\right)=\left[x^{m-p}\right] \in E_{0}^{m-p, p+j-m}\left({ }^{1} H\right)$, where

$$
x^{m-p} \in{ }^{1} H^{m-p, p+j-m} \text { and } x^{m-p} \notin{ }^{1} H^{m-p+1, p+j-m-1} .
$$

Put

$$
x:=\sum_{p \in I_{l}} x^{m-p} .
$$


We prove the equality

$$
\kappa(x)=\langle x, \cdot\rangle_{H}=l \in\left({ }^{2} H^{m+n-j}\right)^{*} .
$$

Since ${ }^{2} H^{m+n-j}=\bigoplus_{p} V^{p}$, we need only to prove $\kappa(x)\left(y^{p}\right)=\langle x, \cdot\rangle_{H}\left(y^{p}\right)=l\left(y^{p}\right)$ for $y^{p} \in V^{p} \subset{ }^{2} H^{p, m+n-j-p}$. If $p \notin I_{l}$, i.e. $l_{0}^{p}=0$, then $l\left(y^{p}\right)=0$ and for all $p^{\prime} \in I_{l}$ by (4.9) and (4.10)

$$
\left\langle x^{m-p^{\prime}}, y^{p}\right\rangle_{H} \cdot \xi_{H}=x^{m-p^{\prime}} \cdot y^{p}=\left[x^{m-p^{\prime}}\right] \cdot\left[y^{p}\right] \in E_{0}^{m-p+p^{\prime}, n+p-p^{\prime}}\left({ }^{3} H\right)=0 .
$$

If $p \in I_{l}$ and $0 \neq y^{p} \in V^{p}$ then

$$
\begin{aligned}
l\left(y^{p}\right) & =\left(l \mid V^{p}\right) \circ\left(\psi^{p}\right)^{-1}[y]=l_{0}^{p}\left[y^{p}\right]=l_{\infty}^{p} \circ\left({ }^{2} \sigma_{\infty}^{p, m+n-j-p}\right)^{-1}\left(\left[y^{p}\right]\right) \\
& =\left\langle\bar{x}^{m-p},\left({ }^{2} \sigma_{\infty}^{p, m+n-j-p}\right)^{-1}\left(\left[y^{p}\right]\right)\right\rangle_{\infty} \\
& \stackrel{(4.11)}{=}\left\langle x^{m-p}, y^{p}\right\rangle_{H} \\
& =\left\langle x, y^{p}\right\rangle_{H} .
\end{aligned}
$$

The last equation holds because for $p^{\prime} \neq p, p^{\prime} \in I_{l}$, we have

$$
0 \neq\left[x^{m-p^{\prime}}\right] \in E_{0}^{m-p^{\prime}, p^{\prime}+j-m}\left({ }^{1} H\right)
$$

and by (4.9) and (4.10)

$$
x^{m-p^{\prime}} \cdot y^{p}=\left[x^{m-p^{\prime}}\right] \cdot\left[y^{p}\right] \in E_{0}^{m-p^{\prime}, p^{\prime}+j-m}\left({ }^{3} H\right)=0 .
$$

\section{Hochschild-Serre Filtration And the SPeCtral Sequence For TRANSITIVE LIE ALGEBROIDS}

We fix a transitive Lie algebroid $A=\left(A, \llbracket \cdot, \cdot \rrbracket, \#_{A}\right)$ with the Atiyah sequence $0 \rightarrow \boldsymbol{g} \hookrightarrow A \stackrel{\#_{A}}{\rightarrow} T M \rightarrow 0$ and a representation $\nabla: A \rightarrow A(\xi)$ of a Lie algebroid $A$ on a vector bundle $\xi . \nabla$ is a homomorphism of Lie algebroids, then $\nabla$ induces a homomorphism of vector bundles $\nabla^{+}: \boldsymbol{g} \rightarrow$ End $(\xi)$

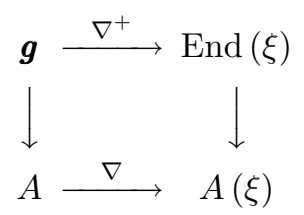

and $\nabla_{x}^{+}: \boldsymbol{g}_{x} \rightarrow$ End $\left(\xi_{x}\right)$ is a representation of the isotropy Lie algebra $\boldsymbol{g}_{x}$ in the vector space $\xi_{x}$. We will consider the pair of $\mathbb{R}$-Lie algebras $(\mathfrak{g}, \mathfrak{k})$ where

$$
\mathfrak{g}=\Gamma(A), \mathfrak{k}=\Gamma(\boldsymbol{g}) .
$$

Below, the elements of $\mathfrak{g}$ will be denoted by $\gamma, \gamma_{1}, \gamma_{2}, \ldots$ while elements of $\mathfrak{k}$ by $\sigma, \sigma_{1}, \sigma_{2}, \ldots$. Of course, $\mathfrak{k}$ is an ideal of $\mathfrak{g}$ (actually, $\mathfrak{k}$ is $C^{\infty}(M)$-Lie algebra but it is not interesting here). The space $\Gamma(\xi)$ is a $\mathfrak{g}$-modul with respect to the induced representation denoted by the same letter $\nabla: \Gamma(A) \rightarrow C D O(\xi) \subset L_{\Gamma(\xi)}$. Following Hochschild-Serre [H-S] we can consider a graded cochain group of $\mathbb{R}$-linear alternating functions

$$
A_{\mathbb{R}}=\bigoplus_{i \geq 0} A^{i}, \quad A^{i}=C^{i}(\mathfrak{g}, \Gamma(\xi))
$$


with the $\mathbb{R}$-differential operator of degree 1

$$
d_{\nabla}: C^{i}(\mathfrak{g}, \Gamma(\xi)) \rightarrow C^{i+1}(\mathfrak{g}, \Gamma(\xi))
$$

defined by the standard formula

$$
\left(d_{\nabla} f\right)\left(\gamma_{0}, \ldots, \gamma_{t}\right)=\sum_{i}(-1)^{i} \nabla_{\gamma_{i}}\left(f\left(\gamma_{0}, \ldots \hat{\imath} \ldots, \gamma_{t}\right)\right)+\sum_{i<j}(-1)^{i+j} f\left(\llbracket \gamma_{i}, \gamma_{j} \rrbracket, \ldots \hat{\imath} \ldots \hat{\jmath} \ldots\right) .
$$

For the trivial representation $\partial_{A}: A \rightarrow A(M \times \mathbb{R}),\left(\partial_{A}\right)_{\gamma}(f)=\partial_{\#(\gamma)}(f)$, this operator is denoted by $d_{A}$. Clearly, for a real alternating $t$-cocycle $\varphi$ and $\sigma \in \Gamma(\xi)$ we get

$$
d_{\nabla}(\varphi \otimes \sigma)=d_{A} \varphi \otimes \sigma+(-1)^{t} \varphi \wedge d_{\nabla} \sigma .
$$

In the space $\bigoplus_{t \geq 0} A^{t}$ we have the Hochschild-Serre filtration $A_{j} \subset A_{\mathbb{R}}$ as follows: $A_{j}=A_{\mathbb{R}}$ for $j \leq 0$. If $j>0, A_{j}=\bigoplus_{t \geq j} A_{j}^{t}, A_{j}^{t}=A_{j} \cap A^{t}$, where $\mathrm{A}_{j}^{t}$ consists of all those $t$-cochains $f$ for which $f\left(\gamma_{1}, \ldots, \gamma_{t}\right)=0$ whenever $t-j+1$ of the arguments $\gamma_{i}$ belongs to $\mathfrak{k}$. In this way we have obtained a graded filtered differential $\mathbb{R}$-vector space

$$
\left(A_{\mathbb{R}}=\bigoplus_{t \geq 0} A^{t}, d_{\nabla}, A_{j}\right)
$$

and we can use its spectral sequence

$$
\left(E_{s}^{j, i}, d_{s}\right)
$$

Following K.C.M.Mackenzie [M] (see also V.Itskov, M.Karashev, and Y.Vorobjev [I-K-V]) we will consider the $C^{\infty}(M)$-submodule of $C^{\infty}(M)$-linear altarnating cochains with values in the vector bundle $\xi$ (i.e. $A$-differential $\xi$-valued forms)

$$
\Omega^{t}(A, \xi) \subset C^{t}(\mathfrak{g}, \Gamma(\xi))
$$

and the induced filtration

$$
\Omega_{j}=\Omega_{j}(A, \xi)=A_{j} \cap \Omega(A, \xi)
$$

of $C^{\infty}(M)$-modules. The differential $d_{\nabla}$ of a $C^{\infty}(M)$-cochain is a $C^{\infty}(M)$-cochain, so we get $d_{A, \nabla}: \Omega(A, \xi) \rightarrow \Omega(A, \xi)$. We obtain in this way a graded filtered differential space

$$
\left(\Omega(A, \xi)=\bigoplus_{t} \Omega^{t}(A, \xi), d_{A, \nabla}, \Omega_{j}\right)
$$

and its spectral sequence

$$
\left(E_{A, s}^{j, i}, d_{A, \nabla, s}\right) .
$$

Now we consider as well a submodule of $C^{\infty}(M)$-linear altarnating cochains with compact support $\Omega_{c}^{t}(A, \xi) \subset \Omega^{t}(A, \xi)$ and the corresponding filtration

$$
\Omega_{c, j}=\Omega_{j} \cap \Omega_{c}(A, \xi)
$$

of $C^{\infty}(M)$-modules. Since supp $d_{\nabla} f \subset \operatorname{supp} f$ then we obtain

$$
d_{A_{c}, \nabla}: \Omega_{c}(A, \xi) \rightarrow \Omega_{c}(A, \xi)
$$

and we get a graded filtered differential space with compact support

$$
\left(\Omega_{c}(A, \xi)=\bigoplus_{t} \Omega_{c}^{t}(A, \xi), d_{A_{c}, \nabla}, \Omega_{c, j}\right)
$$


and its spectral sequence

$$
\left(E_{A_{c}, s}^{j, i}, d_{A_{c}, \nabla, s}\right) \text {. }
$$

Sometimes we can deduce directly properties of the last two spectral sequences (5.4), (5.6) from the suitable properties of (5.2), see [H-S], denoted further by $A_{\mathbb{R}}$, sometimes we must use some additional observations.

Lemma 5.1. The homomorphisms $\rho_{0} \rho_{c, 0}$ in the sequence

$$
E_{A_{c}, 0}^{j, i}=\Omega_{c, s}^{j+i} / \Omega_{c, s+1}^{j+i} \stackrel{\rho_{c, 0}}{\longmapsto} \Omega_{s}^{j+i} / \Omega_{s+1}^{j+i}=E_{A, 0}^{j, i} \stackrel{\rho_{0}}{\longmapsto} A_{s}^{j+i} / A_{j+1}^{j+i}=E_{0}^{j, i}
$$

are monomorphisms. For differentials $d_{A_{c}, \nabla}, d_{A, \nabla}, d_{0}$ the following diagram is commutative

$$
\begin{array}{ccccc}
E_{A_{c}, 0}^{j, i} & \stackrel{\rho_{c, 0}}{\longrightarrow} & E_{A, 0}^{j, i} \stackrel{\rho_{0}}{\longrightarrow} & E_{0}^{j, i} \\
\quad d_{A_{c}, \nabla, 0}^{j, i} & & d_{A, \nabla, 0}^{j, i} & & \downarrow d_{0}^{j, i} \\
E_{A_{c}, 0}^{j, i+1} & \stackrel{\rho_{c, 0}}{\longrightarrow} & E_{A, 0}^{j, i+1} & \stackrel{\rho_{0}}{\longrightarrow} & E_{0}^{j, i+1} .
\end{array}
$$

From

- $A_{\mathbb{R}}$ For R-cochains there exists an isomorphism

$$
a^{j, i}: E_{0}^{j, i} \rightarrow C^{j}\left(\mathfrak{g} / \mathfrak{k}, C^{i}(\mathfrak{k}, \Gamma(\xi))\right)
$$

such that

$$
a^{j, i}[f]\left(\left[\gamma_{1}\right], \ldots,\left[\gamma_{j}\right]\right)\left(\sigma_{1}, \ldots, \sigma_{i}\right)=f\left(\sigma_{1}, \ldots, \sigma_{i}, \gamma_{1}, \ldots, \gamma_{j}\right), .
$$

we can easily obtain the following

Conclusion 5.2. The homomorphisms

$$
\begin{gathered}
a_{A}^{j, i}: E_{A, 0}^{j, i} \rightarrow \Omega^{j}\left(M, \Lambda^{i} \boldsymbol{g}^{*} \otimes \xi\right) \\
a_{A_{c}}^{j, i}: E_{A_{c}, 0}^{j, i} \rightarrow \Omega_{c}^{j}\left(M, \Lambda^{i} \boldsymbol{g}^{*} \otimes \xi\right)
\end{gathered}
$$

defined by the formula

$$
a_{A}^{j, i}[f]\left(X_{1}, \ldots, X_{j}\right)\left(\sigma_{1}, \ldots, \sigma_{i}\right)=f\left(\sigma_{1}, \ldots, \sigma_{i}, \lambda X_{1}, \ldots, \lambda X_{j}\right),
$$

$X_{j^{\prime}} \in \mathfrak{X}(M), \sigma_{i^{\prime}} \in \mathfrak{k},\left(a_{A_{c}}^{j, i}\right.$ defined by the identical formula) where $\lambda: T M \rightarrow A$ is an arbitrary connection, are correctly defined linear isomorphisms of $C^{\infty}(M)$ modules.

Proof. Monomorphy of $a_{A}^{j, i}$ and $a_{A_{c}}^{j, i}$ follows from the commutativity of the diagram

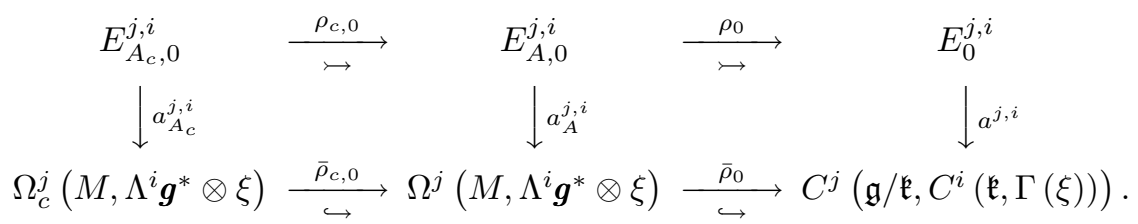

To prove that $a_{A}^{j, i}$ is an epimorphism it is sufficient to check that if $a^{j, i}[f]$ is a $C^{\infty}(M)$-linear cochain, i.e. $a^{j, i}[f]=\bar{\rho}_{0}(\bar{f})$ for some $\bar{f} \in \Omega^{j}\left(M, \Lambda^{i} \boldsymbol{g}^{*} \otimes \xi\right)$, i.e. $\bar{f}\left(\#_{A}\left(\gamma_{1}\right), \ldots, \#_{A}\left(\gamma_{j}\right)\right)\left(\sigma_{1}, \ldots, \sigma_{i}\right)=f\left(\sigma_{1}, \ldots, \sigma_{i}, \gamma_{1}, \ldots, \gamma_{j}\right)$, then there exists a representative $f^{\prime} \in[f] \in E_{A, 0}^{j, i}$ which is $C^{\infty}(M)$-linear cochain such that $a_{A}^{j, i}\left[f^{\prime}\right]=\bar{f}$. 
To this end take a connection form $\omega_{0}: A \rightarrow \boldsymbol{g}$ coresponding to $\lambda$ and put $f^{\prime}\left(\gamma_{1}^{\prime}, \ldots, \gamma_{j}^{\prime}, \gamma_{1}, \ldots, \gamma_{i}\right)=f\left(\omega_{0}\left(\gamma_{1}^{\prime}\right), \ldots, \omega_{0}\left(\gamma_{j}^{\prime}\right), \gamma_{1}, \ldots, \gamma_{i}\right)$. Then $f^{\prime}$ fulfils the desired conditions.

- $A_{\mathbb{R}}$ Through isomorphism $a^{j, i}$ the differential $d_{0}^{j, i}$ becomes a differentiation of values with respect to the differential

$$
d_{\nabla \circ \iota}: C^{i}(\mathfrak{k}, \Gamma(\xi)) \rightarrow C^{i+1}(\mathfrak{k}, \Gamma(\xi))
$$

$(\iota: \mathfrak{k} \hookrightarrow \mathfrak{g}$, is the inclusion),

$$
\begin{aligned}
\tilde{d}_{\nabla \circ \iota}: C^{j}\left(\mathfrak{g} / \mathfrak{k}, C^{i}(\mathfrak{k}, \Gamma(\xi))\right) & \rightarrow C^{j}\left(\mathfrak{g} / \mathfrak{k}, C^{i+1}(\mathfrak{k}, \Gamma(\xi))\right), \\
\tilde{d}_{\nabla \circ \iota}(f)\left(\left[\gamma_{1}\right], \ldots,\left[\gamma_{j}\right]\right) & =d_{\nabla \circ \iota}\left(f\left(\left[\gamma_{1}\right], \ldots,\left[\gamma_{j}\right]\right)\right) .
\end{aligned}
$$

In conclusion, the differentials $d_{A, \nabla, 0}^{j, i}$ and $d_{A_{c}, \nabla, 0}^{j, i}$ becomes (through the isomorphisms $a_{A}^{j, i}$ and $a_{A_{c}}^{j, i}$ ) differentials of values with respect to

$$
d_{\nabla^{+}}: \Lambda^{i} \boldsymbol{g}^{*} \otimes \xi \rightarrow \Lambda^{i+1} \boldsymbol{g}^{*} \otimes \xi
$$

namely

$$
\begin{aligned}
\tilde{d}_{\nabla^{+}}: \Omega^{j}\left(M, \Lambda^{i} \boldsymbol{g}^{*} \otimes \xi\right) & \rightarrow \Omega^{j}\left(M, \Lambda^{i+1} \boldsymbol{g}^{*} \otimes \xi\right), \\
\tilde{d}_{\nabla^{+}}(f)\left(X_{1}, \ldots, X_{j}\right) & =d_{\nabla^{+}}\left(f\left(X_{1}, \ldots, X_{j}\right)\right) .
\end{aligned}
$$

Analogously we obtain a differential $\tilde{d}_{c, \nabla^{+}}$for compact supports.

Remark 5.3. According to K.Mackenzie [M, Th.2.5, p.201] the homomorphisms $d_{\nabla^{+}}^{i}: \Lambda^{i} \boldsymbol{g}^{*} \otimes \xi \rightarrow \Lambda^{i+1} \boldsymbol{g}^{*} \otimes \xi$ are locally of constant rank, and consequently, there are well-defined vector bundles $Z^{i}=\operatorname{ker} d_{\nabla^{+}}^{i}, B^{i}=\operatorname{Im} d_{\nabla^{+}}^{i-1}$ and $H^{i}(\boldsymbol{g}, \xi)=Z^{i} / B^{i}$ such that $\Gamma\left(H^{i}(\boldsymbol{g}, \xi)\right)=H^{i}\left(\Gamma\left(\Lambda \boldsymbol{g}^{*} \otimes \xi\right), d_{\nabla^{+}}\right)$. Clearly,

$$
H^{i}(\boldsymbol{g}, \xi)_{x}=H^{i}\left(\Lambda \boldsymbol{g}_{x}^{*} \otimes \xi_{x}, d_{\nabla_{x}^{+}}\right) \text {. }
$$

Therefore

$$
\begin{aligned}
H\left(\Omega^{j}\left(M, \Lambda^{i} \boldsymbol{g}^{*} \otimes \xi\right), \tilde{d}_{\nabla^{+}}\right) & \cong \Omega^{j}\left(M, H_{\nabla^{+}}^{i}(\boldsymbol{g}, \xi)\right), \\
H\left(\Omega_{c}^{j}\left(M, \Lambda^{i} \boldsymbol{g}^{*} \otimes \xi\right), \tilde{d}_{c, \nabla^{+}}\right) & \cong \Omega_{c}^{j}\left(M, H_{\nabla^{+}}^{i}(\boldsymbol{g}, \xi)\right) .
\end{aligned}
$$

From the above we obtain isomorphisms of $C^{\infty}(M)$-modules

$$
\begin{aligned}
& \left(a_{A}^{j, i}\right)_{\#}: H^{i}\left(E_{A, 0}^{j, *}, d_{A, \nabla, 0}^{j, *}\right) \stackrel{\cong}{\rightrightarrows} \Omega^{j}\left(M, \Lambda^{i} \boldsymbol{g}^{*} \otimes \xi\right), \\
& \left(a_{A_{c}}^{j, i}\right)_{\#}: H^{i}\left(E_{A_{c}, 0}^{j, *}, d_{A_{c}, \nabla, 0}^{j, *}\right) \cong \Omega_{c}^{j}\left(M, \Lambda^{i} \boldsymbol{g}^{*} \otimes \xi\right) .
\end{aligned}
$$

Now we pass to consideration of the modules $Z_{s}, D_{s}, E_{s}$ and $Z_{A, s}, D_{A, s}, E_{A, s}$ and $Z_{A_{c}, s}, D_{A_{c}, s}, E_{A_{c}, s}$ for three spectral sequences for graded, filtered, differential spaces (5.1), (5.3), (5.5), respectively. Immediately by definitions we get

Lemma 5.4. (1) $Z_{A, s}^{j}=Z_{s}^{j} \cap \Omega(A, \xi)$, (2) $D_{A, s}^{j}=D_{s}^{j} \cap \Omega(A, \xi)$, (3) $Z_{A_{c}, s}^{j}=$ $Z_{A, s}^{j} \cap \Omega_{c}(A, \xi)$.

Fix an auxiliary a connection $\lambda: T M \rightarrow A$ and for $f \in Z_{A, 1}^{j, i} \subset \Omega^{j+i}(A, \xi)$ we define

$$
\bar{f}_{j} \in \Omega^{j}\left(M, \Lambda^{i} \boldsymbol{g}^{*} \otimes \xi\right)
$$


by the formula

$$
\begin{aligned}
\bar{f}_{j}\left(X_{1}, \ldots, X_{j}\right)\left(\sigma_{1}, \ldots, \sigma_{i}\right) & =f\left(\lambda X_{1}, \ldots, \lambda X_{j}, \sigma_{1}, \ldots, \sigma_{i}\right) \\
& =(-1)^{j i} f\left(\sigma_{1}, \ldots, \sigma_{i}, \lambda X_{1}, \ldots, \lambda X_{j}\right) .
\end{aligned}
$$

Lemma 5.5. If $f \in Z_{A, 1}^{j, i}$ then $\bar{f}_{j}\left(X_{1}, \ldots, X_{j}\right) \in \Gamma\left(\Lambda^{i} \boldsymbol{g}^{*} \otimes \xi\right)$ is a $d_{\nabla^{+}-\text {cocycle, }}$ $d_{\nabla^{+}}\left(\bar{f}_{j}\left(X_{1}, \ldots, X_{j}\right)\right)=0$, independent of the choice of $\lambda$.

Proof. For $f \in Z_{A, 1}^{j, i} \subset \Omega_{j}^{j+i}(A, \xi) \subset A_{s}^{j+i}$ we take a cochain $f_{j} \in C^{j}\left(\mathfrak{g}, C^{i}(\mathfrak{g}, \Gamma(\xi))\right)$ defined by $f_{j}\left(\gamma_{1},,,, \gamma_{j}\right)\left(\gamma_{1}^{\prime}, \ldots, \gamma_{i}^{\prime}\right)=f\left(\gamma_{1},,,, \gamma_{j}, \gamma_{1}^{\prime}, \ldots, \gamma_{i}^{\prime}\right)$, see [H-S]. From the equalities

$$
Z_{A, 1}^{j, i}=\left\{f \in \Omega_{j}^{j+i} ; d_{\nabla} f \in \Omega_{j+1}^{j+i+1}\right\}=Z_{1}^{j, i} \cap \Omega^{j+i}(A, \xi)
$$

we get (see $[\mathrm{H}-\mathrm{S}])$ that $\iota_{j}^{*}\left(f_{j}\left(\gamma_{1},,,, \gamma_{j}\right)\right) \in C^{j}(\mathfrak{k}, \Gamma(\xi))$, where

$$
\iota_{j}^{*}: C^{j}(\mathfrak{g}, \Gamma(\xi)) \rightarrow C^{j}(\mathfrak{k}, \Gamma(\xi)), \quad \iota_{j}^{*}(g)=g \mid \mathfrak{k} \times \ldots \times \mathfrak{k},
$$

is a $\left(\nabla \circ \iota: \mathfrak{k} \rightarrow L_{\Gamma(\xi)}\right)$-cocycle and that this cocycle depends only on the equivalence class $\left[\gamma_{j^{\prime}}\right] \in \mathfrak{g} / \mathfrak{k} \cong \mathfrak{X}(M)$, i.e. on the anchors of the elements $\gamma_{j^{\prime}}$, i.e. on $\#_{A}\left(\gamma_{j^{\prime}}\right)$. But $\iota_{j}^{*}\left(f_{j}\left(\gamma_{1},,,, \gamma_{j}\right)\right)$ is $C^{\infty}(M)$-linear $\iota_{j}^{*}\left(f_{j}\left(\gamma_{1},,,, \gamma_{j}\right)\right) \in \Gamma\left(\Lambda^{i} \boldsymbol{g}^{*} \otimes \xi\right)$ therefore the condition $d_{\nabla \circ \iota}\left(\iota_{j}^{*}\left(f_{j}\left(\gamma_{1},,,, \gamma_{j}\right)\right)\right)=0$ is equivalent to

$$
d_{\nabla^{+}}\left(\iota_{j}^{*}\left(f_{j}\left(\gamma_{1},,,, \gamma_{j}\right)\right)\right)=0 .
$$

The equality $\iota_{j}^{*}\left(f_{j}\left(\gamma_{1},,,, \gamma_{j}\right)\right)=\bar{f}_{j}\left(\#_{A}\left(\gamma_{1}\right), \ldots, \#_{A}\left(\gamma_{j}\right)\right)$ proves the lemma.

We recall that

$$
\begin{aligned}
E_{A, 1}^{j, i} & =Z_{A, 1}^{j, i} /\left(Z_{A, 0}^{j+1, i-1}+D_{A, 0}^{j, i}\right) \\
& =\left\{f \in \Omega_{j}^{j+i}(A, \xi) ; d_{\nabla} f \in \Omega_{j+1}^{j+i+1}\right\} /\left(\Omega_{j+1}^{j+i}+d_{\nabla}\left[\Omega_{j}^{j+i-1}\right]\right)
\end{aligned}
$$

and analogously for $E_{A_{c}, 1}^{j, i}$.

Lemma 5.6. The homomorphisms

$$
\begin{gathered}
\Psi_{A, 1}: E_{A, 1}^{j, i} \rightarrow \Omega^{j}\left(M, H_{\nabla^{+}}^{i}(\boldsymbol{g}, \xi)\right), \quad[f] \mapsto(-1)^{j i}\left[\bar{f}_{j}\right], \\
\Psi_{A_{c}, 1}: E_{A_{c}, 1}^{j, i} \rightarrow \Omega_{c}^{j}\left(M, H_{\nabla^{+}}^{i}(\boldsymbol{g}, \xi)\right), \quad[f] \mapsto(-1)^{j i}\left[\bar{f}_{j}\right],
\end{gathered}
$$

are isomorphisms of $C^{\infty}(M)$-modules.

Proof. Clearly, we need to notice only that $\Psi_{A, 1}$ is a composition of isomorphisms

$$
\Psi_{A, 1}: E_{A, 1}^{j, i} \stackrel{\sigma_{A, 0}^{j, i}}{\cong} H^{i}\left(E_{A, 0}^{j, *}, d_{A, 0}^{j, *}\right) \stackrel{\left(a_{A}^{j, i}\right)_{\#}}{\cong} \Omega^{j}\left(M, H_{\nabla^{+}}^{i}(\boldsymbol{g}, \xi)\right)
$$

and analogously for compact supports.

From the above lemmas we see that the canonical homomorphism $E_{A_{c}, 1}^{j, i} \rightarrow E_{A, 1}^{j, i}$ is a monomorphism.

- $A_{\mathbb{R}}$ There exists a representation [precisely, a Lie derivation]

$$
\mathcal{L}^{i}: \mathfrak{g} \rightarrow L_{C^{i}(\mathfrak{k}, \Gamma(\xi))}
$$

defined by the formula

$\left(\mathcal{L}_{\gamma}^{i} f\right)\left(\sigma_{1}, \ldots, \sigma_{i}\right)=\nabla_{\gamma}\left(f\left(\sigma_{1}, \ldots, \sigma_{i}\right)\right)-\sum_{t} f\left(\sigma_{1}, \ldots, \llbracket \gamma, \sigma_{t} \rrbracket, \ldots, \sigma_{i}\right)$. 
$\mathcal{L}_{\gamma}^{i}$ commutes with $\mathbb{R}$-differential operator $d_{\nabla \circ \iota}$, see (5.8), induces a representation in cohomology

$$
\mathcal{L}^{\#, i}: \mathfrak{g} \rightarrow L_{H_{\nabla \circ \iota}^{i}(\mathfrak{k}, \Gamma(\xi))}
$$

and $\mathfrak{k} \subset \operatorname{ker} \mathcal{L}^{\#, i}$ (because $\mathcal{L}_{\sigma}^{i} f=d_{\nabla \circ \iota}\left(\iota_{\sigma} f\right)$ if $f$ is a $d_{\nabla \circ \iota}$-cocycle.). It produces a representation

$$
\left[\mathcal{L}^{\#, i}\right]: \mathfrak{g} / \mathfrak{k} \rightarrow L_{H_{\nabla \circ \iota}^{i}(\mathfrak{k}, \Gamma(\xi))} .
$$

Noticing that a Lie derivation of a $C^{\infty}(M)$-linear cochain is $C^{\infty}(M)$-linear too, we can pass to $\Gamma\left(\Lambda^{i} \boldsymbol{g}^{*} \otimes \xi\right)$. Additionally we observe that $\mathcal{L}_{\gamma}^{i}: \Gamma\left(\Lambda^{i} \boldsymbol{g}^{*} \otimes \xi\right) \rightarrow$ $\Gamma\left(\Lambda^{i} \boldsymbol{g}^{*} \otimes \xi\right)$ is a covariant derivative operator with the anchor $\#_{A}(\gamma)$ and $\mathcal{L}_{\gamma}^{i}$ is $C^{\infty}(M)$-linear with respect to $\gamma$. In conclusion we obtain a representation of the Lie algebroid $A$ in the vector bundle $\Lambda^{i} \boldsymbol{g}^{*} \otimes \xi$

$$
\mathcal{L}_{A}^{i}: A \rightarrow A\left(\Lambda^{i} \boldsymbol{g}^{*} \otimes \xi\right) .
$$

Lemma 5.7. The representation $\mathcal{L}_{A}^{i}$ coincides with the adjoint representaion of $A$ in $\Lambda^{i} \boldsymbol{g}^{*}$ cross $\nabla, \mathcal{L}_{A}^{i}=a d_{A} \otimes \nabla$.

Proof. The adjoint representation $a d_{A}: A \rightarrow A(\boldsymbol{g}), a d_{A}(\gamma)(\sigma)=\llbracket \gamma, \sigma \rrbracket$, induces the one in the associated bundle $\Lambda^{i} \boldsymbol{g}^{*}$ (denoted also $a d_{A}$ ) and its tensor product with $\nabla$ is just equal to $\mathcal{L}_{A}^{i}$.

The representation $\mathcal{L}_{A}^{i}$ induces the one in cohomology

$$
\mathcal{L}_{A}^{\#, i}: A \rightarrow A\left(H_{\nabla^{+}}^{i}(\boldsymbol{g}, \xi)\right)
$$

such that $\boldsymbol{g} \subset \operatorname{ker} \mathcal{L}_{A}^{\#, i}$ (indeed, $\left(\mathcal{L}_{A}^{i}\right)_{\sigma}(f)=d_{\nabla^{+}}\left(\iota_{\sigma} f\right)$ for a $d_{\nabla^{+}}$-cocycle $f$ ). Therefore, we obtain a flat covariant derivative

$$
\nabla^{i}: M \rightarrow A\left(H_{\nabla^{+}}^{i}(\boldsymbol{g}, \xi)\right)
$$

by the formula

$$
\nabla_{X}^{i}([f])=\left(\mathcal{L}_{A}^{\#, i}\right)_{\lambda X}([f])=\left[\left(\mathcal{L}_{A}^{i}\right)_{\lambda X}(f)\right]
$$

where for a $d_{\nabla^{+}-\text {cocycle } f \in \Lambda^{i} \boldsymbol{g}^{*} \otimes \xi}$

$$
\left(\mathcal{L}_{A}^{i}\right)_{\lambda X}(f)\left(\sigma_{1}, \ldots, \sigma_{i}\right)=\nabla_{\lambda X}\left(f\left(\sigma_{1}, \ldots, \sigma_{i}\right)\right)-\sum_{t} f\left(\sigma_{1}, \ldots, \llbracket \lambda X, \sigma_{t} \rrbracket, \ldots, \sigma_{i}\right) .
$$

Remark 5.8. For the trivial representation $\nabla=\partial_{A}$ we get a flat structure in the cohomology bundle $H^{i}(\boldsymbol{g})$. If the structure Lie algebras $\boldsymbol{g}_{x}$ are unimodular then $H^{n}(\boldsymbol{g})=\Lambda^{n} \boldsymbol{g}^{*}$ and the induced flat covariant derivative $\partial_{A}^{n}: M \rightarrow A\left(\Lambda^{n} \boldsymbol{g}^{*}\right)$ is defined by

$$
\left(\left(\partial_{A}^{n}\right)_{X} f\right)\left(\sigma_{1}, \ldots, \sigma_{n}\right)=X\left(f\left(\sigma_{1}, \ldots, \sigma_{n}\right)\right)-\sum_{i} f\left(\sigma_{1}, \ldots \llbracket \lambda X, \sigma_{i} \rrbracket, \sigma_{n}\right) .
$$

This flat structure coincides with the flat structure in $\Lambda^{n} \boldsymbol{g}^{*}$ defined in the paper $[\mathrm{K}-\mathrm{M}]$ via some system $\mathcal{A}=\left\{\tilde{\varphi}_{U}^{n}\right\}$ of local trivializations with locally-constant transitive functions. We recall that $\tilde{\varphi}_{U}^{n}: U \times \Lambda^{n} \mathfrak{g}^{*} \rightarrow \Lambda^{n} \boldsymbol{g}^{*}$ ( $\mathfrak{g}$ is the typical fiber of $\boldsymbol{g}$ ) is determined by a local trivialization $\varphi_{U}: A_{U} \rightarrow T U \times \mathfrak{g}$ of the Lie algebroid $A$ in the following way: $\varphi_{U}$ induces a local trivialization $\varphi_{U}^{+}: g_{U} \rightarrow U \times \mathfrak{g}$ of the adjoint Lie Algebra Bundle $\boldsymbol{g}$ and we put $\left(\tilde{\varphi}_{U}^{n}\right)_{x}=\Lambda^{n}\left(\varphi_{U}^{+}\right)_{x}^{*}$. 
Now, we carry over the differentials $d_{A, 1}^{j, i}: E_{A, 1}^{j, i} \rightarrow E_{A, 1}^{j+1, i}, d_{A_{c}, 1}^{j, i}: E_{A_{c}, 1}^{j, i} \rightarrow$ $E_{A_{c}, 1}^{j+1, i}$, to the spaces $\Omega^{j}\left(M, H_{\nabla^{+}}^{i}(\boldsymbol{g}, \xi)\right)$ and $\Omega_{c}^{j}\left(M, H_{\nabla^{+}}^{i}(\boldsymbol{g}, \xi)\right)$, respectively, via the isomorphisms $\Psi_{A, 1}$ and $\Psi_{A_{c}, 1}$. Since the canonical homomorphism

$$
\Gamma\left(H_{\nabla^{+}}^{i}(\boldsymbol{g}, \xi)\right) \rightarrow H_{\nabla \circ \iota}^{i}(\mathfrak{k}, \Gamma(\xi))
$$

is not a monomorphism unless the Lie algebra bundle $\boldsymbol{g}$ is trivial, we can not infer the form of this differentials immediately from the level of $\mathbb{R}$-cochains and its spectral sequence (5.2). In comparising of the cohomology classes from $H_{\nabla \circ \iota}^{i}(\mathfrak{k}, \Gamma(\xi))$ having representative of $C^{\infty}(M)$-linear cochains we must see whether these representatives differ by a $C^{\infty}(M)$-linear cochain.

Proposition 5.9. The following diagrams are commutative

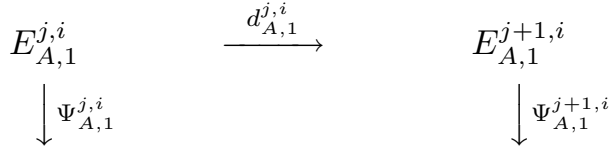

$$
\begin{aligned}
& \Omega^{j}\left(M, H_{\nabla^{+}}^{i}(\boldsymbol{g}, \xi)\right) \stackrel{(-1)^{i} d_{\nabla^{i}}}{\longrightarrow} \Omega^{j+1}\left(M, H_{\nabla^{+}}^{i}(\boldsymbol{g}, \xi)\right)
\end{aligned}
$$

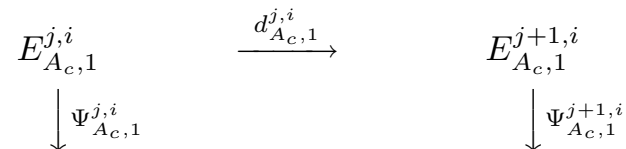

$$
\begin{aligned}
& \Omega_{c}^{j}\left(M, H_{\nabla^{+}}^{i}(\boldsymbol{g}, \xi)\right) \stackrel{(-1)^{i} d_{\nabla^{i}}}{\longrightarrow} \Omega_{c}^{j+1}\left(M, H_{\nabla^{+}}^{i}(\boldsymbol{g}, \xi)\right)
\end{aligned}
$$

Proof. The calculations identical as in the $\mathbb{R}$-linear cochains [H-S] yield for $f \in$ $Z_{A, 1}^{j, i} \subset Z_{1}^{j, i}$ the following formulae

$$
\begin{aligned}
& (-1)^{i}\left(d_{\nabla^{i}} \circ \Psi_{A, 1}^{j, i}[f]\right)\left(X_{1}, \ldots, X_{j+1}\right)=(-1)^{(j+1) i}\left[\rho_{f}\left(X_{1}, \ldots, X_{j+1}\right)\right], \\
& \left(\Psi_{A, 1}^{j+1, i} \circ d_{A, 1}^{j, i}[f]\right)\left(X_{1}, \ldots, X_{j+1}\right) \\
& =(-1)^{(j+1) i}\left[\rho_{f}\left(X_{1}, \ldots, X_{j+1}\right)-d_{\nabla^{+}}\left((-1)^{j} \bar{f}_{j+1}\left(X_{1}, \ldots, X_{j+1}\right)\right)\right]
\end{aligned}
$$

where $\rho_{f} \in \Omega^{j+1}\left(M, \Lambda^{i} \boldsymbol{g}^{*} \otimes \xi\right)$ and $\rho_{f}\left(X_{1}, \ldots, X_{j+1}\right)$ is a $d_{\nabla^{+}-\text {cocycle defined by }}$

$$
\begin{aligned}
& \rho_{f}\left(X_{1}, \ldots, X_{j+1}\right)\left(\sigma_{1}, \ldots, \sigma_{i}\right) \\
& =\sum_{t=1}^{j+1}(-1)^{t+1} \nabla_{\lambda X_{t}}\left(\bar{f}_{j}\left(X_{1}, \ldots \hat{t} \ldots, X_{j+1}\right)\left(\sigma_{1}, \ldots, \sigma_{i}\right)\right)+ \\
& +\sum_{t=1}^{j+1}(-1)^{t} \sum_{s=1}^{i} \bar{f}_{j}\left(X_{1}, \ldots \hat{t} \ldots, X_{j+1}\right)\left(\sigma_{1}, \ldots, \llbracket \lambda X_{t}, \sigma_{s} \rrbracket \sigma_{i}\right)+ \\
& +\sum_{r<s}(-1)^{r+s} \bar{f}_{j}\left(\left[X_{r}, X_{s}\right], X_{1}, \ldots \hat{r} \ldots \hat{s} \ldots X_{j+1}\right)\left(\sigma_{1}, \ldots, \sigma_{i}\right) .
\end{aligned}
$$


The cochain $\bar{f}_{j+1}\left(X_{1}, \ldots, X_{j+1}\right)$ is $C^{\infty}(M)$-linear, i.e. belongs to the module $\Omega^{j+1}\left(M, \Lambda^{i-1} \boldsymbol{g}^{*} \otimes \xi\right)$. This gives

$$
\begin{aligned}
& \left((-1)^{i} d_{\nabla^{i}} \circ \Psi_{A, 1}^{j, i}-\Psi_{A, 1}^{j+1, i} \circ d_{A, 1}^{j, i}\right)(f)\left(X_{1}, \ldots, X_{j+1}\right) \\
& =(-1)^{(j+1) i}\left[d_{\nabla^{+}}\left((-1)^{j} \bar{f}_{j+1}\left(X_{1}, \ldots, X_{j+1}\right)\right)\right] \\
& =0 .
\end{aligned}
$$

If $f$ has a compact support, the same hold for $\rho_{f}$ and $\bar{f}_{j+1}$ and we get the commutativity of the second diagram.

The next theorem is the main goal of this section. It describes the second terms of the sepectral sequences (5.4) and (5.6) (see also $[\mathrm{M}]$ ).

Theorem 5.10. The homomorphisms

$$
\begin{aligned}
\Psi_{A, 2}: E_{A, 2}^{j, i} \rightarrow H_{\nabla^{i}}^{j}\left(M, H_{\nabla^{+}}^{i}(\boldsymbol{g}, \xi)\right), & {[f] \mapsto\left[(-1)^{j i}\left[\bar{f}_{j}\right]\right] } \\
\Psi_{A_{c}, 2}: E_{A_{c}, 2}^{j, i} \rightarrow H_{\nabla^{i}, c}^{j}\left(M, H_{\nabla^{+}}^{i}(\boldsymbol{g}, \xi)\right), & {[f] \mapsto\left[(-1)^{j i}\left[\bar{f}_{j}\right]\right] }
\end{aligned}
$$

are isomorphisms of $C^{\infty}(M)$-modules.

Proof. Clearly, we need to notice only that $\Psi_{A, 2}$ is a composition of isomorphisms

$$
\Psi_{A, 2}: E_{A, 2}^{j, i} \stackrel{\sigma_{A, 1}^{j, i}}{\cong} H^{j}\left(E_{A, 1}^{*, i}, d_{A, 1}^{*, i}\right) \stackrel{\left(\Psi_{A, 1}^{j, i}\right)}{\cong} \# H_{\nabla^{i}}^{j}\left(M, H_{\nabla^{+}}^{i}(\boldsymbol{g}, \xi)\right)
$$

and analogously for compact supports.

\section{Algebroids AND PAIRINGS}

Assume that $A$ is a transitive Lie algebroid with three representations

$$
\nabla_{r}: A \rightarrow A\left(\xi_{r}\right), \quad r=1,2,3,
$$

and a pairing

$$
F: \xi_{1} \times \xi_{2} \rightarrow \xi_{3}
$$

compatible with the representations $\left(\nabla_{1}, \nabla_{2}, \nabla_{3}\right)$, i.e. fulfilling the property analogous to (2.5) in which we must replace $X$ by $\gamma \in \Gamma(A)$. Then the multiplication of cochains

$$
\wedge: \Lambda^{j} \boldsymbol{g}^{*} \otimes \xi_{1} \times \Lambda^{i} \boldsymbol{g}^{*} \otimes \xi_{2} \rightarrow \Lambda^{j+i} \boldsymbol{g}^{*} \otimes \xi_{3}
$$

is compatible with

(a) suitable representations $\mathcal{L}_{A}^{j}, \mathcal{L}_{A}^{i}, \mathcal{L}_{A}^{j+i}$

$$
\mathcal{L}_{A, \gamma}^{j+i}(f \wedge g)=\mathcal{L}_{A, \gamma}^{j+i}(f) \wedge g+f \wedge \mathcal{L}_{A, \gamma}^{j+i}(g),
$$

$f \in \Gamma\left(\Lambda^{j} \boldsymbol{g}^{*} \otimes \xi_{1}\right), g \in \Gamma\left(\Lambda^{i} \boldsymbol{g}^{*} \otimes \xi_{2}\right)$,

(b) differentials $d_{\nabla_{1}^{+}}, d_{\nabla_{2}^{+}}, d_{\nabla_{3}^{+}}$

$$
d_{\nabla_{3}^{+}}(f \wedge g)=d_{\nabla_{1}^{+}}(f) \wedge g+(-1)^{j} f \wedge d_{\nabla_{2}^{+}}(g)
$$

$f, g$ as above.

The latter equality gives the pairing of cohomology vector bundles

$$
\wedge: H_{\nabla_{1}^{+}}^{j}\left(\boldsymbol{g}, \xi_{1}\right) \times H_{\nabla_{2}^{+}}^{i}\left(\boldsymbol{g}, \xi_{2}\right) \rightarrow H_{\nabla_{3}^{+}}^{j+i}\left(\boldsymbol{g}, \xi_{3}\right)
$$


which is compatible with the suitable representations $\mathcal{L}_{A}^{\#, j}, \mathcal{L}_{A}^{\#, i}, \mathcal{L}_{A}^{\#, j+i}$ and finally with the flat covariant derivatives $\nabla^{j}, \nabla^{i}, \nabla^{j+i}$

$$
\nabla_{X}^{j+i}([f] \wedge[g])=\nabla_{X}^{j}([f]) \wedge[g]+[f] \wedge \nabla_{X}^{i}[g]
$$

We assume in the sequel that $n=\operatorname{rank} \boldsymbol{g}$ (and we recall that $m=\operatorname{dim} M$ ).

Together with three representations $\nabla_{r}$ one consider three graded filtered differential spaces $\Omega\left(A, \xi_{1}\right), \Omega_{c}\left(A, \xi_{2}\right), \Omega_{c}\left(A, \xi_{3}\right)(5.3)$, (5.5), and theirs spectral sequences $\left({ }^{1} E_{A, s}^{j, i},{ }^{1} d_{A, \nabla, s}\right),\left({ }^{2} E_{A_{c}, s}^{j, i},{ }^{2} d_{A_{c}, \nabla, s}\right),\left({ }^{3} E_{A_{c}, s}^{j, i},{ }^{3} d_{A_{c}, \nabla, s}\right)$. Using monomorphy of $\rho_{0}$ and of $\rho_{c, 0}$, Lemma 5.1, we see immediately from the case of $\mathbb{R}$-linear cochains [H-S] that the following diagram commutes.

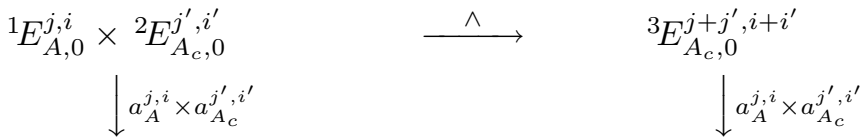

$$
\begin{aligned}
& \Omega^{j}\left(M, \Lambda^{i} \boldsymbol{g}^{*} \otimes \xi_{1}\right) \times \Omega_{c}^{j^{\prime}}\left(\Lambda^{i^{\prime}} \boldsymbol{g}^{*} \otimes \xi_{2}\right) \stackrel{(-1)^{i^{\prime} j} \cdot \wedge}{\longrightarrow} \Omega_{c}^{j+j^{\prime}}\left(\Lambda^{i+i^{\prime}} \boldsymbol{g}^{*} \otimes \xi_{3}\right) \text {. }
\end{aligned}
$$

Passing twice to cohomology and using definitions of suitable homomorphisms we get the commutativity of the diagram

$$
\begin{aligned}
& { }^{1} E_{A, 2}^{j, i} \times{ }^{2} E_{A_{c}, 2}^{j^{\prime}, i^{\prime}} \\
& \downarrow{ }^{1} \Psi_{A, 2}^{j, i} \times{ }^{2} \Psi_{A_{c}, 2}^{j^{\prime}, i^{\prime}} \\
& \stackrel{\wedge}{\longrightarrow} E_{A_{c}, 2}^{j+j^{\prime}, i+i^{\prime}} \\
& \downarrow{ }^{3} \Psi_{A_{c}, 2}^{j+j^{\prime}, i+i^{\prime}} \\
& H_{\nabla^{i}}^{j}\left(M, H_{\nabla_{1}^{+}}^{i}\left(\boldsymbol{g}, \xi_{1}\right)\right) \times H_{\nabla^{i^{\prime}, c}}^{j^{\prime}}\left(M, H_{\nabla_{2}^{+}}^{i^{\prime}}\left(\boldsymbol{g}, \xi_{2}\right)\right) \stackrel{(-1)^{i^{\prime} j} \cdot \wedge}{\longrightarrow} H_{\nabla^{i+i^{\prime}, c}}^{j+j^{\prime}}\left(M, H_{\nabla_{3}^{+}}^{i+i^{\prime}}\left(\boldsymbol{g}, \xi_{3}\right)\right) .
\end{aligned}
$$

The main theorem of Chapter 1 one gets the very important

Conclusion 6.1. If $\xi_{3}$ is a line bundle and $\left(H_{\nabla_{3}^{+}}^{n}\left(\boldsymbol{g}, \xi_{3}\right), \nabla^{n}\right) \sim\left(\right.$ or $\left.(M), \partial^{\text {or }}\right)$ [in particular, $\left(\nabla_{3}^{+}\right)_{x}=\nabla_{\text {trad }} \otimes \mathrm{id}$ according to Lemma 3.5] and the pairing of cohomology vector bundles

$$
\wedge: H_{\nabla_{1}^{+}}^{i}\left(\boldsymbol{g}, \xi_{1}\right) \times H_{\nabla_{2}^{+}}^{n-i}\left(\boldsymbol{g}, \xi_{2}\right) \rightarrow H_{\nabla_{3}^{+}}^{n}\left(\boldsymbol{g}, \xi_{3}\right)
$$

is nondegenerate, then the same holds for the pairing

$H_{\nabla^{i}}^{j}\left(M, H_{\nabla_{1}^{+}}^{i}\left(\boldsymbol{g}, \xi_{1}\right)\right) \times H_{\nabla^{n-i}, c}^{m-j}\left(M, H_{\nabla_{2}^{+}}^{n-i}\left(\boldsymbol{g}, \xi_{2}\right)\right) \rightarrow H_{\nabla^{n}, c}^{m}\left(M, H_{\nabla_{3}^{+}}^{n}\left(\boldsymbol{g}, \xi_{3}\right)\right) \stackrel{\int_{M}^{\#}}{\longrightarrow} \mathbb{R}$.

i.e.

$$
H_{\nabla^{i}}^{j}\left(M, H_{\nabla_{1}^{+}}^{i}\left(\boldsymbol{g}, \xi_{1}\right)\right) \cong\left(H_{\nabla^{n-i}, c}^{m-j}\left(M, H_{\nabla_{2}^{+}}^{n-i}\left(\boldsymbol{g}, \xi_{2}\right)\right)\right)^{*} .
$$

Diagram (6.2) assert that the nondegenerate pairing (6.3) is \pm equal to the multiplication of the second term of the spectral sequences

$$
{ }^{1} E_{A, 2}^{j, i} \times{ }^{2} E_{A_{c}, 2}^{m-j, n-i} \stackrel{\wedge}{\longrightarrow} E_{A_{c}, 2}^{m, n} \stackrel{\cong}{\longrightarrow}
$$

so the last is nondegenerate as well,

$$
{ }^{1} E_{A, 2}^{j, i} \cong\left({ }^{2} E_{A_{c}, 2}^{m-j, n-i}\right)^{*},
$$

and the main theorem of Chapter 4 gives that the multiplication of cohomology classes

$$
\langle\cdot, \cdot\rangle_{H}: H_{\nabla_{1}}^{j}\left(A, \xi_{1}\right) \times H_{\nabla_{2}, c}^{m+n-j}\left(A, \xi_{2}\right) \rightarrow H_{\nabla_{3}, c}^{m+n}\left(A, \xi_{3}\right) \stackrel{\cong}{\longrightarrow}
$$


is nondegenerate too, i.e.

$$
H_{\nabla_{1}}^{j}\left(A, \xi_{1}\right) \cong\left(H_{\nabla_{2}, c}^{m+n-j}\left(A, \xi_{2}\right)\right)^{*} .
$$

\section{Evens-Lu-Weinstein PAIRING FOR transitive Lie ALgeBroids}

7.1. Nondegeneracy of Evens-Lu-Weinstein pairing for transitive Lie algebroids. We prove that for transitive Lie algebroid $A$ the duality of Evens-LuWeinstein $[\mathrm{E}-\mathrm{L}-\mathrm{W}]$

$$
H^{j}(A) \times H_{D^{o r}, c}^{m+n-j}\left(A, Q_{A}^{o r}\right) \rightarrow H_{D^{\text {or }}, c}^{m+n}\left(A, Q_{A}^{o r}\right) \rightarrow \mathbb{R}
$$

is nondegenerate, i.e. $H_{D^{o r}, c}^{m+n}\left(A, Q_{A}^{o r}\right) \cong \mathbb{R}$ and

$$
H^{j}(A) \cong\left(H_{D^{o r}, c}^{m+n-j}\left(A, Q_{A}^{o r}\right)\right)^{*} .
$$

For arbitrary (nonregular in general) Lie algebroid $A$ on a manifold $M$ the authors $[\mathrm{E}-\mathrm{L}-\mathrm{W}]$ introduced a vector bundle

$$
Q_{A}=\Lambda^{t o p} A \otimes \Lambda^{t o p} T^{*} M
$$

(the notation $\Lambda^{t o p}$ refers to the highest exterior power). Geometrically, sections of $Q_{A}$ can be thought of as transverse measures to characteristic foliation $\operatorname{Im} \#_{A}$ to any Lie algebroid $A[\mathrm{E}-\mathrm{L}-\mathrm{W}]$. For Poisson manifolds, the Evens-Lu-Weinstein pairings takes the form of the pairing on the Poisson homology; for more applications see [E-L-W]. Ibidem, there is an example of nonregular Lie algebroid $A$ over a compact oriented manifold for which the pairing $H^{j}(A) \times H_{D, c}^{m+n-j}\left(A, Q_{A}\right) \rightarrow \mathbb{R}$ is not necessarily nondegenerate. J.Huebschmann in $[\mathrm{H}]$ has generalized the construction of the bundle $Q_{A}$ and the modular class $\theta_{A}$ to Lie-Rinehart algebras, an algebraic generalization of Lie algebroids.

We slightly modify the Weinstein construction to consider nonoriented manifolds:

$$
Q_{A}^{o r}=Q_{A} \otimes \text { or }(M) .
$$

For an oriented manifold $M$ we can identify $Q_{A}^{o r}=Q_{A}$.

In $[\mathrm{E}-\mathrm{L}-\mathrm{W}]$ a representation

$$
D: A \rightarrow A\left(Q_{A}\right)
$$

was introduced by

$$
D_{\gamma}(Y \otimes \varphi)=L_{\gamma}(Y) \otimes \varphi+Y \otimes L_{\#_{A}(\gamma)}(\varphi),
$$

$Y \in \Gamma\left(\Lambda^{t o p} A\right), \varphi \in \Gamma\left(\Lambda^{t o p} T^{*} M\right)=\Omega^{m}(M)$, where $L_{\gamma}(Y)=[\gamma, Y]([\gamma, Y]$ denotes the Schouten bracket) and $L_{\#_{A}(\gamma)}(\varphi)$ is the usual Lie derivative of a differential form $\varphi$. We recall that for $Y=\gamma_{1} \wedge \ldots \wedge \gamma_{t}$

$$
L_{\gamma}(Y)=\sum_{i} \gamma_{1} \wedge \ldots \wedge \llbracket \gamma, \gamma_{i} \rrbracket \wedge \ldots \wedge \gamma_{t} .
$$

There is some interest to consider the representation $D$ in the context of intrinsic characteristic classes of Lie algebroids $[\mathrm{C}],[\mathrm{F}]$.

We modify the representation $D$ to

$$
D^{o r}=D \otimes \partial_{A}^{o r}: A \rightarrow A\left(Q_{A}^{o r}\right) .
$$


In the sequel we will be interested only in the transitive case. A choice of a connection $\lambda: T M \rightarrow A$ enables us to identify

$$
\begin{aligned}
\Lambda^{m+n} A & =\Lambda^{n} \boldsymbol{g} \otimes \Lambda^{m} T M, \\
\varepsilon \wedge\left(\Lambda^{m} \lambda\right)(X) & =\varepsilon \otimes Y,
\end{aligned}
$$

and

$$
Q_{A}^{o r}=\Lambda^{n} \boldsymbol{g} \otimes \Lambda^{m} T M \otimes \Lambda^{m} T^{*} M \otimes \text { or }(M)=\Lambda^{n} \boldsymbol{g} \otimes \text { or }(M) .
$$

Lemma 7.1. (a) $D^{+}: \boldsymbol{g} \rightarrow$ End $\left(Q_{A}\right)$ is defined by

$$
D_{\sigma}^{+}=\left(\nabla_{\mathrm{trad}}\right)_{\sigma}=\operatorname{tr}\left(\operatorname{ad}_{\sigma}\right) \cdot \mathrm{id}, \quad \sigma \in \Gamma(\boldsymbol{g}) .
$$

(b) $H_{D^{+}}^{n}\left(\boldsymbol{g}, Q_{A}\right)=\Lambda^{n} \boldsymbol{g}^{*} \otimes Q_{A}, H_{D^{o r+}}^{n}\left(\boldsymbol{g}, Q_{A}^{o r}\right)=\Lambda^{n} \boldsymbol{g}^{*} \otimes Q_{A}^{o r}$.

Proof. (a) Consider locally defined nonsingular section of $Q_{A}$ of the form $\varepsilon_{U} \otimes$ $X_{U} \otimes \varphi_{U}, \varepsilon_{U} \in \Gamma\left(\Lambda^{n} \boldsymbol{g}_{U}\right), X_{U} \in \Gamma\left(\Lambda^{m} T M_{U}\right), \varphi_{U} \in \Gamma\left(\Lambda^{m} T^{*} M_{U}\right)$, and assume that $\left\langle X_{U}, \varphi_{U}\right\rangle=1$. For $\sigma \in \Gamma(\boldsymbol{g})$ we have $\#_{A}(\sigma)=0$ and $\llbracket \sigma, \lambda W_{i} \rrbracket \in \Gamma(\boldsymbol{g})$. Therefore if $\varepsilon_{U}=\sigma_{1} \wedge \ldots \wedge \sigma_{n}, X_{U}=W_{1} \wedge \ldots \wedge W_{m}, \sigma_{i} \in \Gamma(\boldsymbol{g}), W_{i} \in \Gamma\left(T M_{U}\right)$,

$$
\begin{aligned}
& D_{\sigma}\left(\varepsilon_{U} \otimes X_{U} \otimes \varphi_{U}\right) \\
& =L_{\sigma}\left(\varepsilon_{U} \otimes X_{U}\right) \otimes \varphi_{U} \\
& =L_{\sigma}\left(\sigma_{1} \wedge \ldots \wedge \sigma_{n} \wedge \lambda W_{1} \wedge \ldots \wedge \lambda W_{m}\right) \otimes \varphi_{U} \\
& =\sum_{i} \sigma_{1} \wedge \ldots \wedge \llbracket \sigma, \sigma_{i} \rrbracket \wedge \ldots \wedge \sigma_{n} \wedge \lambda W_{1} \wedge \ldots \wedge \lambda W_{m} \otimes \varphi_{U} \\
& =\operatorname{tr}\left(\operatorname{ad}_{\sigma}\right) \cdot \varepsilon_{U} \otimes X_{U} \otimes \varphi_{U} .
\end{aligned}
$$

(b) Follows immediately from Proposition 3.2.

The vector bundle $\Lambda^{n} \boldsymbol{g}^{*} \otimes Q_{A}$ is trivial. Indeed, the classical homomorphism

$$
\begin{gathered}
c: \Lambda^{n} \boldsymbol{g}^{*} \otimes Q_{A}=\Lambda^{n} \boldsymbol{g}^{*} \otimes \Lambda^{n} \boldsymbol{g} \otimes \Lambda^{m} T M \otimes \Lambda^{m} T^{*} M \rightarrow M \times \mathbb{R} \\
c\left(\varepsilon^{*} \otimes \varepsilon \otimes X \otimes \varphi\right)=\left\langle\varepsilon^{*}, \varepsilon\right\rangle \cdot\langle X, \varphi\rangle
\end{gathered}
$$

is an isomorphism. Therefore

$$
c \otimes i d: \Lambda^{n} \boldsymbol{g}^{*} \otimes Q_{A}^{\text {or }} \stackrel{\cong}{\longrightarrow} \text { or }(M) .
$$

Let $A(c): A\left(\Lambda^{n} \boldsymbol{g}^{*} \otimes Q_{A}\right) \rightarrow A(M \times \mathbb{R})$ be the induced isomorphism of Lie algebroids $[\mathrm{K} 1]$,

$$
A(c)(u)(f)=c\left(u\left(c^{-1} \circ f\right)\right)
$$

$u \in \Gamma\left(A\left(\Lambda^{n} \boldsymbol{g}^{*} \otimes Q_{A}\right)\right), f \in C^{\infty}(M)$. Let

$$
\nabla^{D}: T M \rightarrow A\left(H_{D^{+}}^{n}\left(\boldsymbol{g}, Q_{A}\right)\right)=A\left(\Lambda^{n} \boldsymbol{g}^{*} \otimes Q_{A}\right)
$$

be the induced flat adjoint covariant derivative (5.9) for $D$. Analogously we have $\nabla^{D^{o r}}: T M \rightarrow A\left(\Lambda^{n} \boldsymbol{g}^{*} \otimes Q_{A}^{o r}\right)$.

Lemma 7.2. The compositions

$$
\begin{array}{r}
\nabla^{D}: T M \rightarrow A\left(\Lambda^{n} \boldsymbol{g}^{*} \otimes Q_{A}\right) \stackrel{A(c)}{\cong} A(T M \times \mathbb{R}), \\
\nabla^{D^{o r}}: T M \rightarrow A\left(\Lambda^{n} \boldsymbol{g}^{*} \otimes Q_{A}^{o r}\right) \stackrel{A(c \otimes i d)}{\stackrel{(⿻)}{\cong}} A(\operatorname{or}(M)),
\end{array}
$$


are trivial representations $\partial$ and $\partial^{\text {or }}$, respectively, so

$$
\begin{aligned}
\left(H_{D^{+}}^{n}\left(\boldsymbol{g}, Q_{A}\right), \nabla^{D}\right) & \sim(T M \times \mathbb{R}, \partial), \\
\left(H_{D^{\text {or }}}^{n}\left(\boldsymbol{g}, Q_{A}^{o r}\right), \nabla^{D^{o r}}\right) & \sim\left(\text { or }(M), \partial^{o r}\right) .
\end{aligned}
$$

Proof. It is necessary to check it locally. Take locally defined nonsingular sections $\varepsilon_{U} \in \Gamma\left(\Lambda^{n} \boldsymbol{g}_{U}\right), X_{U} \in \Gamma\left(\Lambda^{m} T M_{U}\right)$ and theirs duals $\varepsilon_{U}^{*} \in \Gamma\left(\Lambda^{n} \boldsymbol{g}_{U}^{*}\right), \varphi_{U} \in$ $\Gamma\left(\Lambda^{m} T^{*} M_{U}\right),\left\langle\varepsilon_{U}^{*}, \varepsilon_{U}\right\rangle=1,\left\langle X_{U}, \varphi_{U}\right\rangle=1$. On the set $U$ arbitrary section of the bundle $\Lambda^{n} \boldsymbol{g}^{*} \otimes Q_{A}=\Lambda^{n} \boldsymbol{g}^{*} \otimes \Lambda^{n} \boldsymbol{g} \otimes \Lambda^{m} T M \otimes \Lambda^{m} T^{*} M$ is of the form $f \cdot \varepsilon_{U}^{*} \otimes$ $\varepsilon_{U} \otimes X_{U} \otimes \varphi_{U}, f \in C^{\infty}(U)$. For $X \in \mathfrak{X}\left(M_{U}\right), X_{U}=W_{1} \wedge \ldots \wedge W_{m}\left(W_{1}, \ldots, W_{m}\right.$ is a base of vector fields on $U$ ) and $\varphi_{U}=W_{1}^{*} \wedge \ldots \wedge W_{m}^{*}, W_{i}^{*}$ is the dual basis, and $\varepsilon_{U}=\sigma_{1} \wedge \ldots \wedge \sigma_{n}$ ( $\sigma_{i}$ is a base of the vector bundle $\boldsymbol{g}$ on $\left.U\right)$, we write $\llbracket \lambda X, \sigma_{i} \rrbracket=\sum_{j} g_{i}^{j} \cdot \sigma_{j}, \llbracket \lambda X, \lambda W_{i} \rrbracket=\sum_{k} h_{i}^{k} \cdot \sigma_{k}+a_{i}^{k} \cdot \lambda_{k}$, so $\left[X, W_{i}\right]=a_{i}^{k} \cdot W_{k}$. Then

$$
\begin{aligned}
& D_{\lambda X}\left(\varepsilon_{U} \otimes X_{U} \otimes \varphi_{U}\right) \\
& =L_{\lambda X}\left(\varepsilon_{U} \otimes X_{U}\right) \otimes \varphi_{U}+\varepsilon_{U} \otimes X_{U} \otimes L_{X} \varphi_{U} \\
& =\left(\sum_{i} g_{i}^{i} \cdot \sigma \wedge \lambda X\right) \otimes \varphi_{U}+\sigma \wedge\left(\sum_{i} a_{i}^{i} \cdot \lambda X\right) \otimes \varphi_{U}+\sigma \wedge \lambda X \otimes \sum\left(-a_{i}^{i}\right) \varphi_{U} \\
& =\sum_{i} g_{i}^{i} \cdot \varepsilon_{U} \otimes X_{U} \otimes \varphi_{U} .
\end{aligned}
$$

Therefore

$$
\begin{aligned}
& \nabla_{X}^{D}\left(f \cdot \varepsilon_{U}^{*} \otimes \varepsilon_{U} \otimes X_{U} \otimes \varphi_{U}\right)\left(\varepsilon_{U}\right) \\
& =D_{\lambda X}\left(f \cdot \varepsilon_{U} \otimes X_{U} \otimes \varphi_{U}\right)- \\
& -\sum_{i} f \cdot \varepsilon_{U}^{*}\left(\sigma_{1} \wedge \ldots \wedge \llbracket \lambda X, \sigma_{i} \rrbracket \wedge \ldots \wedge \sigma_{n}\right) \cdot \varepsilon_{U} \otimes X_{U} \otimes \varphi_{U} \\
& =\partial_{X} f \cdot \varepsilon_{U} \otimes X_{U} \otimes \varphi_{U}+f \cdot D_{\lambda X}\left(\varepsilon_{U} \otimes X_{U} \otimes \varphi_{U}\right)-f \cdot \sum_{i} g_{i}^{i} \cdot \varepsilon_{U} \otimes X_{U} \otimes \varphi_{U} \\
& =\partial_{X} f \cdot \varepsilon_{U} \otimes X_{U} \otimes \varphi_{U} \\
& =\partial_{X} f \cdot\left(\varepsilon_{U}^{*} \otimes \varepsilon_{U} \otimes X_{U} \otimes \varphi_{U}\right)\left(\varepsilon_{U}\right) .
\end{aligned}
$$

Finally

$$
\begin{aligned}
\left(A(c) \circ \nabla_{X}^{D}\right)(f) & =c\left(\nabla_{X}^{D}\left(f \cdot \varepsilon_{U}^{*} \otimes \varepsilon_{U} \otimes X_{U} \otimes \varphi_{U}\right)\right) \\
& =c\left(\partial_{X} f \cdot\left(\varepsilon_{U}^{*} \otimes \varepsilon_{U} \otimes X_{U} \otimes \varphi_{U}\right)\right) \\
& =\partial_{X} f .
\end{aligned}
$$

For the proof of the second part we notice that for local $\partial^{\text {or }}$-constant section $\sigma_{0}$ of or $\left(M_{U}\right)$ one has

$$
\begin{aligned}
\left(A(c \otimes i d) \circ \nabla_{X}^{D^{o r}}\right)\left(f \otimes \sigma_{0}\right) & =\left(A(c) \circ \nabla_{X}^{D}\right)(f) \otimes \sigma_{0} \\
& =\partial_{X} f \otimes \sigma_{0} \\
& =\partial_{X}^{o r}\left(f \otimes \sigma_{0}\right) .
\end{aligned}
$$

Theorem 7.3. For an arbitrary transitive Lie algebroid $A$

$$
H_{D^{o r}, c}^{m+n}\left(A, Q_{A}^{o r}\right) \cong \mathbb{R}
$$


and the Evens-Lu-Weinstein cohomology pairing

$$
H^{j}(A) \times H_{D^{o r}, c}^{m+n-j}\left(A, Q_{A}^{o r}\right) \rightarrow H_{D^{o r}, c}^{m+n}\left(A, Q_{A}^{o r}\right) \cong \mathbb{R}
$$

is nondegenerate, i.e.

$$
H^{j}(A) \cong\left(H_{D^{o r}, c}^{m+n-j}\left(A, Q_{A}^{o r}\right)\right)^{*} .
$$

Proof. Conclusion 3.4 and Lemma 7.1 show that the pairing

$$
H^{i}(\boldsymbol{g}) \times H_{D^{\text {or }}}^{n-i}\left(\boldsymbol{g}, Q_{A}^{\text {or }}\right) \rightarrow H_{D^{\text {or }}}^{n}\left(\boldsymbol{g}, Q_{A}^{\text {or }}\right)
$$

is nondegenerate. On account of Theorem 2.3 and Conclusion 6.1 we assert that the pairing

$H_{\nabla^{D}}^{j}\left(M, H^{i}(\boldsymbol{g})\right) \times H_{\nabla^{o r}, c}^{m-j}\left(M, H_{D^{o r+}}^{n-i}\left(\boldsymbol{g}, Q_{A}^{o r}\right)\right) \rightarrow H_{\nabla^{D^{o r}}, c}^{m}\left(M, H_{D^{o r+}}^{n}\left(\boldsymbol{g}, Q_{A}^{o r}\right)\right) \cong \mathbb{R}$,

is nondegenerate. Equivalently, this is a multiplication of the second terms of the Hochshild-Serre spectral sequences of graded filtered differential spaces $\Omega(A)$ with the trivial differential and $\Omega_{c}\left(A, Q_{A}^{o r}\right)$ with the differential $D^{o r}$. The fundamental Theorem 4.4, see also mentioned above Conclusion 6.1, completes the proof.

7.2. Remarks on the top group of cohomology. Analyzing the proof of Theorem 4.4 and composing isomorphism (7.3) with isomorphism (2.4) we can define the isomorphism $I: H_{D^{o r}, c}^{m+n}\left(A, Q_{A}^{o r}\right) \stackrel{\cong}{\longrightarrow} \mathbb{R}$ as a composition

$$
\begin{aligned}
I & : H_{D^{o r}, c}^{m+n}\left(A, Q_{A}^{o r}\right)=H_{D^{o r}, c}^{m, n}\left(A, Q_{A}^{o r}\right)=E_{0}^{m, n}\left(H_{D^{o r}, c}\left(A, Q_{A}^{o r}\right)\right) \stackrel{\sigma_{A_{c}, \infty}^{\sigma_{c}, n}}{\cong} E_{A_{c}, \infty}^{m, n}= \\
& =E_{A_{c}, 2}^{m, n} \stackrel{\Psi_{A_{c}, 2}^{m, n}}{\cong} H_{\nabla^{D^{o r},}, c}^{m}\left(M, H_{D^{o r+}}^{n}\left(\boldsymbol{g}, Q_{A}^{o r}\right)\right)= \\
& =H_{\nabla^{D^{o r}, c}}^{m}\left(M, \Lambda^{n} \boldsymbol{g}^{*} \otimes Q_{A}^{o r}\right) \stackrel{(c \otimes i d)_{\#}}{\cong} H_{\partial^{o r}, c}^{m}(M, \text { or }(M)) \stackrel{\int_{M}^{o r, \#}}{\cong} \mathbb{R} .
\end{aligned}
$$

We compare this isomorphism with the one defined by direct formula in [E$\mathrm{L}-\mathrm{W}]$ resctricting our interest to transitive Lie algebroids. Immediately from the definition of $\Psi_{A_{c}, 2}^{m, n}$ (see Theorem 5.10), $\Psi_{A_{c}, 2}^{m, n}[f]=(-1)^{m n}\left[\bar{f}_{m}\right]$, and definition of $\sigma_{A_{c}, \infty}^{m, n}$ we observe that

$$
I_{1}: H_{D^{o r}, c}^{m+n}\left(A, Q_{A}^{o r}\right)=E_{0}^{m, n} \underset{\cong}{\stackrel{\sigma_{A_{c}, \infty}^{m, n}}{\cong}} E_{A_{c}, \infty}^{m, n}=E_{A_{c}, 2}^{m, n} \stackrel{\Psi_{A_{c}, 2}^{m, n}}{\cong} H_{\nabla^{D^{o r},}, c}^{m}\left(M, \Lambda^{n} \boldsymbol{g}^{*} \otimes Q_{A}^{o r}\right)
$$

is given by the formula looking analogously to $\Psi_{A_{c}, 2}^{m, n}, I_{1}[f]=(-1)^{m n}\left[\bar{f}_{m}\right]$, or equivalently (under the identification $\Lambda^{m+n} A^{*}=\Lambda^{m} T^{*} M \otimes \Lambda^{n} \boldsymbol{g}^{*}$ given by the help of a connection $\lambda: T M \rightarrow A)$ by

$$
I_{1}\left(\left(\varphi \otimes \varepsilon^{*}\right) \otimes q\right)=(-1)^{m n}\left[\varphi \otimes\left(\varepsilon^{*} \otimes q\right)\right]
$$

where $\varphi \in \Omega_{c}^{m}(M), \varepsilon^{*} \in \Gamma\left(\Lambda^{n} \boldsymbol{g}^{*}\right), q \in \Gamma\left(Q_{A}^{o r}\right)$. Therefore if $q=\varepsilon \otimes X \otimes \mu \otimes e$, $\varepsilon \in \Gamma\left(\Lambda^{n} \boldsymbol{g}\right), X \in \Gamma\left(\Lambda^{m} T M\right), \mu \in \Gamma\left(\Lambda^{m} T^{*} M\right)=\Omega^{m}(M), e \in \Gamma($ or $(M))$ then

$$
(c \otimes i d)_{\#} \circ I_{1}\left(\left(\varphi \otimes \varepsilon^{*}\right) \otimes \varepsilon \otimes X \otimes \mu \otimes e\right)=(-1)^{m n}\left[\varphi \cdot\left\langle\varepsilon^{*}, \varepsilon\right\rangle \cdot\langle X, \mu\rangle \otimes e\right] .
$$

So, for $f=\left(\varphi \otimes \varepsilon^{*}\right) \otimes \varepsilon \otimes X \otimes \mu \otimes e$ we get

$$
\begin{aligned}
I[f] & =(-1)^{m n} \int_{M}^{o r}\left\langle\varepsilon^{*}, \varepsilon\right\rangle \cdot\langle X, \mu\rangle \cdot \varphi \otimes e=(-1)^{m n} \int_{M}^{o r}\left\langle\varepsilon^{*}, \varepsilon\right\rangle \cdot\langle X, \varphi\rangle \cdot \mu \otimes e \\
& =(-1)^{m n} \int_{M}^{o r}\left\langle\varphi \otimes \varepsilon^{*}, X \otimes \varepsilon\right\rangle \cdot \mu \otimes e
\end{aligned}
$$


which is concordant up to the sign with the definition of Evens-Lu-Weinstein [E-L-W] given by them only for oriented compact manifold (but for any Lie algebroid, not necessary transitive).

The fact $H_{D^{o r}, c}^{m+n}\left(A, Q_{A}^{o r}\right) \stackrel{\cong}{\longrightarrow}$ for transitive Lie algebroids is not proved in [E-L-W]. Below we prove this immediately without use of the spectral sequences.

(a) on oriented manifolds. The authors of [E-L-W] introduced an isomorphism of vector bundles

$$
\begin{gathered}
\tilde{\rho}: \Lambda^{\mathrm{top}} A^{*} \otimes \Lambda^{\mathrm{top}} A \otimes \Lambda^{\mathrm{top}} T^{*} M \rightarrow \Lambda^{\mathrm{top}} T^{*} M, \\
\tilde{\rho}(\Psi \otimes Y \otimes \mu)=\langle\Psi, Y\rangle \cdot \mu
\end{gathered}
$$

and proved a version of Stokes Theorem (to be sure for compact manifold but without troubles we can extend it to differential forms with compact support on arbitrary oriented manifold).

Theorem 7.4 (Stokes Theorem [E-L-W]). Let $\operatorname{rank} A=r$. For $r-1$-form $\Psi^{\prime} \in$ $\Gamma\left(\Lambda^{r-1} A^{*}\right)$ we have

$$
\tilde{\rho}\left(d_{D}\left(\Psi^{\prime} \otimes Y \otimes \mu\right)\right)=(-1)^{r-1} d_{d R}\left(\iota_{\left.\#_{A}\left(\Psi^{\prime}\right\lrcorner Y\right)} \mu\right) .
$$

Consequently, if the form $\Psi^{\prime} \otimes Y \otimes \mu$ has compact support then

$$
\int_{M} \tilde{\rho}\left(d_{D}\left(\Psi^{\prime} \otimes Y \otimes \mu\right)\right)=0 .
$$

Put

$$
\left.\left.\tilde{\rho}^{r-1}: \Lambda^{r-1} A^{*} \otimes Q_{A} \rightarrow \Lambda^{m-1} T^{*} M, \quad\left(\Psi^{\prime} \otimes Y \otimes \mu\right) \mapsto(-1)^{r-1} \iota_{\#_{A}\left(\Psi^{\prime}\right.}\right\lrcorner Y\right) \mu,
$$

and notice the commutativity of the diagram

$$
\begin{array}{ccc}
\Omega_{c}^{r-1}\left(A, Q_{A}\right) & \stackrel{\tilde{\rho}_{c}^{r-1}}{\longrightarrow} & \Omega_{c}^{m-1}(M) \\
\downarrow d_{D} & & \\
\Omega_{c}^{r}\left(A, Q_{A}\right) & \stackrel{\tilde{\rho}_{c}}{\longrightarrow} & \Omega_{c}^{m}(M) .
\end{array}
$$

From this we deduce that $\tilde{\rho}_{c}$ induces an $\mathbb{R}$-linear homomorphism in cohomology

$$
\tilde{\rho}_{c, \#}: H_{D, c}^{r}\left(A, Q_{A}\right) \rightarrow H_{c}^{r}(M) .
$$

Since $\tilde{\rho}$ is an isomorphism $\tilde{\rho}_{c, \#}$ is an epimorphism.

Lemma 7.5. If $A$ is transitive Lie algebroid, then $\tilde{\rho}_{c, \#}$ is an isomorphism.

Proof. One can easily see the lemma provided that $\tilde{\rho}^{r-1}$ is an epimorphism. It is a simple matter to show that $\tilde{\rho}_{x}^{r-1}$ is an epimorphism at every point $x \in M$ using transitivity of the Lie algebroid $A$ i.e. using the fact that the anchor $\left(\#_{A}\right)_{x}: A_{x} \rightarrow$ $T_{x} M$ is an epimorphism. This finishes the proof that

$$
\tilde{\rho}_{c, \#}: H_{D, c}^{r}\left(A, Q_{A}\right) \rightarrow H_{c}^{r}(M) \underset{\cong}{\stackrel{\int_{M}^{\#}}{\cong}} \mathbb{R}
$$

is an isomorphism.

(b) on nonoriented manifolds. We prove this analogously multiplying the vector bundles by or $(M)$ and use the Stokes theorem for densities [B-T]. 
7.3. Exceptional property of the Evens-Lu-Weinstein representation. Assume $A$ is a transitive Lie algebroid. Before the next theorems we must give algebroid's equivalent of some lemmas from Chapter 1 . For any $A$-connection $\nabla: A \rightarrow A(\xi)$ and a 1 -form $\omega \in \Omega^{1}(A)$ we define a new $A$-connection

$$
\nabla^{\omega}: A \rightarrow A(\xi), \quad \nabla_{\gamma}^{\omega} \nu=\nabla_{\gamma} \nu+\omega(\gamma) \cdot \nu
$$

The curvature tensors $R^{\nabla^{\omega}}, R^{\nabla} \in \Omega^{2}(A, \xi)$ of the connections $\nabla^{\omega}$ and $\nabla$ are related via the formula

$$
R^{\nabla^{\omega}}=R^{\nabla}+d_{A} \omega \otimes i d .
$$

Therefore, if $\nabla$ is flat (it means, $\nabla$ is a representation) then $\nabla^{\omega}$ is flat if and only if $\omega$ is closed. Each $A$-connection $\nabla: A \rightarrow A(M \times \mathbb{R})$ in the trivial vector bundle $M \times \mathbb{R}$ is of the form $\partial_{A}^{\omega}$, indeed, we need to put $\omega(\gamma)=\nabla_{\gamma}(1)$.

For a line bundle $\xi$ and a representation $\nabla: A \rightarrow A(\xi)$ the differential equation $\nabla \nu=0$ is locally uniquelly integrable provided that it is locally integrable.

Lemma 7.6. For a line bundle $\xi$ and a representation $\nabla: A \rightarrow A(\xi)$ the differential equation $\nabla \nu=0$ is locally integrable if and only if $\nabla^{+}=0$. This last condition is equivalent to the projectability of $\nabla$, i.e. that $\nabla=\tilde{\nabla} \circ \#_{A}$ for some usual flat covariant derivative $\tilde{\nabla}$ on $M$ in the vector bundle $\xi$.

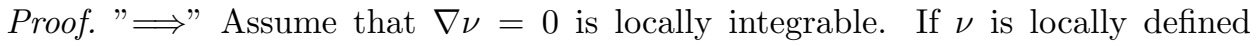
nonsingular $\nabla$-constant section of $\xi$ then arbitrary section is equal to $\nu_{1}=f \cdot \nu$ for a smooth function $f$ and for $\sigma \in \Gamma(\boldsymbol{g})$

$$
\nabla_{\sigma}^{+}(f \cdot \nu)=\partial_{\#_{A}(\sigma)} f \cdot \nu+f \cdot \nabla_{\sigma} \nu=0 .
$$

$" \Longleftarrow "$ Assume that $\nabla^{+}=0$. Take $x_{0} \in M$ and $u \in \xi_{x_{0}}$. Locally $\left(x_{0} \in U \cong \mathbb{R}^{m}\right)$ $\nabla_{U}=\partial_{A_{U}}^{\omega}: A_{U} \rightarrow A\left(\xi_{U}\right)=A(U \times \mathbb{R})$ for a closed 1-form $\omega \in \Omega^{1}\left(A_{U}\right)$. By assumption $\nabla^{+}=0, \omega(\sigma) \cdot \nu=0$ for all $\sigma \in \Gamma(\boldsymbol{g})$ and $\nu \in \Gamma(\xi)$, so $\omega(\sigma)=0$ and $\omega$ is projectable on $U, \omega=\#_{A}^{*}(\bar{\omega})$ for some $\bar{\omega} \in \Omega^{1}(U)$. Since the anchor $\#_{A}$ is an epimorphism, the pullback of the differential forms $\#_{A}^{*}$ is a monomorphism. Therefore, since $0=d_{A} \omega=d_{A}\left(\#_{A}^{*}(\bar{\omega})\right)=\#_{A}^{*}\left(d_{d R} \bar{\omega}\right)$ we get $d_{d R} \bar{\omega}=0$. Clearly, then $\bar{\omega}=d f$ for some function $f \in C^{\infty}(U)$. It is easy to see that the section $\sigma=e^{-f}$ of the bundle $U \times \mathbb{R} \cong \xi_{U}$ is $\nabla_{U^{-}}$constant.

Similar considerations show that for trivial vector bundle $\xi=M \times \mathbb{R}$ and a representation $\partial_{A}^{\omega}$ the following conditions are equivalent (1) $\nabla^{+}=0,(2) \omega$ is projectable (i.e. $\omega=\#_{A}^{*}(\bar{\omega})$ for some $\bar{\omega} \in \Omega^{1}(M)$. On the other hand, if $\omega$ is exact, i.e. $0=[\omega]_{A} \in H^{1}(A)$, then $\nabla^{+}=0$, which impies that the differential equation $\nabla \nu=0$ is locally uniquelly integrable.

By the definition the 0-group of cohomology can be written similarly to (2.1).

$$
H_{\nabla}^{0}(A, \xi)=\{\nu \in \Gamma(\xi) ; \nabla \nu=0\} .
$$

Proposition 7.7. (1) $H_{\nabla}^{0}(A, \xi)=0$ if $\xi$ is nontrivial.

(2) For the trivial vector bundle $\xi=M \times \mathbb{R}$ and $\nabla=\partial_{A}^{\omega}$ for closed 1-form $\omega \in \Omega^{1}(A)$ we have

$$
H_{\nabla}^{0}(A, \xi) \neq 0 \Longleftrightarrow[\omega]_{A}=0 .
$$

In particular, if $H_{\nabla}^{0}(A, \xi) \neq 0$ then $\nabla^{+}=0$. 
Proof. (1) Evidently, since each section of nontrivial line bundle $\xi$ is singular and by Lemma 7.6 the set $\{x ; \nabla \nu=0\}$ is open-closed.

(2) This result may be proved in the same way as in the case of $A=T M$, i.e. as the formula (2.3), see also Example 2.6 from Chapter 1.

Proposition 7.7(1) generalizes observation $(\bullet \bullet)$ from section 2.1.

Proposition 7.8. Let $\xi$ be a line bundle and fix an isomorphism $\varphi: \xi \otimes \xi \rightarrow$ $M \times \mathbb{R}$. Let us assume that $\varphi$ transforms a given A-representation $\nabla: A \rightarrow A(\xi)$ to A-representation $\partial_{A}^{\omega}$ for a closed 1 -form $\omega \in \Omega^{1}(A)$. Then there exists a linear isomorphism

In particular

$$
H_{D^{o r} \otimes \nabla^{-\omega}}^{j}\left(A, Q_{A}^{o r} \otimes \xi\right) \cong H_{\nabla, c}^{m+n-j}(A, \xi)^{*} .
$$

$$
H_{D^{o r} \otimes \nabla^{-\omega}}^{0}\left(A, Q_{A}^{o r} \otimes \xi\right) \cong H_{\nabla, c}^{m+n}(A, \xi)^{*} .
$$

Proof. Consider the multiplication by reals

$$
\rho: Q_{A}^{o r} \times(M \times \mathbb{R}) \rightarrow Q_{A}^{o r} .
$$

$\rho$ is compatible with $\left(D^{o r}, \partial_{A}^{\omega},\left(D^{o r}\right)^{\omega}\right)$. The canonical nondegenerate pairing

$$
F: Q_{A}^{o r} \otimes \xi \times \xi \rightarrow Q_{A}^{o r} \otimes \xi \otimes \xi
$$

is compatible with $\left(D^{o r} \otimes \nabla, \nabla, D^{o r} \otimes \nabla \otimes \nabla\right)$, so the composition

$$
\tilde{F}: Q_{A}^{o r} \otimes \xi \times \xi \stackrel{F}{\rightarrow} Q_{A}^{o r} \otimes \xi \otimes \xi \stackrel{i d \otimes \varphi}{\longrightarrow} Q_{A}^{o r} \otimes(M \times \mathbb{R}) \stackrel{\rho}{\rightarrow} Q_{A}^{o r}
$$

is compatible with $\left(D^{o r} \otimes \nabla, \nabla,\left(D^{o r}\right)^{\omega}\right)$ which implies that it is also compatible with $\left(D^{o r} \otimes \nabla^{-\omega}, \nabla, D^{o r}\right)$. Therefore, for each point $x \in M$, the pairing $\tilde{F}_{x}: Q_{A, x}^{o r} \otimes$ $\xi_{x} \times \xi_{x} \rightarrow Q_{A, x}^{o r}$ is compatible with the representations $\left(\left(D^{o r} \otimes \nabla^{-\omega}\right)_{x}^{+}, \nabla_{x}^{+}, D_{x}^{o r+}\right)$ of the isotropy Lie algebra $\boldsymbol{g}_{x}$ in the vector spaces $Q_{A, x}^{o r} \otimes \xi_{x}, \xi_{x}, Q_{A, x}^{o r}$, respectively. From this it follows that the differentials $d_{\left(D^{o r} \otimes \nabla^{-\omega}\right)_{x}^{+}}, d_{\nabla_{x}^{+}}, d_{\left(D^{o r}\right)_{x}^{+}}$fulfil condition (6) from Lemma 3.1. Of course, $d_{\left(D^{o r}\right)_{x}^{+}}=d_{\text {trad }} \otimes i d$ satisfies condition (3) from the mentioned lemma. Since

$$
\wedge: \Lambda^{i} \boldsymbol{g}_{x}^{*} \otimes\left(Q_{A, x}^{o r} \otimes \xi_{x}\right) \times \Lambda^{n-i} \boldsymbol{g}_{x}^{*} \otimes \xi_{x} \rightarrow \Lambda^{n} \boldsymbol{g}_{x}^{*} \otimes Q_{A, x}^{o r}
$$

is nondegenerate, the generalized Chern-Hirzebruch-Serre Lemma 3.1 asserts that induced pairing in cohomology

$$
H_{\left(D^{o r} \otimes \nabla^{-\omega}\right)^{+}}^{i}\left(\boldsymbol{g}, Q_{A}^{o r} \otimes \xi\right) \times H_{\nabla^{+}}^{n-i}(\boldsymbol{g}, \xi) \rightarrow H_{D^{\text {or+ }}}^{n}\left(\boldsymbol{g}, Q_{A}^{o r}\right) \stackrel{\text { l.(7.2) }}{\rightarrow} \text { or }(M)
$$

is nondegenerate at every point $x \in M$. The fundamental Theorem 4.4, see also Conclusion 6.1, shows that the pairing

$$
H_{D^{o r} \otimes \nabla^{-\omega}}^{j}\left(A, Q_{A}^{o r} \otimes \xi\right) \times H_{\nabla, c}^{m+n-j}(A, \xi) \rightarrow H_{D^{o r}, c}^{m+n}\left(A, Q_{A}^{o r}\right)
$$

is nondegenerate. This ends the proof.

Conclusion 7.9. If $\xi$ is not isomorphic to $Q_{A}^{o r}$ (i.e. $\xi$ is not isomorphic to $\Lambda^{n} \boldsymbol{g} \otimes$ or $(M)$, see (7.1), then for an arbitrary connection $\nabla: A \rightarrow A(\xi)$ we have $H_{\nabla, c}^{m+n}(A, \xi)=0$.

Proof. If $\xi$ is not isomorphic to $Q_{A}^{o r}$ the vector bundle $Q_{A}^{o r} \otimes \xi$ is not trivial so Proposition 7.7 gives $H_{D^{o r} \otimes \nabla^{-\omega}}^{0}\left(A, Q_{A}^{o r} \otimes \xi\right)=0$. Proposition 7.8 proves our theorem. 
The next theorem is one of the importest theorems of the paper. Compare this theorem and Theorem 7.3 with Theorem 5.4 form [H].

Theorem 7.10. For a line bundle $\xi$ and a representation $\nabla: A \rightarrow A(\xi)$ the following conditions are equaivalent:

(a) $H_{\nabla, c}^{m+n}(A, \xi) \neq 0$,

(b) $H_{\nabla, c}^{m+n}(A, \xi)=\mathbb{R}$ and the pairing $H^{j}(A) \times H_{\nabla, c}^{m+n-j}(A, \xi) \rightarrow H_{\nabla, c}^{m+n}(A, \xi) \cong$ $\mathbb{R}$ is nondegenerate, i.e. $H^{j}(A) \cong\left(H_{\nabla, c}^{m+n-j}(A, \xi)\right)^{*}$,

(c) $(\xi, \nabla) \sim\left(Q_{A}^{o r}, D^{o r}\right)$.

Proof. (c) $\Longrightarrow$ (b) by Lemma 7.2, (b) $\Longrightarrow$ (a) is evident.

$(\mathrm{a}) \Longrightarrow(\mathrm{c})$. Let $H_{\nabla, c}^{m+n}(A, \xi) \neq 0$. By Conclusion $7.9 \xi \cong Q_{A}^{o r}$. It remains to compare the representations. Consider then a flat bundle $\left(\xi=Q_{A}^{\text {or }}, \nabla\right)$ and any linear isomorphism

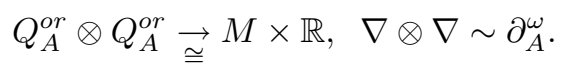

By Proposition 7.8

$$
H_{D^{o r} \otimes \nabla^{-\omega}}^{0}\left(A, Q_{A}^{o r} \otimes Q_{A}^{o r}\right) \cong H_{\nabla, c}^{m+n}\left(A, Q_{A}^{o r}\right) \neq 0,
$$

therefore there exists a nonsingular global section $\nu \in \Gamma\left(Q_{A}^{o r} \otimes Q_{A}^{o r}\right)$ which is $D^{\text {or }} \otimes$ $\nabla^{-\omega}$-constant. Additionally, $\nabla \otimes \nabla \sim \partial_{A}^{\omega}$ implies $\nabla \otimes \nabla^{-\omega} \sim \partial_{A}$ which means that there exists a second nonsingular section $\nu^{\prime} \in \Gamma\left(Q_{A}^{\text {or }} \otimes Q_{A}^{\text {or }}\right)$ which is $\nabla \otimes \nabla^{-\omega_{-}}$ constant. The bundle $Q_{A}^{o r} \otimes Q_{A}^{o r}$ is 1-dimensional, so $\nu^{\prime}=f \cdot \nu$ for a nonsingular function $f \in C^{\infty}(M)$. Write locally $\nu=\nu_{\alpha}^{\prime} \otimes \nu_{\alpha}$ for nonsingular sections $\nu_{\alpha}^{\prime}$, $\nu_{\alpha}$ of $Q_{A}^{o r}$. Then

$$
\begin{aligned}
& 0=\left(D^{o r} \otimes \nabla^{-\omega}\right)_{\gamma}(\nu)=D_{\gamma}^{o r}\left(\nu_{\alpha}^{\prime}\right) \otimes \nu_{\alpha}+\nu_{\alpha}^{\prime} \otimes \nabla_{\gamma}^{-\omega}\left(\nu_{\alpha}\right), \\
& 0=\left(\nabla \otimes \nabla^{-\omega}\right)_{\gamma}(f \cdot \nu)=\nabla_{\gamma}\left(f \cdot \nu_{\alpha}^{\prime}\right) \otimes \nu_{\alpha}+f \cdot \nu_{\alpha}^{\prime} \otimes \nabla_{\gamma}^{-\omega}\left(\nu_{\alpha}\right) .
\end{aligned}
$$

Multiplying first equation by $f$ and then substracting the second we get

$$
\left(f \cdot D_{\gamma}^{o r}\left(\nu_{\alpha}^{\prime}\right)-\nabla_{\gamma}\left(f \cdot \nu_{\alpha}^{\prime}\right)\right) \otimes \nu_{\alpha}=0
$$

The nonsingularity of $\nu_{\alpha}$ yields the equation $f \cdot D_{\gamma}^{o r}\left(\nu_{\alpha}^{\prime}\right)=\nabla_{\gamma}\left(f \cdot \nu_{\alpha}^{\prime}\right)$. The bundle $Q_{A}^{\text {or }}$ is 1-dimensional, so

$$
f \cdot D_{\gamma}^{o r}(\bar{\nu})=\nabla_{\gamma}(f \cdot \bar{\nu})
$$

for all $\bar{\nu} \in \Gamma\left(Q_{A}^{o r}\right)$. Define a linear isomorphism

$$
\varphi: Q_{A}^{o r} \rightarrow Q_{A}^{o r}, \quad \bar{\nu} \mapsto f \cdot \bar{\nu}
$$

By (7.4) one has that $\left(Q_{A}^{o r}, \nabla\right) \sim\left(Q_{A}^{o r}, D^{o r}\right)$.

7.4. Characterization of transitive Lie algebroids with Poincaré duality. The last aim is to characterize two classes of transitive Lie algebroids.

(1) $H_{\partial_{A}, c}^{m+n}(A) \neq 0$ - the top group of real compact cohomology is not trivial.

This condition is equivalent to $\left(Q_{A}^{o r}, D^{\text {or }}\right) \sim\left(M \times \mathbb{R}, \partial_{A}\right)$. These classes fulfil the Poincaré duality: the pairing

$$
H^{j}(A) \times H_{c}^{m+n-j}(A) \rightarrow H_{c}^{m+n}(A) \cong \mathbb{R}
$$

is not degenerate, see Theorem 7.3, i.e. $H^{j}(A) \cong\left(H_{c}^{m+n-j}(A)\right)^{*}$. 
(2) $H_{\partial_{A}^{\circ r}, c}^{m+n}(A$, or $(M)) \neq 0$ - the top group of or $(M)$-valued compact cohomology is not trivial.

This condition is equivalent to $\left(Q_{A}^{o r}, D^{o r}\right) \sim\left(\operatorname{or}(M), \partial_{A}^{o r}\right)$. In this class the multiplication of cohomology classes

$$
H^{j}(A) \times H_{\partial_{A}^{o r}, c}^{m+n-j}(A, \text { or }(M)) \rightarrow H_{\partial_{A}^{o r}, c}^{m+n}(A, \text { or }(M)) \cong \mathbb{R}
$$

is not degenerate, see Theorem 7.3, i.e. $H^{j}(A) \cong\left(H_{\partial_{A}^{o r}, c}^{m+n-j}(A)\right)^{*}$.

Before the characterization of these classes we reduce the Evens-Lu-Weinstein representation $\left(Q_{A}^{o r}, D^{o r}\right)$ to equivalent simple form (only for transitive Lie algebroids of course). We recall that the adjoint representation $a d_{A}: A \rightarrow A(\boldsymbol{g})$ induces a top-power representaion $a d_{A}^{\text {top }}: A \rightarrow A\left(\Lambda^{\text {top }} \boldsymbol{g}\right)$ by

$$
\left(a d_{A}^{\text {top }}\right)_{\gamma}\left(\sigma_{1} \wedge \ldots \wedge \sigma_{n}\right)=\sum_{i} \sigma_{1} \wedge \ldots \wedge \llbracket \gamma, \sigma_{i} \rrbracket \wedge \ldots \wedge \sigma_{n}=\sum_{i} a_{i}^{i} \cdot \sigma_{1} \wedge \ldots \wedge \sigma_{n}
$$

where $\llbracket \gamma, \sigma_{i} \rrbracket=\sum_{j} a_{i}^{j} \cdot \sigma_{j}$.

Lemma 7.11. There exist isomorphisms of flat vector bundles

$$
\begin{aligned}
\left(Q_{A}, D\right) & \cong\left(\Lambda^{\mathrm{top}} \boldsymbol{g}, a d_{A}^{\mathrm{top}}\right), \\
\left(Q_{A}^{o r}, D^{o r}\right) & \cong\left(\Lambda^{\mathrm{top}} \boldsymbol{g} \otimes \text { or }(M), a d_{A}^{\mathrm{top}} \otimes \partial_{A}^{o r}\right) .
\end{aligned}
$$

Proof. It is necessary to show the first assertion, because the second follows from first by tensor product with or $(M)$. Fix arbitrary a connection $\lambda: T M \rightarrow A$ and a linear isomorphism

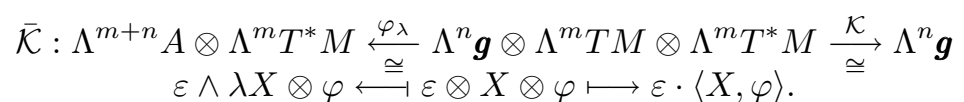

Taking a local basis $\sigma_{1}, \ldots, \sigma_{n}$ of $\boldsymbol{g}, W_{1}, \ldots, W_{m}$ of $T M$ and the duals $W_{1}^{*}, \ldots, W_{m}^{*}$ we see that $\overline{\mathcal{K}}\left(\sigma_{1} \wedge \ldots \wedge \sigma_{n} \wedge \lambda W_{1} \wedge \ldots \wedge \lambda W_{m} \otimes W_{1}^{*} \wedge \ldots \wedge W_{m}^{*}\right)=\sigma_{1} \wedge \ldots \wedge \sigma_{n}$. To prove our lemma it is necessary to show the compatibility $D \stackrel{\overline{\mathcal{K}}}{\sim} a d_{A}^{n}$ on these nonsingular sections only, i.e.

$$
\left(a d_{A}^{n}\right)_{\gamma}\left(\sigma_{1} \wedge \ldots \wedge \sigma_{n}\right)=\overline{\mathcal{K}}\left(D_{\gamma}\left(\sigma_{1} \wedge \ldots \wedge \sigma_{n} \wedge \lambda W_{1} \wedge \ldots \wedge \lambda W_{m} \otimes W_{1}^{*} \wedge \ldots \wedge W_{m}^{*}\right)\right)
$$

Let $\llbracket \gamma, \sigma_{i} \rrbracket=\sum_{j} a_{i}^{j} \cdot \sigma_{j}, \llbracket \gamma, \lambda W_{j} \rrbracket=\sum_{k} \tilde{a}_{j}^{k} \cdot \sigma_{k}+\sum_{r} b_{j}^{r} \cdot \lambda W_{r}$, then $\left[\# A_{A}(\gamma), W_{j}\right]=$ $\sum_{r} b_{j}^{r} \cdot \lambda W_{r}$. The right side of the above equation is equal to

$$
\begin{aligned}
& \overline{\mathcal{K}}\left(\sum a_{i}^{i} \cdot \sigma_{1} \wedge \ldots \wedge \sigma_{n} \wedge \lambda W_{1} \wedge \ldots \wedge \lambda W_{m} \otimes W_{1}^{*} \wedge \ldots \wedge W_{m}^{*}+\right. \\
& +\sigma_{1} \wedge \ldots \wedge \sigma_{n} \wedge \sum b_{j}^{j} \wedge \lambda W_{1} \wedge \ldots \wedge \lambda W_{m} \otimes W_{1}^{*} \wedge \ldots \wedge W_{m}^{*}+ \\
& \left.+\sigma_{1} \wedge \ldots \wedge \sigma_{n} \wedge \lambda W_{1} \wedge \ldots \wedge \lambda W_{m} \otimes\left(-\sum b_{j}^{j}\right) W_{1}^{*} \wedge \ldots \wedge W_{m}^{*}\right) \\
& =\overline{\mathcal{K}}\left(\sum a_{i}^{i} \cdot \sigma_{1} \wedge \ldots \wedge \sigma_{n} \wedge \lambda W_{1} \wedge \ldots \wedge \lambda W_{m} \otimes W_{1}^{*} \wedge \ldots \wedge W_{m}^{*}\right) \\
& =\sum a_{i}^{i} \cdot\left(\overline{\mathcal{K}}\left(\sigma_{1} \wedge \ldots \wedge \sigma_{n} \wedge \lambda W_{1} \wedge \ldots \wedge \lambda W_{m} \otimes W_{1}^{*} \wedge \ldots \wedge W_{m}^{*}\right)\right) \\
& =\left(a d_{A}^{n}\right)_{\gamma} \cdot \sigma_{1} \wedge \ldots \wedge \sigma_{n} .
\end{aligned}
$$




\section{Conclusion 7.12.}

(1)

$$
H_{\partial_{A}, c}^{m+n}(A) \neq 0 \quad \Longleftrightarrow \quad\left(\Lambda^{n} \boldsymbol{g} \otimes \operatorname{or}(M), a d_{A}^{n} \otimes \partial_{A}^{o r}\right) \sim\left(M \times \mathbb{R}, \partial_{A}\right),
$$

$$
H_{\partial_{A}^{o r}, c}^{m+n}(A, \text { or }(M)) \neq 0 \quad \Longleftrightarrow \quad\left(\Lambda^{n} \boldsymbol{g} \otimes \text { or }(M), a d_{A}^{n} \otimes \partial_{A}^{o r}\right) \sim\left(\text { or }(M), \partial_{A}^{o r}\right) .
$$

The following proposition generalizes Proposition 2.2. The proof is analogous.

Proposition 7.13. For a representation $\nabla: A \rightarrow A(\xi)$ in a line vector bundle $\xi$ the following conditions are equivalent:

(a) $(\xi, \nabla) \sim\left(\right.$ or $\left.(M), \partial_{A}^{o r}\right)$,

(b) $\left(\xi \otimes\right.$ or $\left.(M), \nabla \otimes \partial_{A}^{o r}\right) \sim\left(M \times \mathbb{R}, \partial_{A}\right)$.

In the sequel we need the notion of a modular class of a Lie algebroid [W], [E-L-W]. Firstly, we recall the characteristic classes of a representation $\nabla: A \rightarrow$ $A(\xi)$ in a line vector bundle $\xi$. If $\xi$ is trivial as a line bundle and $s \in \Gamma(\xi)$ is a nonsingular section of $\xi$ we define a 1-cocycle $\theta \in \Omega^{1}(A)$ with respect to $d_{A}$ defined by $\nabla_{\gamma} \nu=\theta_{s}(\gamma) \cdot s$. The class $\theta_{\nabla}=[\theta] \in H^{1}(A)$ is independent on the choice of $s$ and is called characteristic class of $A$ associated to the representation $\nabla$. For a general $\xi$, we define $\theta_{\nabla}=\frac{1}{2} \theta_{\nabla \otimes \nabla}(\nabla \otimes \nabla$ is a flat representation in trivial line bundle $\xi \otimes \xi$ ). We add that if $\xi$ is trivial, the last equation holds.

For next propositions and theorems we need the following lemma.

Lemma 7.14. If $\xi$ is a line bundle and $\left\{\varphi_{\alpha}\right\}$ is a collection of local trivialiations with the transition functions $\lambda_{\alpha \beta}: U_{\alpha} \times U_{\beta} \rightarrow \mathbb{R}, \varphi_{\beta}=\varphi_{\alpha} \cdot \lambda_{\alpha \beta}$, then there exist functions $f_{\alpha}>0$ such that the local trivializations

$$
\bar{\varphi}_{\alpha}=\varphi_{\alpha} \cdot f_{\alpha}
$$

$\left(\bar{\varphi}_{\alpha, x}=\varphi_{\alpha, x} \cdot f_{\alpha}(x)\right)$ have transition functions $\bar{\lambda}_{\alpha \beta}=\operatorname{sgn} \lambda_{\alpha \beta}$.

In conclusion, each line bundle $\xi$ possesses a system of local trivializations with transition functions equaling to \pm 1 and then a family $\left\{s_{\alpha}\right\}$ of nonsingular \pm 1 sections i.e. with transition functions equaling just to \pm 1 .

Proof. Consider a line bundle $\xi$ with a collection of local trivialiations $\left\{\varphi_{\alpha}\right\}$ and transition functions $\lambda_{\alpha \beta}$. The tensor product $\xi \otimes \xi$ is a trivializable vector bundle with local system of trivializations $\left\{\varphi_{\alpha} \otimes \varphi_{\alpha}\right\}$. Choice a global trivialization $\rho$ : $\xi \otimes \xi \rightarrow M \times \mathbb{R}$ such that $\rho_{\alpha}:=\rho\left(\varphi_{\alpha} \otimes \varphi_{\alpha}(1 \otimes 1)\right)>0$. We put

$$
f_{\alpha}=\frac{1}{\sqrt{\rho_{\alpha}}}>0 \text {. }
$$

We show that $\left\{f_{\alpha}\right\}$ is a required family. The transition functions $\bar{\lambda}_{\alpha \beta}$ for the collection of new local trivializations $\left\{\bar{\varphi}_{\alpha}:=\varphi_{\alpha} \cdot f_{\alpha}\right\}$ are equl to $\bar{\lambda}_{\alpha \beta}=\lambda_{\alpha \beta} \cdot \frac{f_{\beta}}{f_{\alpha}}$ so that $\operatorname{sgn} \bar{\lambda}_{\alpha \beta}=\operatorname{sgn} \lambda_{\alpha \beta}$. On the other hand, $\rho_{\beta}=\rho\left(\varphi_{\beta} \otimes \varphi_{\beta}(1 \otimes 1)\right)=\lambda_{\alpha \beta}^{2} \cdot \rho_{\alpha}$ so $\bar{\lambda}_{\alpha \beta}^{2}=\left(\lambda_{\alpha \beta} \cdot \frac{f_{\beta}}{f_{\alpha}}\right)^{2}=\lambda_{\alpha \beta}^{2} \cdot \frac{\rho_{a}}{\rho_{\beta}}=1$ and next $\left|\bar{\lambda}_{\alpha \beta}\right|=1$. Finally $\bar{\lambda}_{\alpha \beta}=\operatorname{sgn} \bar{\lambda}_{\alpha \beta}=$ $\operatorname{sgn} \lambda_{\alpha \beta}$.

Lemma 7.15. For an arbitrary line bundle $\xi$ the characteristic class $\theta_{\nabla}$ of a representation $\nabla: A \rightarrow A(\xi)$ can be computed via any family of local nonsingular \pm sections $\left\{s_{\alpha}\right\}$ (see Lemma 7.14) of $\xi$ in the following way: the 1-differential $A$ form $\theta \in \Omega^{1}(A)$ defined by

$$
\theta(\gamma)_{\mid U_{\alpha}} s_{\alpha}=\nabla_{\gamma}\left(s_{\alpha}\right)
$$


is a correctly defined $d_{A}$-cocycle and its cohomology class is equal to $\theta_{\nabla} \cdot \theta_{\nabla}=0$ if and only if there exists a family of local nonsingular $\nabla$-constant \pm sections $\left\{s_{\alpha}\right\}$, $\nabla s_{\alpha}=0$. For transitive Lie algebroid $A$ if $\theta_{\nabla}=0$ then $\nabla^{+}=0$, so the isotropy Lie algebras $\boldsymbol{g}_{x}$ are unimodular.

The simple proof will be omitted.

We have $\theta_{\partial_{A}^{o r}}=0$. The modular class of a Lie algebroid $A$ is by definition the characteristic class $\theta_{A}$ of the associated representation $D: A \rightarrow A\left(Q_{A}\right)$. According to Lemma 7.11 for a transitive Lia algebroid $A$ we have $\theta_{A}=\theta_{a d_{A}^{n}}$ where $a d_{A}^{n}$ : $A \rightarrow A\left(\Lambda^{n} \boldsymbol{g}\right)$ is the representation induced by the adjoint one $a d_{A}: A \rightarrow A(\boldsymbol{g})$.

For real coefficients we have the following characterization of Lie algebroids in the case of the nontriviality of the top group of cohomology. Let $\left\{\left(U_{\alpha}, x_{a}\right)\right\}$ be a coordinate open cover for the manifold $M$, with transition functions $g_{\alpha \beta}=x_{\alpha} \circ x_{\beta}^{-1}$. Each map $x_{a}$ determines canonically a local trivialization $\bar{x}_{\alpha}$ of the line bundle $\Lambda^{m} T M$ and the family $\left\{\bar{x}_{\alpha}\right\}$ has the transition functions $J\left(g_{\alpha \beta}\right)$.

Theorem 7.16. The following conditions are equivalent

(a) $H_{\partial_{A}, c}^{m+n}(A) \neq 0$,

(b) $H_{\partial_{A}, c}^{m+n}(A) \cong \mathbb{R}$ and $H(A)$ is an Poincaré algebra, i.e. the pairing $H^{j}(A) \times$ $H_{c}^{m+n-j}(A) \rightarrow H_{c}^{m+n}(A) \cong \mathbb{R}$ is nondegenerate, $H^{j}(A) \cong\left(H_{c}^{m+n-j}(A)\right)^{*}$,

(c) $\left(Q_{A}^{o r}, D^{o r}\right) \sim\left(M \times \mathbb{R}, \partial_{A}\right)$,

(d) $\left(\Lambda^{n} \boldsymbol{g} \otimes\right.$ or $\left.(M), a d_{A}^{n} \otimes \partial_{A}^{o r}\right) \sim\left(M \times \mathbb{R}, \partial_{A}\right)$,

(e) $\left(\Lambda^{n} \boldsymbol{g}, a d_{A}^{n}\right) \sim\left(\right.$ or $\left.(M), \partial_{A}^{o r}\right)$, that is the holonomy homomorphism of $\left(\Lambda^{n} \boldsymbol{g}, a d_{A}^{n}\right)$ is the same as for the orientation bundle (or $\left.(M), \partial_{A}^{o r}\right)$.

(f) $A$ is orientable vector bundle and $\theta_{A}=0$. (in particular, $\boldsymbol{g}_{x}$ are unimodular).

Proof. (a) $\Longleftrightarrow$ (b) $\Longleftrightarrow$ (c) follows immediately from Theorem 7.10 for $(\xi, \nabla)=$ $\left(M \times \mathbb{R}, \partial_{A}\right)$,

(c) $\Longleftrightarrow(d)$ by Lemma 7.11,

(d) $\Longleftrightarrow$ (e) see Proposition 7.13,

(e) $\Longrightarrow$ (f) indeed, $\theta_{A}=\theta_{a d_{A}^{n}}=\theta_{\partial_{A}^{o r}}=0$. The bundle $\Lambda^{m+n} A \cong \Lambda^{n} \boldsymbol{g} \otimes \Lambda^{m} T M \cong$ or $(M) \otimes \Lambda^{m} T M$ is trivial line bundle because it possesses a local system of trivializations with positive transition functions $\left|J\left(g_{\alpha \beta}\right)\right|$.

(f) $\Longrightarrow$ (a) It is necessary to find a local system of nonsingular $a d_{A}^{n}$-constant sections $\left\{\sigma_{\alpha}\right\}$ of $\Lambda^{n} \boldsymbol{g}$ with transition functions $\operatorname{sgn} J\left(g_{\alpha \beta}\right)$.

Fix a system of local trivializations $\left\{\psi_{\alpha}\right\}$ of $\Lambda^{m+n} A$ with positive transition functions $\gamma_{\alpha \beta}>0$. We can choose a system of local trivializations $\left\{\varphi_{\alpha}\right\}$ of the line bundle $\Lambda^{n} \boldsymbol{g}$ in such a way that $\varphi_{\alpha} \otimes \bar{x}_{\alpha}$ form a system of local trivializations of the line bundle $\Lambda^{m+n} A \cong \Lambda^{n} \boldsymbol{g} \otimes \Lambda^{m} T M$ compatible with $\left\{\psi_{\alpha}\right\}$, i.e. such that

$$
\varphi_{\alpha} \otimes \bar{x}_{\alpha}=g_{\alpha} \cdot \psi_{\alpha}, g_{\alpha}>0 .
$$

This implies that the transition functions $\lambda_{\alpha \beta}$ of the system $\left\{\varphi_{\alpha}\right\}$ have the sign of $J\left(g_{\alpha \beta}\right)$. Indeed,

$$
\varphi_{\beta} \otimes \bar{x}_{\beta}=\left\{\begin{array}{l}
\varphi_{\alpha} \otimes \bar{x}_{\alpha} \cdot \lambda_{\alpha \beta} \cdot J\left(g_{\alpha \beta}\right)=\psi_{\alpha} \cdot g_{\alpha} \cdot \lambda_{\alpha \beta} \cdot J\left(g_{\alpha \beta}\right), \\
\psi_{\beta} \cdot g_{\beta}=\psi_{\alpha} \cdot \gamma_{\alpha \beta} \cdot g_{\beta} .
\end{array}\right.
$$

Therefore, since $g_{\alpha}, g_{\beta}$ and $\gamma_{\alpha \beta}$ are positive we have $\lambda_{\alpha \beta} \cdot J\left(g_{\alpha \beta}\right)>0$, i.e.

$$
\operatorname{sgn} \lambda_{\alpha \beta}=\operatorname{sgn} J\left(g_{\alpha \beta}\right) .
$$


By Lemma 7.14 there exists functions $f_{\alpha}>0$ such that the local trivializations

$$
\bar{\varphi}_{\alpha}=\varphi_{\alpha} \cdot f_{\alpha}
$$

$\left(\bar{\varphi}_{\alpha, x}=\varphi_{\alpha, x} \cdot f_{\alpha}(x)\right)$ have transition functions $\bar{\lambda}_{\alpha \beta}=\operatorname{sgn} \lambda_{\alpha \beta}=\operatorname{sgn} J\left(g_{\alpha \beta}\right)$. The family of \pm sections $\bar{\sigma}_{\alpha}=\bar{\varphi}_{\alpha}(1)$ determine a 1-cocycle $\theta \in \Omega^{1}(A)$ with respect $\operatorname{do} d_{A}$ defined by $\theta(\gamma)_{U_{\alpha}} \cdot \bar{\sigma}_{\alpha}=\left(a d_{A}^{n}\right)_{\gamma}\left(\bar{\sigma}_{\alpha}\right)$ whose cohomology class is the characteristic class of the adjoint representation $a d_{A}^{n},[\theta]=\theta_{a d_{A}^{n}}$. Since $\theta_{a d_{A}^{n}}=\theta_{A}=0$ one has $\theta=d_{A} f$ for some function $f \in C^{\infty}(M)$, i.e.

$$
\theta(\gamma)=\left(d_{A} f\right)(\gamma)=\partial_{\#_{A}(\gamma)} f
$$

Put $\sigma_{\alpha}=e^{-f} \cdot \bar{\sigma}_{\alpha}$. Then the transition functions of $\left\{\sigma_{\alpha}\right\}$ are equal to $\operatorname{sgn} J\left(g_{\alpha \beta}\right)$ and the sections $\sigma_{\alpha}$ are $a d_{A}^{n}$-constant.

In case of oriented manifold the above theorem yields:

Theorem 7.17. If $M$ is a oriented manifold then the following conditions are equivalent

(a) $H_{\partial_{A}, c}^{m+n}(A) \neq 0$

(b) $H_{\partial_{A}, c}^{m+n}(A) \cong \mathbb{R}$ and $H(A)$ is an Poincaré algebra, i.e. the pairing $H^{j}(A) \times$ $H_{c}^{m+n-j}(A) \rightarrow H_{c}^{m+n}(A) \cong \mathbb{R}$ is nondegenerate, $H^{j}(A) \cong\left(H_{c}^{m+n-j}(A)\right)^{*}$,

(c) $\left(\Lambda^{n} \boldsymbol{g}, a d_{A}^{n}\right) \sim\left(M \times \mathbb{R}, \partial_{A}\right)$, i.e. there exists a global nonsingular section $\varepsilon \in \Gamma\left(\Lambda^{n} \boldsymbol{g}\right)$ which is ad ${ }_{A}^{n}$-constant, that is, A is a TUIO-Lie algebroid, see [K2],

(d) $\boldsymbol{g}$ is orientable and $\theta_{A}=0$.

The independent proof of the implication $(\mathrm{c}) \Longrightarrow(\mathrm{b})$ one can be found in [K2].

Finally we give a characterization of Lie algebroids whose the top group of cohomology with coefficients in the orientation bundle or $(M)$ is not trivial.

Theorem 7.18. The following conditions are equivalent:

(a) $H_{\partial_{A}^{o r}, c}^{m+n}(A$, or $(M)) \neq 0$,

(b) $H_{\partial_{A}^{o r}, c}^{m+n}(A$, or $(M)) \cong \mathbb{R}$ and the pairing

$$
H^{j}(A) \times H_{\partial_{A}^{o r}, c}^{m+n-j}(A, \text { or }(M)) \rightarrow H_{\partial_{A}^{o r}, c}^{m+n}(A, \text { or }(M)) \cong \mathbb{R}
$$

is not degenerate, i.e. $H^{j}(A) \cong\left(H_{\partial_{A}^{o r}, c}^{m+n-j}(A, \text { or }(M))\right)^{*}$,

(c) $\left(Q_{A}^{o r}, D^{o r}\right) \sim\left(\right.$ or $\left.(M), \partial_{A}^{o r}\right)$,

(d) $\left(\Lambda^{n} \boldsymbol{g} \otimes\right.$ or $\left.(M), a d_{A}^{n} \otimes \partial_{A}^{o r}\right) \sim\left(\right.$ or $\left.(M), \partial_{A}^{o r}\right)$,

(e) $\left(\Lambda^{n} \boldsymbol{g}, a d_{A}^{n}\right) \sim\left(M \times \mathbb{R}, \partial_{A}\right)$,

(f) $\boldsymbol{g}$ is orientable and there exists a global nonsingular section $\varepsilon \in \Gamma(\xi)$ which is $\operatorname{ad}_{A}^{n}$-constant (i.e. $A$ is a TUIO-Lie algebroid, see [K2]),

(g) $\boldsymbol{g}$ is orientable and $\theta_{A}=0$.

Proof. Only the implication $(\mathrm{d}) \Longrightarrow(\mathrm{e})$ needs a proof. Since

$$
\left(\text { or }(M) \otimes \text { or }(M), \partial_{A}^{\text {or }} \otimes \partial_{A}^{\text {or }}\right) \sim\left(M \times \mathbb{R}, \partial_{A}\right)
$$

one has

$$
\begin{aligned}
\left(\Lambda^{n} \boldsymbol{g}, a d_{A}^{n}\right) & \sim\left(\Lambda^{n} \boldsymbol{g} \otimes \text { or }(M) \otimes \text { or }(M), a d_{A}^{n} \otimes \partial_{A}^{\text {or }} \otimes \partial_{A}^{o r}\right) \\
& \stackrel{(\mathrm{d})}{\sim}\left(\operatorname{or}(M) \otimes \operatorname{or}(M), \partial_{A}^{\text {or }} \otimes \partial_{A}^{o r}\right) \\
& \sim\left(M \times \mathbb{R}, \partial_{A}\right) .
\end{aligned}
$$


For an orientable manifold we get Conclusion 7.17.

7.5. Remarks on Example 5.3 from [E-L-W]. In the cited paper there is an example of nonregular Lie algebroid for which the E-L-W cohomological pairing is not necessary nondegenerate. In the text of Example 5.3 from [E-L-W] there are some inaccuracies which we remove here. We prove additionally that there is no line representation for which the cohomological pairing is nondegenerate and we prove that the E-L-W representation is not exceptional. The example is the Lie transformation algebroid $A=\mathfrak{g} \times M \rightarrow M$ associated with the infinitesimal action $\gamma: \mathfrak{g} \rightarrow \mathfrak{X}(M)$ of a finitely dimensional Lie algebra $\mathfrak{g}$ on a manifold $M$. The anchor is given by $\rho(v, x)=\gamma(v)_{x}$, and Lie bracket by

$$
\llbracket a, b \rrbracket(x)=[a(x), b(x)]+\gamma(a(x))_{x}(b)-\gamma(b(x))_{x}(\eta),
$$

$a, b \in C^{\infty}(M, \mathfrak{g}) \cong \Gamma(\mathfrak{g} \times M)$ and $x \in M$. The vector field $X=x^{N} \frac{d}{d x}$ on $\mathbb{R}$ $(N \in \mathbb{N})$ defines an action of the 1-dimensional Lie algebra $\mathfrak{g}=\mathbb{R}$ on $M=\mathbb{R}$ by $\gamma: \mathbb{R} \rightarrow \mathfrak{X}(\mathbb{R}), \gamma(t)=t \cdot X$. Let $A$ be the transformation Lie algebroid associated with $\gamma$. Then $\Gamma(A)=C^{\infty}(\mathbb{R}), \#_{x}: A_{x}=\mathbb{R} \rightarrow T_{x} M, t \mapsto t \cdot x^{N} \cdot \frac{d}{d x}, \llbracket a, b \rrbracket=$ $x^{N} \cdot\left(a \cdot b^{\prime}-b \cdot a^{\prime}\right), \Omega^{0}(A)=C^{\infty}(\mathbb{R})$,

$$
\Omega^{1}(A)=\Gamma\left(A^{*}\right)=C^{\infty}\left(\mathbb{R}, \mathbb{R}^{*}\right)=\Omega^{1}(\mathbb{R}) \cong C^{\infty}(\mathbb{R}), f d x \mapsto f,
$$

and, clearly, $\Omega^{2}(A)=0$.

Lemma 7.19. $H^{1}(A) \cong \mathbb{R}^{N}$.

Proof. By definition $d_{A}: C^{\infty}(\mathbb{R}) \rightarrow \Omega^{1}(A) \cong C^{\infty}(\mathbb{R}), d_{A}(f)(a)=\#(a)(f)=$ $a \cdot x^{N} \cdot f^{\prime}$, and therefore $d_{A}(f)=x^{N} \cdot f^{\prime}$ and

$$
H^{1}(A)=C^{\infty}(\mathbb{R})_{/\left\{x^{N} \cdot f^{\prime}, f \in C^{\infty}(\mathbb{R})\right\}}=C^{\infty}(\mathbb{R})_{/ x^{N} \cdot C^{\infty}(\mathbb{R})} \cong \mathbb{R}^{N} .
$$

Indeed, the classes of functions $x^{0}, x^{1}, \ldots, x^{N-1}$ form a basis of $C^{\infty}(\mathbb{R}) / x^{N} \cdot C^{\infty}(\mathbb{R})$ because the classes are linearly independent and for any $f \in C^{\infty}(\mathbb{R})$ the equality $[f]=\sum_{i=0}^{N-1} a_{k}\left[x^{k}\right]$ holds where $a_{k}=\frac{f^{(k)}(0)}{k !}$.

Proposition 7.20. For each linear representation $\nabla: A \rightarrow A(\xi)$ we have (1) $H_{\nabla, c}^{0}(A, \xi)=0$, (2) $H_{\nabla, c}^{1}(A, \xi) \neq 0$.

Therefore, for each representation $\nabla$ of $A$ in a line bundle $\xi$ the cohomological pairing $H^{1}(A) \times H_{\nabla, c}^{0}(A, \xi) \rightarrow H_{\nabla, c}^{1}(A, \xi)$ is not nondegenerate even in a weak manner ${ }^{1}$.

Proof. (1): The line bundle $\xi$ over $\mathbb{R}$ is trivial $\xi=M \times \mathbb{R}(M=\mathbb{R})$ so each representation $\nabla: A \rightarrow A(\xi)$ is of the form $\nabla=\partial_{A}^{\omega}$ for some 1-form $\omega \in \Omega^{1}(A)$. Let $\omega(a)=g \cdot a$ for $g \in C^{\infty}(\mathbb{R})$. Then $\left(\partial_{A}^{\omega}\right)_{a}(f)=\left(\partial_{A}\right)_{a}(f)+\omega(a) \cdot f=a \cdot x^{N} \cdot f^{\prime}+a \cdot g \cdot f$ and

$$
H_{\nabla, c}^{0}(A, \xi)=\left\{f \in C_{c}^{\infty}(\mathbb{R}) ; x^{N} \cdot f^{\prime}+g \cdot f=0\right\}=0 .
$$

by the uniqueness of the Cauchy problem for the differential equation $y^{\prime}=-\frac{g(x)}{x^{N}} \cdot y$.

\footnotetext{
${ }^{1}$ A pairing $F: V \times W \rightarrow U$ is called weakly non-degenerated if both null spaces $N_{1}=$ $\{v \in V ; F(v, \cdot)\}$ and $N_{2}=\{w \in W ; F(\cdot, w)\}$ are zero.
} 
(2) $H_{\nabla, c}^{1}(A, \xi)=C_{c}^{\infty}(\mathbb{R})_{/\left\{x^{N} \cdot f^{\prime}+g \cdot f ; f \in C_{c}^{\infty}(\mathbb{R})\right\}} \neq 0$. To prove this we find a compactly supported function $h \in C_{c}^{\infty}(\mathbb{R})$. such that the differential equation

$$
x^{N} \cdot y^{\prime}+g \cdot y=h
$$

has no global solution $y \in C_{c}^{\infty}(\mathbb{R})$.

Case $g(0)=0$. For any $h$ such that $h(0) \neq 0$ there is no solution of (7.6).

Case $g(0) \neq 0$. Let $|g(x)| \geq \delta>0$ for $|x| \leq \varepsilon, \varepsilon>0$. Take any function $h \in C_{c}^{\infty}(\mathbb{R})$ such that $h \geq 0, h \neq 0$ and $\operatorname{supp} h \subset[\alpha, \beta] \subset(\varepsilon, \infty)$. The elementary theory of linear differential equations [the formula solving the Cauchy problem in the form of denoted integrals] yields easily that no global compactly supported solution of (7.6) exists.

Consider the E-L-W representation $D: A \rightarrow A\left(Q_{A}\right)$. We see that $Q_{A}=A \otimes$ $T^{*} \mathbb{R} \cong M \times \mathbb{R}[M=\mathbb{R}]$ so $\Gamma\left(Q_{A}\right)=\Gamma(A) \otimes \Omega^{1}(\mathbb{R}) \cong C^{\infty}(M)$ by $1 \otimes f d x \mapsto f$ and that $D$ is equivalent to $\partial_{A}^{\omega}$ for $\omega \cong\left(x^{N}\right)^{\prime}$ (with respect to isomorphism (7.5)). According to Proposition (7.20) the top group of cohomology of $A$ for trivial and for E-L-W representations are nontrivial. We prove that this representations are not isomorphic so the E-L-W representation is not exceptional.

Proposition 7.21. The A-flat line bundles $\left(M \times \mathbb{R}, \partial_{A}\right)$ and $\left(Q_{A}, D\right)[M=\mathbb{R}]$ are not isomorphic.

Proof. Let $\varphi: M \times \mathbb{R} \rightarrow Q_{A}$ be a linear homomorphism compatible with $\partial_{A}$ and $D$. $\varphi$ is of the form $\varphi(f)=1 \otimes g \cdot f \cdot d x$ for some $g \in C^{\infty}(M)$. The equality $D_{a}(\varphi(f))=\varphi\left(\left(\partial_{A}\right)_{a} f\right)$ yields $a \cdot\left(x^{N} \cdot f g\right)^{\prime}=a \cdot x^{N} \cdot f^{\prime} \cdot g$, therefore $\left(x^{N} \cdot g\right)^{\prime}=0$ which produces $g \equiv 0$ and that $\varphi$ is not an isomorphism.

\section{REFERENCES}

[B-T] R. Bott, L. Tu, Differential forms in algebraic topology, GTM 82, Springer-Verlag 1982.

[Ch-H-S] S.S.Chern, F.Hirzebruch, J-P. Serre, On the index of a fibered manifold, Proc. AMS, 8 (1957), 587-596.

[C] M. Crainic, Differentiable and algebroid cohomology, Van Est isomorphisms, and characteristic classes, preprint, arXiv:math.DG/0008064, Commentarii Mathematici Helvetici, to appear.

[E-L-W] S.Evens, J-H.Lu, and A.Weinstein, Transverse measures, the modular class and a cohomology pairing for Lie algebroids, Quart. J. Math. Oxford 50 (1999), 417-436.

[F] R. L. Fernandes, Lie algebroids, holonomy and characteristic classes, preprint DG/007132, Advances in Mathematics 170, (2002) 119-179.

[G-L] F. Guedira, A. Lichnerowicz, Géometrie des algébres de Lie locales de Kirillov,J. Math. Pures Appl. 63 (1984), 407-484.

[G-H-V] W. Greub, S. Halperin, R. Vanstone, Connections, Curvature, and Cohomology, Vol. I, 1971, Vol.II 1973, New York and London.

[H-R] S. Haller, T. Rybicki, Reduction for locally conformal symplectic manifolds, J. Geom. Phys. 37 (2001), 262-271.

[H-S] G.Hochschild, and J.-P.Serre, Cohomology of Lie algebras, Ann. Math. 57, 1953, 591603.

[H] J.Huebschmann, Duality for Lie-Rinehart algebras and the modular class, J.Reine Angew: Math 510 (1999), 103-159.

[I-K-V] V.Itskov, M.Karashev, and Y.Vorobjev, Infinitesimal Poisson Cohomology, Amer. Math. Soc. Transl. (2) Vol. 187, 1998.

[K1] J.Kubarski, Invariant cohomology of regular Lie algebroids, Proceedings of the VII International Colloquium on Differential Geometry ANALYSIS AND GEOMETRY IN FOLIATED MANIFOLDS, Santiago de Compostela, Spain, July 1994, ed. by X.Masa, E. Macias-Virgos, J. Alvarez Lopez; World Scientific, Singapure 1995, pp. 137-151. 
[K2] J.Kubarski, Fibre integral in regular Lie algebroids, New Developments in Differential Geometry, Budapest 1996, Proceedings of the Conference on Differential Geometry, Budapest, Hungary, July 27-30, 1996; Kluwer Academic Publishers 1999.

[K3] J.Kubarski, Poincaré duality for transitive unimodular invariantly oriented Lie algebroids, Topology and Its Applications, Vol 121, 3, June 2002, 333-355.

[K-K-K-W] R.Kadobianski, J.Kubarski, V.Kushnirevitch, and R.Wolak, Transitive Lie algebroids of rank 1 and locally conformal symplectic structures, Journal of Geometry and Physics 46 (2003) 151-158.

[K-M] J.Kubarski, A.S.Mishchenko, Lie algebroids: spectral sequences and signature, Sbornik: Mathematics, Volume 194(2003), Number 7, Pages 1079-1103.

[M] K. C. H. Mackenzie, Lie Groupoids and Lie Algebroids in Differential Geometry, Cambridge University Press, 124, 1987.

[S] M.Spivak, A Comprehensive Introduction to Differential Geometry, Vol. I., 2nd ed., Publish or Perish, Inc., Berkeley, 1979.

[W] A.Weinstein, The modular automorphism group of a Poisson manifold, J. Geom. Phys. 23 (1997), 379-394.

Institute of Mathematics, Technical University of Łódź, Poland, 90-924 Łódź, al. Politechniki 11

E-mail address: kubarski@mail.p.lodz.pl

$U R L:$ http://im0.p.lodz.pl/〜kubarski

Moscow State University, Moscow, Russia

E-mail address: asmish@higeom.math.msu.su

$U R L:$ http://higeom.math.msu.su/asmish/indexold.html 